\title{
UAV-based multi-angular measurements for improved crop parameter retrieval
}

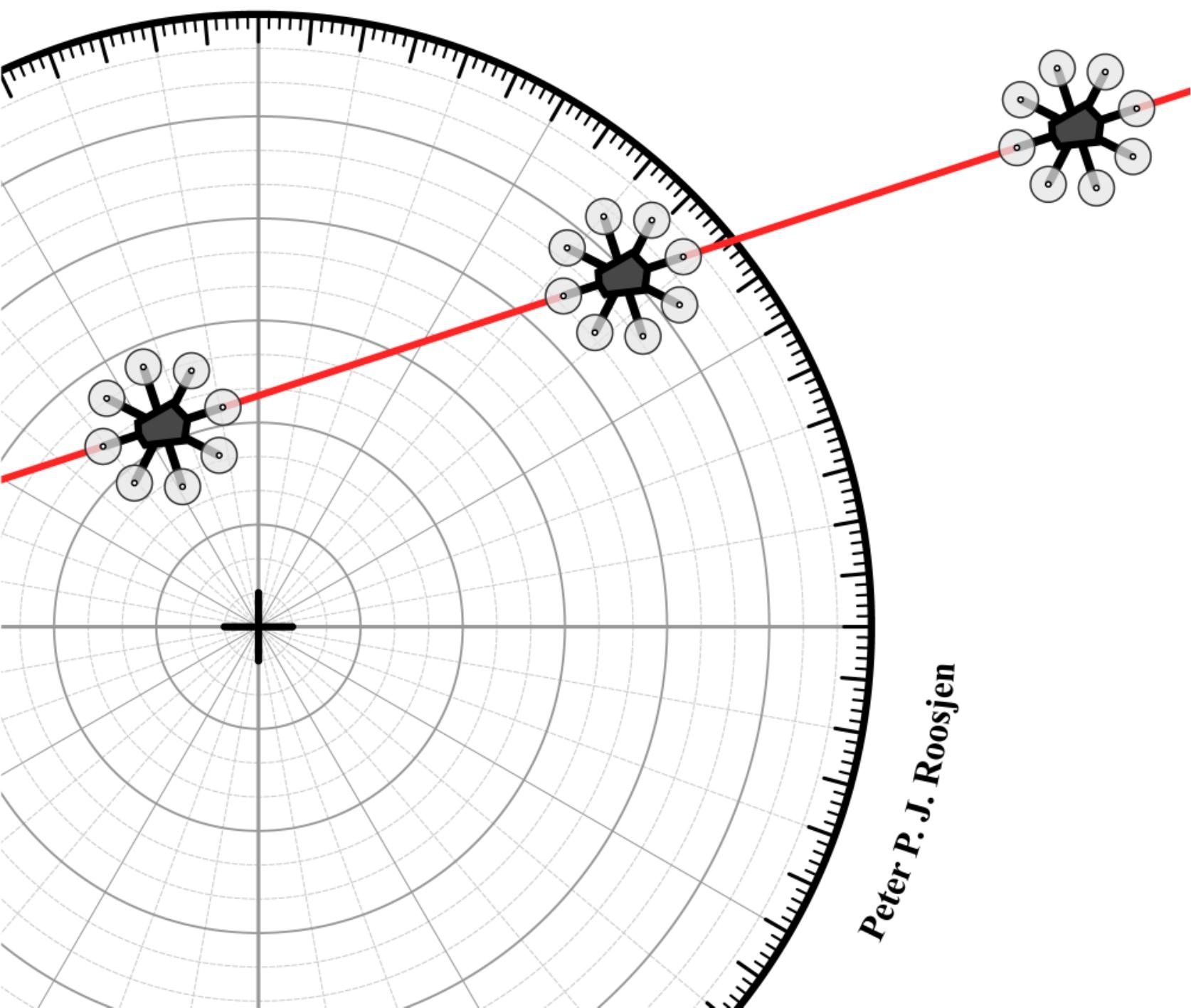




\section{Propositions}

1. Crop parameters can be better estimated using multi-angular observations.

(this thesis)

2. Unmanned aerial vehicles are the best tool for crop monitoring. (this thesis)

3. Effective science cannot be done on a single computer screen.

4. Basic programming should be part of high school education

5. Doing your work running leaves more time to enjoy life.

6. Contrary to what the word suggests, smartphones make people stupid.

Proposition belonging to the thesis entitled

"UAV-based multi-angular measurements for improved crop parameter retrieval"

Peter P. J. Roosjen

Wageningen, 17 November 2017 


\section{UAV-based multi-angular measurements for improved crop parameter retrieval}

Peter P. J. Roosjen 


\section{Thesis Committee}

\section{Promotor}

Prof. Dr M. Herold

Professor of Geo-information Science and Remote Sensing

Wageningen University \& Research

\section{Co-promotors}

Dr J.G.P.W. Clevers

Associate professor, Laboratory of Geo-information Science and Remote Sensing Wageningen University \& Research

Dr H.M. Bartholomeus,

Assistant professor, Laboratory of Geo-information Science and Remote Sensing Wageningen University \& Research

\section{Other members}

Prof. Dr E.J. van Henten, Wageningen University \& Research

Prof. Dr F.D. van der Meer, University of Twente, Enschede

Dr J. Verrelst, University of Valencia, Spain

Dr H. Aasen, ETH Zurich, Switzerland

This research was conducted under the auspices of the C.T. de Wit Graduate School of Production Ecology \& Resource Conservation (PE\&RC) 


\title{
UAV-based multi-angular measurements for improved crop parameter retrieval
}

\author{
Peter P. J. Roosjen
}

\section{Thesis}

submitted in fulfilment of the requirements for the degree of doctor at Wageningen University

by the authority of the Rector Magnificus,

Prof. Dr A.P.J. Mol, in the presence of the

Thesis Committee appointed by the Academic Board

to be defended in public

on Friday 17 November 2017

at 11 a.m. in the Aula. 
Peter P. J. Roosjen

UAV-based multi-angular measurements for improved crop parameter retrieval, 144 pages.

PhD thesis, Wageningen University, Wageningen, the Netherlands (2017) With references, with summary in English

ISBN 978-94-6343-671-7

DOI: $10.18174 / 421562$ 


\section{Contents}

\section{Page}

Chapter 1. Introduction 1

Chapter 2. Effects of soil moisture content on reflectance anisotropy-Laboratory goniometer measurements and RPV model inversions

Chapter 3. Hyperspectral reflectance anisotropy measurements using a pushbroom spectrometer on an unmanned aerial vehicle-Results for barley, winter wheat, and potato

Chapter 4. Mapping reflectance anisotropy of a potato canopy using aerial images acquired with an unmanned aerial vehicle

Chapter 5. Improved estimation of leaf area index and leaf chlorophyll content using multi-angle spectral data collected by an unmanned aerial vehicle

Chapter 6. Synthesis

References

Summary

Acknowledgements

List of publications

Short biography 



\section{Chapter 1}

Introduction 


\subsection{Introduction}

In the past decades, optical remote sensing has become a well-established technology for qualitative and quantitative characterization of the Earth's surface [1]. By providing spatial and temporal information in addition to spectral information, it allows for the study of surface parameters through space and time. In applications such as agriculture, it enables the monitoring of crop development during the growing season and allows, for example, the prediction of yield [2] or precision crop management [3]. Optical remote sensing techniques are particularly useful for providing timely, location-specific information on the biochemistry and structure of agricultural crops in order to help farmers optimize their management. However, this means that the provided information should be as accurate as possible.

Using optical remote sensing, biophysical crop parameters can be estimated based on the reflected solar light either through empirical-statistical methods, or through inversion of radiative transfer models (RTMs). However, natural surfaces reflect light anisotropically, which means that the light reflected by a surface varies with viewing and illumination geometry. As a consequence, objects may appear brighter or darker when viewed from different directions, resulting in different reflectance intensities at different viewing angles (figure 1.1), which may result in inaccuracies of the estimated parameters. In its idealized form, reflectance anisotropy is described by the bidirectional reflectance distribution function (BRDF), a function that quantifies the surface reflectance into a specific direction when being illuminated from a particular direction [4]. Besides viewing and illumination geometry, the magnitude of reflectance anisotropy is controlled by the optical characteristics of surface elements, such as leaves and soil facets, and their spatial and geometric arrangements [5]. For example, for a homogeneous vegetation cover, leaf orientation has a strong contribution to reflectance anisotropy $[6,7]$. For discontinuous vegetation, such as canopies with gaps or row-planted vegetation, the reflectance anisotropy is mainly driven by the proportion of visible of shadowed and sunlit vegetation and soil background, which can both contribute to the observed reflectance at particular observation geometries. Moreover, reflectance anisotropy also varies with wavelength.

For remote sensing purposes, the importance of reflectance anisotropy can be divided into two distinctive directions [8]. On the one hand, reflectance anisotropy can be seen as an unwanted effect that needs to be corrected for [9-12]. Correcting for anisotropy effects in remote sensing data, i.e., the transformation of data captured at a particular viewing and illumination geometry to a standardized viewing and illumination geometry, is necessary to make data collected at different times of the day (e.g., under different illumination angles due to movement of the sun) and/or data captured at different view angles (e.g., due to sensor orientation, differences in overpass etc.) comparable. On the other hand, reflectance anisotropy can be considered as an additional source of information to spectral data due to its unique response to surface structure $[13,5,14]$. Either way, knowledge on the magnitude of anisotropy effects is important. In this thesis, the main focus is on the 
extraction of additional information from reflectance anisotropy, with special attention to the biophysical properties of agricultural crops.
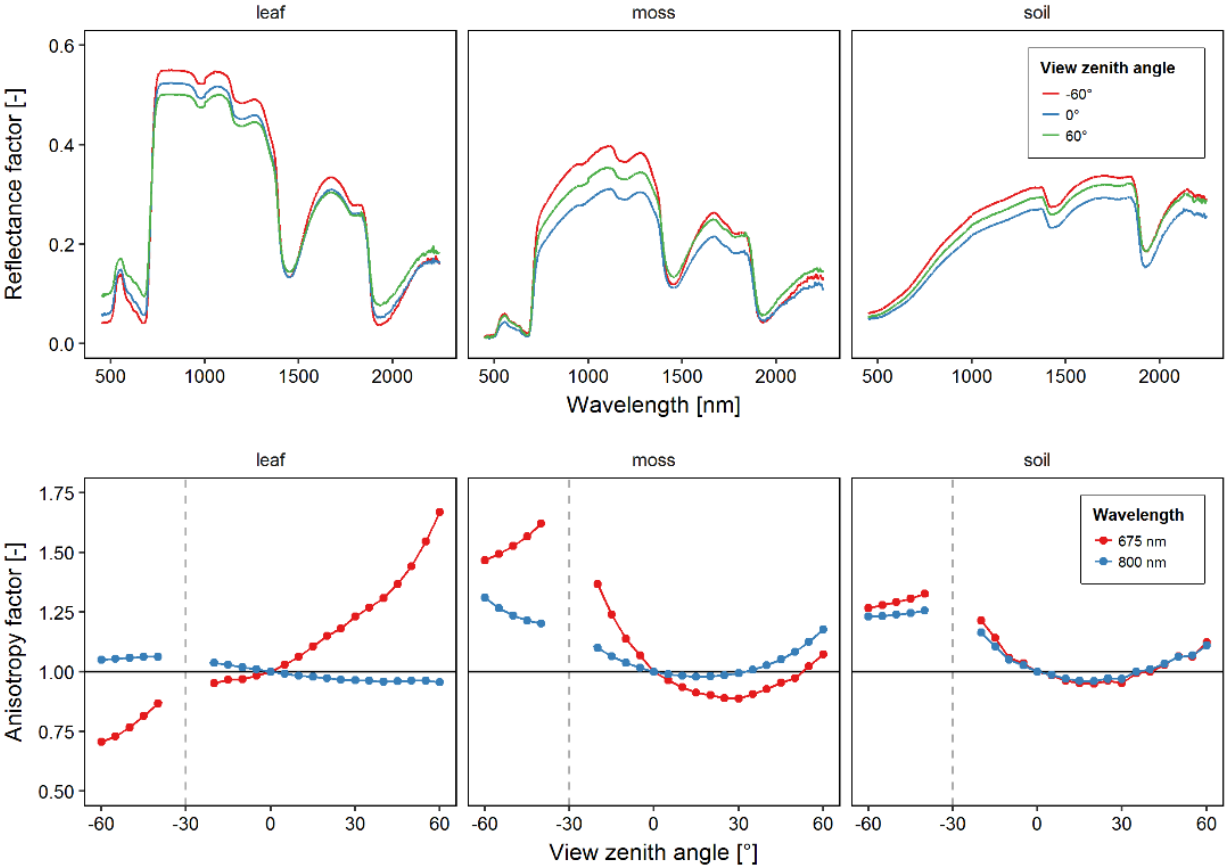

Figure 1.1. Spectral reflectance (top) and reflectance anisotropy (bottom), here depicted as the anisotropy factor, i.e., the nadir normalized reflectance, measured in the principal plane using a laboratory goniometer [15]. The illumination angle was set at $-30^{\circ}$ (i.e. the hotspot position), indicated by the vertical dashed-line. These measurements illustrate how reflectance factors change as a function of view zenith angle (VZA) in the principal plane at different wavelengths for a horizontally flat leaf (left), a moss sample (middle), and a soil sample (right). The flat leaf shows a clear increased reflectance in the forward scattering direction (positive VZAs) at $675 \mathrm{~nm}$, due to the glossy layer of the leaf, which results in mirror-like reflectance. On the contrary, the moss sample showed stronger backward scattering (negative VZAs) at $675 \mathrm{~nm}$, since in this direction the sensor was viewing the illuminated side of the moss sample. In contrast, the lowest reflectance factors were observed at a $30^{\circ} \mathrm{VZA}$, where the sensor was viewing the shaded side of the moss sample. At $800 \mathrm{~nm}$, the anisotropy effects were less pronounced, since shadows are less pronounced at this wavelength. The soil sample also showed increased backward scattering with only small differences at the displayed wavelengths. 


\subsection{Multi-angular measurements}

\subsubsection{Space-borne multi-angular measurements}

Reflectance anisotropy can be studied by performing multi-angular reflectance measurements. Some satellite-borne sensors, such as the Multi-angle Imaging Spectrometer (MISR) [16] or the Compact High Resolution Imaging Spectrometer on-board the Project for On-Board Autonomy (CHRIS-PROBA) [17], have been specifically designed to perform multi-angular observations. These sensors are capable of viewing an area of interest from several different angles during their overpass. The MISR has, in addition to a nadir-viewing camera, four pairs of fore and backward looking cameras that are able to capture a scene from in total nine different view angles during its overpass. The PROBA platform carrying the CHRIS sensor can be tilted during its overpass and is thereby able to collect multi-angular views of a scene. Instruments with a wide field of view (FOV), such as the Moderate Resolution Imaging Spectroradiometer (MODIS), are able to make multi-angular measurements of a surface based on the overlap of swaths during several overpasses. Whereas the CHRIS and MISR take several minutes to collect multiangular views, MODIS collects different views of a specific surface on different days. In areas of rapid change, such as the case of agricultural crops which change daily due to crop growth and development, the variation in observed reflectance might therefore not be the result of a difference in view angle, but rather an actual physical change of the observed area.

Space-borne multi-angular measurements typically lack either spatial, temporal, spectral, and/or angular resolution [18]. Especially, the relatively coarse spatial resolution of satellite data hinders detailed analysis for, e.g., application of precision agriculture, where a high spatial resolution is needed to capture within field variation of the grown crop [19].

\subsubsection{Ground-based multi-angular measurements}

Multi-angular measurements at a very high spectral and angular resolution are typically performed with goniometer systems. Ground-based goniometer systems allow for views of a target area throughout a measurement series, by positioning a sensor at different angles towards this area. In figure $1.2 \mathrm{a}$ a schematic representation of the measurement principal is displayed. Over the past decades, various goniometers that apply this measurement principal have been developed to perform multi-angular measurements under controlled laboratory conditions $[20,21,15,7]$ or under field conditions [22-27].

As a different measurement principle, a sensor can be rotated at a stationary position above a surface while viewing in all directions away from this position (figure 1.2b). This measurement principal is applied at, for example, the Automated Multiangular Spectro-radiometer for Estimation of Canopy reflectance (AMSPEC) [28-30] or the Portable Apparatus for Rapid Acquisition of the Observation of the Land and Atmosphere III (PARABOLA III) [31]. Using such an approach it is assumed that the observed surface is homogeneous within the FOV of the sensor. 

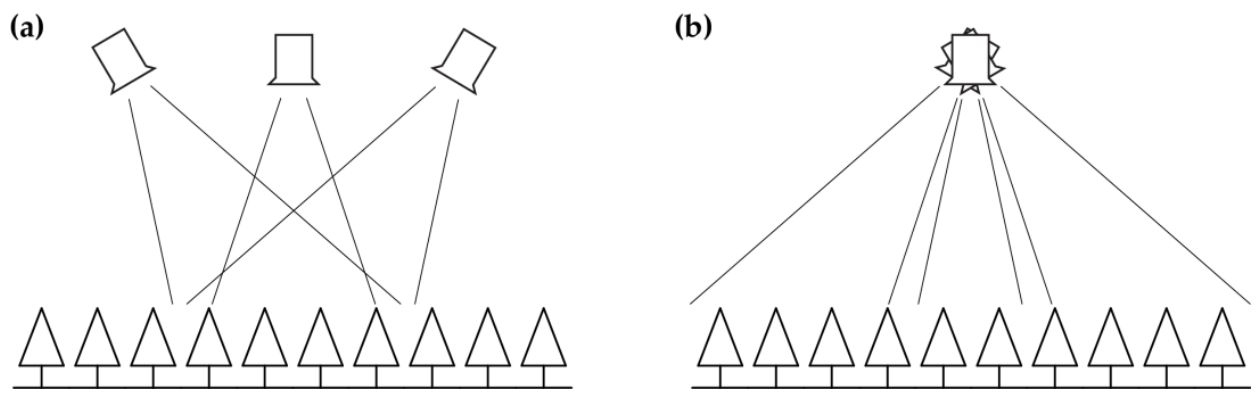

Figure 1.2.Schematic representation of two multi-angular measurements principals, based on [32].The sensor views the same spot from different directions (a); the sensor looks away from a stationary position and observes different parts of the surface (b), which are assumed to be homogeneous.

Although high quality multi-angular measurements can be obtained with goniometers [33], measurements by goniometers are typically limited to small surface areas with a relatively low three-dimensional structure, such as soils [34] or low vegetation, like grass [7]. The relatively short distance between target and sensor makes it difficult to capture a large enough representative area of natural heterogeneous targets, thereby making most goniometer setups unsuitable for multi-angular measurements of crops.

\subsubsection{UAV-based multi-angular measurements}

During recent years, UAVs rapidly have evolved into a robust and commonly used tool for remote sensing research [35]. The relatively low flight height of UAVs enables the collection of imagery at a high spatial resolution, which is needed for applications such as in precision agriculture [19]. Recently, several studies have explored the use of UAVs to collect multi-angular reflectance measurement [36-38]. As opposed to measurements using traditional ground-based goniometers, UAVbased multi-angular measurements do not have a ground base and do not depend on a moving arm to position a sensor at a specific view angle. This has several advantages:

- The studied surface does not get disturbed. Field goniometers such as, e.g., the field goniometer system (FIGOS) [39], leave large footprints caused by a circular rail, which forms the ground base of this system.

- Locations that are difficult to access can more easily be sampled with a UAV-based system, since the operator of the UAV does not need to physically enter the area of investigation. For example, when measurements are performed over an agricultural field, the operator can control (take off, fly, and land) the UAV from outside the field. Moreover, since UAV platforms are easily manoeuvrable, it is easier to sample several locations in a field during one, or multiple flights. On the other hand, ground-based goniometers typically only sample a single point. 
- $\quad$ The biggest limiting factor of ground-based goniometers for multi-angular measurements of crops is the limited distance between the observed target and the sensor, which makes it difficult, or even impossible to capture a surface covered by objects with a strong three-dimensional structure in the field of view (FOV) of the used sensor. The distance between the sensor and the target for UAVs can theoretically be as large as the technical specifications and the local legislation allow the UAV to fly.

Advantages of UAV-based multi-angular measurements over satellite-based measurements are the flexibility to perform the measurements when, and whenever needed (that is, if weather conditions and local legislations allow it). Moreover, the fine spatial resolution of UAV-based multi-angular measurements allows for analysis at a fine spatial scale, which is useful to study the relation between vegetation and reflectance anisotropy for applications such a precision agriculture. Until now, however, only a limited number of attempts to collect multiangular measurements using UAVs exist. On the one hand technical aspects of using UAVs for obtaining spectral measurements over a large range of different view angles still needs considerable research. An important issue is combining multiple views into a multi-angular signature at the pixel level. The second major problem is the translation of such UAV-based multi-angular observations into agricultural crop properties.

\subsection{Objectives}

The main objective of this thesis is to study the information content of multiangular measurements for deriving properties of natural surfaces, such as soil and vegetation, with a main focus on agricultural crops. In order to study the information content contained in the reflectance anisotropy signal, it is important to understand how reflectance anisotropy is influenced by characteristics of the observed target, which can be done by multi-angular measurements.

An important parameter for agricultural applications is soil moisture content (SMC), since it is an indicator of the water availability for crops. SMC is highly variable in space and time, making remote sensing an important tool to monitor SMC [40]. Traditional optical remote sensing methods for monitoring soil moisture are built upon the relation between reflectance and soil moisture based on empirically or physically based models and have been widely studied. The effects of SMC on the anisotropic reflectance behaviour of soils, however, did not receive much attention so far and are not well known [41-43]. Therefore, this results in the following research question:

\section{What is the effect of soil moisture content on reflectance anisotropy?}

Whereas satellites and goniometers have been typically used to perform multiangular measurements, satellites do not provide the spatial resolution needed for precision agricultural applications and goniometers are not suitable to perform 
measurements of surfaces with a strong three-dimensional structure, such as crops. The relatively low flight height of UAVs allows for the collection of imagery at a high spatial resolution, which is needed for applications such as precision agriculture [19], making them an interesting alternative for satellite-based measurements. Moreover, compared to ground-based goniometers, UAVs allow to take measurements with a larger sensor-target distance, thereby enabling to take directional measurements of crops. Therefore, a second objective was to explore the use of UAVs to perform multi-angular measurements. To explore the possibilities of UAVs for the study of reflectance anisotropy from a technical perspective, this results in the following research question:

\section{How can UAVs be used to study reflectance anisotropy?}

Agricultural management practices, such as irrigation and fertilization, are typically applied during specific development stages of crops. Spectral changes due to crop development have been widely studied. The effects of crop development on reflectance anisotropy, however, have not received much attention so far. This is addressed by the following research question:

\section{What is the effect of canopy development on reflectance anisotropy?}

Reflectance anisotropy can either be considered as a source of error, or as a source of additional information [8]. In this thesis, the main focus was to explore the reflectance anisotropy signal as a source of information, with a specific focus on crop parameters. This results in the following research question:

\section{How can reflectance anisotropy be used to improve crop parameter retrieval?}

\subsection{Thesis outline}

The chapters of this thesis are based on four peer-reviewed publications, each addressing one or more research questions as provided in section 1.3. Table 1.1 shows in which chapter each research question is addressed.

Table 1.1. Research questions and chapters.

\begin{tabular}{cc}
\hline Research question & Chapter \\
\hline 1 & 2 \\
2 & 3,4 \\
3 & 3,4 \\
4 & 5 \\
\hline
\end{tabular}

Chapter 2 examines the effects of SMC on reflectance anisotropy. In this chapter, we used a laboratory goniometer setup to perform multi-angular reflectance measurements of several soil samples in with different SMC levels. The effects of 
changes in the SMC level on the anisotropic reflectance behaviour of the soil samples were parameterized and quantified using a BRDF model.

Chapter 3 explores the possibility of performing multi-angular reflectance measurements with a hyperspectral pushbroom sensor mounted on a UAV. For this chapter, we took measurements of barley, potato and winter wheat at different growing stages and investigated the effects of canopy development of these crops on reflectance anisotropy.

Chapter 4 describes the use of a UAV to perform multi-angular measurements with a snapshot camera. Measurements were taken over an experimental potato field, where the potato plants in different zones in the field showed variation in development due to different applied fertilization regimes. In addition to capturing reflectance anisotropy with this system, we studied the variation of reflectance anisotropy due to differences in canopy development within the field.

Chapter 5 investigates the added value of multi-angular observations for improved crop parameter retrieval. The multi-angular measurements taken in chapter 4 were used to numerically invert the PROSAIL model to estimate leaf area index and leaf chlorophyll content. Moreover, we tested the performance of datasets simulated by the PROSAIL model at several multi-angular observation geometries for improved estimation of these parameters.

Chapter 6 summarizes the main outcomes of this this thesis in relation to the research questions. Furthermore, this chapter discusses the implications of the results and provides an outlook for future research avenues. 




\section{Chapter 2}

\section{Effects of soil moisture content on reflectance anisotropy-Laboratory goniometer measurements and RPV model inversions}

This chapter is based on:

Roosjen, P.P.J.; Bartholomeus, H.M.; Clevers, J.G.P.W. (2015). Effects of soil moisture content on reflectance anisotropy-Laboratory goniometer measurements and RPV model inversions. Remote Sensing of Environment, 170, 229-238. 


\begin{abstract}
Optical methods to study soil moisture content (SMC) are often based on empirically or physically based models that relate changes in reflectance intensity to SMC. The effects of SMC on the reflectance anisotropy, however, have not received much attention. In this paper the effects of SMC on the anisotropic reflectance behavior of soils were studied. Biconical reflectance factors (BCRFs) of five different soil samples were acquired at 60 positions covering the full hemisphere in the optical domain at different SMC levels using Wageningen University's laboratory goniometer facility. In addition, we inverted the RahmanPinty-Verstraete (RPV) model against the measured BCRFs in the principal plane. The results show that the anisotropic reflectance behavior of soils is strongly influenced by the SMC. Dry soils displayed strong backward scattering behavior, with a maximum reflectance close to the hotspot position. An increase of the SMC level up to the soil's saturation point caused the soils to scatter more in the forward direction and induced a weakening of the hotspot effect. Oversaturated soils displayed a strong sun-glint-like reflectance peak in the anti-solar direction. The RPV model fitted the measured BCRFs in the principal plane up to saturated SMC levels in general with an $\mathrm{R}^{2}>0.9$. It was not possible to fit the model through observations of oversaturated soils, since the RPV model is not capable of simulating specular reflectance. The asymmetry parameter $(\Theta)$ of the RPV model, which controls the proportion of forward and backward scattering, showed a strong correlation to SMC for individual samples. This correlation remained significant when we considered all samples together with a maximum $\mathrm{R}^{2}$ of 0.797 at $2123 \mathrm{~nm}$, indicating that reflectance anisotropy contained information on the water content of soils. The amplitude parameter $\left(\rho_{0}\right)$, which is closely related to the reflectance intensity, only had a maximum $\mathrm{R}^{2}$ of 0.622 at $1921 \mathrm{~nm}$. This indicated that reflectance anisotropy contained more information on SMC than the level of spectral reflectance as such.
\end{abstract}




\subsection{Introduction}

Soil moisture is an important parameter for environmental, ecological and agricultural processes. Knowledge about the distribution of soil moisture at high temporal and spatial scales is therefore of interest for many applications. Optical, thermal infrared and microwave remote sensing methods can provide this information about soil moisture [40]. Although thermal and microwave remote sensing offer well established methods for soil moisture estimation, the greater temporal availability and generally higher spatial resolution of optical remote sensing data make optical methods interesting for this purpose $[44,40]$. Optical methods are built upon the relation between reflectance and soil moisture and have been studied intensively. One of the earliest studies on this topic was carried out by Ångström [45], who investigated the fact that soils appear darker when they are wet. Ångström [45] assigned this darkening of soils upon wetting to an increase of internal reflections within the water layer that covers the soil particles. Internal reflections within this layer increase the amount of interactions between light and soil particles, which in turn increases the probability of light to be absorbed. Twomey et al. [46] and Lekner and Dorf [47] expanded on this theory by taking the different refractive indices $(\mathrm{n})$ of air $(\mathrm{n} \approx 1)$, soil $(\mathrm{n} \approx 1.5)$ and water $(\mathrm{n} \approx 1.33)$ into account. The decrease of the relative refractive index when light transfers from air to soil when soils are dry, and from air to water when soils are wetted increases the probability of light to penetrate the soil and thereby increases the probability of light to be absorbed by the soil particles. In addition to this, the absorption of light by free water within the soil matrix or water bound to soil particles plays an important role in changes in soil reflectance due to moisture [48]. Besides soil moisture, soil reflectance is influenced by intrinsic soil characteristics like soil composition (e.g. organic matter content, mineral composition), soil surface roughness (e.g. particle size distribution, aggregation, crusting) and soil color $[49,50]$. In general, an increase in soil surface roughness, soil organic matter content, soil moisture or interactions between them, results in a decrease of reflectance [51,41], which makes for example deriving SMC based on reflectance values alone a difficult task [52].

Several experimental studies into the effects of soil moisture on reflectance have been carried out. Lobell and Asner [42] measured the reflectance of several soil samples in the 350-2500 $\mathrm{nm}$ region at different SMC levels under laboratory conditions and found an exponential decrease in reflectance when SMC increased. Lobell and Asner [42] also found that reflectance in the visible and near-infrared (VNIR) saturated at lower SMC levels compared to reflectance in the shortwave infrared (SWIR). Weidong et al. [53] performed a similar experiment and found that the decrease in reflectance is only valid up to a soil specific SMC level, which they referred to as the critical point. After the critical point, which was correlated with the point of near-saturation $(-0.01 \mathrm{MPa}$ water potential) of a soil, the soil reflectance increases with increasing soil moisture.

A lot of effort has been invested into understanding, measuring [42,53] and modelling $[52,54,55,43,56]$ the effects of SMC on soil reflectance and predicting SMC based on reflectance data $[57,58]$. Although there is a clear overview on the 
effects of SMC on spectral changes in reflectance, the effects of SMC on the anisotropic reflectance behavior of soils did not receive much attention so far [4143]. Multi-angular reflectance measurements are able to provide a more complete insight on the soil surface composition and structure $[59,60]$, and possibly also in SMC. Recently, Rosendahl et al. [61] and Croft et al. [41] studied changes in reflectance of soils at different SMC levels, observed from different observation positions. Rosendahl et al. [61] measured the reflectance of sand grains and glass spheres at different SMC levels from several positions in the principal plane and observed for both materials a relatively higher reflectance intensity in the forward scattering direction. Croft et al. [41] also studied the changes in reflectance due to soil moisture in the principal plane and found a stronger relation between the reflectance measured from forward scattering positions and soil moisture, compared to other observation positions. However, the soil samples used in the study of Croft et al. not only varied in SMC level, but also in soil surface roughness and soil organic carbon, which made it impossible to assign changes in observed reflectance at different viewing angles solely to soil moisture.

This study aims to further explore the multi-angular reflective domain in relation to SMC by measuring and modelling the effects of SMC on reflectance anisotropy under controlled conditions. The number of attempts in quantitatively modelling the reflectance anisotropy of soils is limited. Physical approaches are based on the soil model developed by Hapke [62] for describing the radiometric properties of planetary surfaces. This was validated by Pinty et al. [63] for terrestrial soil surfaces. Subsequently, Jacquemoud et al. [64] extended the model to include both backward and forward scattering for smooth soils. However, such a physically based model requires a rather large number of parameters, which may inhibit an accurate model inversion against real measurements. Therefore, a semi-empirical approach is followed in this study. Multi-angular reflectance measurements in the optical domain of five different soil samples with varying SMC levels were acquired with a laboratory goniometer setup [15]. The semi-empirical Rahman-PintyVerstraete (RPV) model [63] was inverted against these measurements to parameterize the measured anisotropy patterns at different SMC levels. The RPV model is of particular interest since it simulates surface anisotropy on the basis of only four parameters. The parameters obtained by the inversion of the RPV model were used to study the relation between reflectance anisotropy patterns and SMC.

\subsection{Methods}

\subsubsection{Soil samples}

Five soil samples, collected at different locations in the Dutch provinces of Friesland $\left(53.13^{\circ} \mathrm{N}, 5.82^{\circ} \mathrm{E}\right)$ and Noord-Brabant $\left(51.67^{\circ} \mathrm{N}, 5.00^{\circ} \mathrm{E}\right)$ in 2009 and 2010, respectively, were used in this experiment (table 2.1). For the experiments, the samples were placed in a mat-black coated cylindrical sample holder made of PVC, with a $20 \mathrm{~cm}$ diameter and a $0.1 \mathrm{~cm}$ depth. The sample holders were filled to the brim with soil and flattened. Deionized water was added to the soil until an 
oversaturated state was reached. The samples were left to rest for $30 \mathrm{~min}$ so that the water could distribute uniformly along the sample. Subsequently, any excess water after this point was drained from the surface.

The volumetric soil moisture content $(\theta)$, hereafter referred to as SMC, of the samples was calculated based on the oven-dry $\left(24 \mathrm{~h}\right.$ at $\left.105{ }^{\circ} \mathrm{C}\right)$ weight of the samples $\left(m_{0}\right)$ and the weights of the sample collected during the drying process $(m)$, using equation 2.1 .

$$
\theta=\frac{\left(m-m_{0}\right) / \rho_{w}}{m_{0} / \rho_{b}}
$$

where $\rho_{w}$ is the water density $\left(0.998 \mathrm{~g} \cdot \mathrm{cm}^{-3}\right.$ at $\left.23{ }^{\circ} \mathrm{C}\right)$ and $\rho_{b}$ is the bulk density of the soil sample (table 2.1). The weights of the samples were recorded during the experiment at a one minute time interval with a logged digital balance with one milligram accuracy. Each soil was measured until stable weight was reached and thus all the water was evaporated. The weights of the samples were synchronized to the time point of the spectral measurements (see section 2.2.2) by linear interpolation. The soils were visually checked for changes in roughness after adding the water and after the drying process. We did not observe any changes in roughness in the samples, aside from sample s10-081-12 which slightly swelled up after adding water. However, the sample did not shrink during the drying process. Therefore, we made the assumption that the soil roughness remained unchanged during the experiment and all observed effects were caused by changes in SMC level.

In addition to SMC, we also expressed the amount of water in the soil as the relative water content (RWC) [65], since each soil type can only hold a specific absolute amount of water. The RWC can be considered as the fraction of saturation of a soil and is calculated by equation 2.2 .

$$
R W C=\frac{\theta}{\theta_{\text {Sat }}}
$$

where $\theta_{\text {Sat }}$ is the soil specific saturation point (table 2.1). RWC $=0$ corresponds to an oven-dry soil and RWC $=1$ corresponds to a saturated soil.

\subsubsection{Multi-angular reflectance measurements}

The anisotropic reflectance behavior of the samples was measured with Wageningen University's laboratory goniometer setup [15]. The system is schematically illustrated in figure 2.1. The soil samples were illuminated by a Quartz Tungsten Halogen (QTH) lamp, set at $900 \mathrm{~W}$, which was placed $70 \mathrm{~cm}$ from the sample at a $30^{\circ}$ zenith angle. Multi-angular reflectance measurements of the samples were taken in the 350-2500 nm region with an ASD FieldSpec 3 spectrometer (Analytical Spectral Devices, Boulder, CO), while they were left to dry out. The spectrometer was equipped with a diffuser (ASD Scrambler) and a $1^{\circ}$ fore 
optic, which resulted in a measured area on the ground of $3.8 \mathrm{~cm}^{2}$ at nadir position and of $9.1 \mathrm{~cm}^{2}$ at a view zenith angle (VZA) of $65^{\circ}$, which was the largest angle used in this study. A white Spectralon panel (LabSphere Inc., North Sutton, $\mathrm{NH}$ ) was used to calibrate the spectrometer. The goniometer setup was built in a climate cell that was covered with highly absorbent black materials to exclude diffuse illumination. Anisotropic reflectance characteristics of a surface are often referred to as the bidirectional reflectance distribution function (BRDF), which can be approximated by bidirectional reflectance factors (BRFs) covering the full hemisphere. The BRF is a quantity defined as the ratio of radiance reflected from a surface to the radiance reflected from an ideal Lambertian surface for a given view and illumination geometry and wavelength. Due to the conical field of view and illumination of the spectrometer and lamp used in our measurements setup, the correct terminology for reflectance factors that were measured for this study is conical-conical reflectance factors (CCRFs) or biconical reflectance factors (BCRFs), following the nomenclature of Schaepman-Strub et al. [4]. Hereafter, we will refer to the measured reflectance factors as BCRFs. The BCRFs measured for this study are a good approximation of BRFs due to the narrow field of view $\left(1^{\circ}\right)$ of the spectrometer.

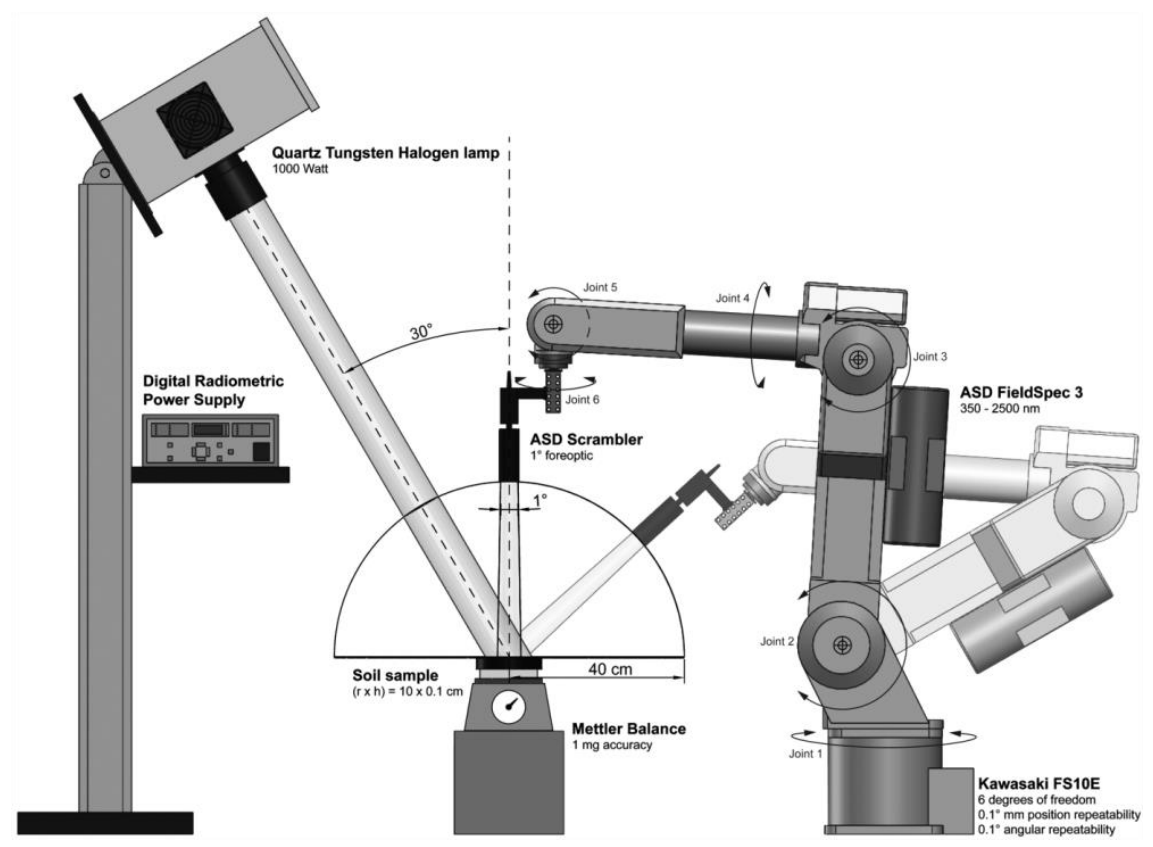

Figure 2.1. Schematic representation of the measurement setup. The robot arm is used to position the spectrometer at different positions around the soil sample. A fiber optic cable (not drawn) connects the fore optic to the spectrometer.

During the measurements, the temperature in the climate cell was maintained at 23 ${ }^{\circ} \mathrm{C}$ and the humidity at $50 \%$. The robot arm, which forms the core of the goniometer, was programmed to position the spectrometer at 60 different positions around the 
samples. Measurements were taken at an angular resolution of $30^{\circ}$ in the azimuth plane and at a resolution of $15^{\circ}$ in the zenith plane from $-60^{\circ}$ to $+60^{\circ}$. In the principal plane, where most of the variation in reflectance is expected, measurements were taken every $5^{\circ}$ zenith from $-65^{\circ}$ to $+65^{\circ}$. VZAs close to the hotspot $\left(-35^{\circ}\right.$ to $\left.-25^{\circ}\right)$ were programmed at a $15^{\circ}$ azimuth angle off the principal plane to avoid shadows of the robot arm or sensor on the target. A complete measurement cycle of 60 positions covering the full hemisphere took approximately $23 \mathrm{~min}$. The 60 positions were repetitively measured (9-18 times, depending on the sample, see table 2.1) until the soil reached a stable (air-dry) weight. Water was continuously evaporating from the samples during the dry-out experiment, which had as a consequence that the SMC level of each reflectance measurement in a measurement cycle was slightly different. To correct for the loss in soil moisture we applied a linear interpolation between the BCRFs at each measurement position of two consecutive SMC levels. In addition to correct for the loss in soil moisture, this interpolation enabled us to assign a single SMC level to all spectral measurements within a measurement cycle.

Table 2.1. Properties of the soil samples used in this study. The texture classes are within a $1 \%$ detection limit. Field capacity and wilting point were measured at $\mathrm{pF}=2.0$ and $\mathrm{pF}=4.2$, respectively.

\begin{tabular}{|c|c|c|c|c|c|c|c|c|c|c|}
\hline Sample & 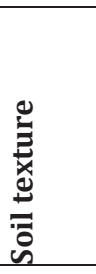 & 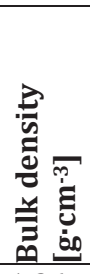 & $\begin{array}{l}\frac{a}{a} \\
\vdots \\
\frac{N}{3} \\
v\end{array}$ & 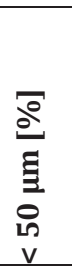 & $\begin{array}{c}\frac{0}{2} \\
\vdots \\
\vdots \\
0 \\
10 \\
1\end{array}$ & $\frac{e^{\circ}}{\overline{0}}$ & 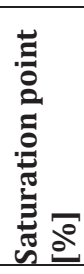 & 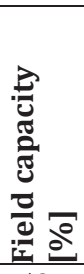 & 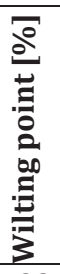 & 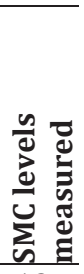 \\
\hline s09-131-34 & loam & 1.26 & 23.9 & 59.7 & 28.5 & 8.16 & 64 & 59 & 22 & 12 \\
\hline s10-050-30 & sand & 1.68 & 2.4 & 3.0 & 95.3 & 1.53 & 52 & 27 & 2 & 9 \\
\hline s10-081-12 & $\begin{array}{c}\text { sandy } \\
\text { loam }\end{array}$ & 1.14 & 2.2 & 6.7 & 72.9 & 23.0 & 63 & 26 & 13 & 18 \\
\hline s10-081-13 & sand & 1.77 & 1.9 & 1.3 & 97.6 & 1.2 & 47 & 18 & 3 & 9 \\
\hline s10-081-17 & loam & 1.29 & 9.4 & 14.6 & 52.8 & 2.87 & 59 & 50 & 11 & 16 \\
\hline
\end{tabular}

${ }^{*}$ Organic matter and calcium content based on Loss on Ignition (LOI) calculated as $100 *$ (weight sample at $105^{\circ} \mathrm{C}$ - weight sample at $550^{\circ} \mathrm{C}$ ) / weight sample at $105^{\circ} \mathrm{C}$.

\subsubsection{Rahman-Pinty-Verstraete model}

The Rahman-Pinty-Verstraete (RPV) model [66] was inverted against the goniometer measurements based on a lookup table (LUT) approach. The RPV model is an empirical model which gives a parametric representation of reflectance anisotropy based on (i) intensity of reflectance, (ii) bowl and bell-shaped anisotropy curvature, (iii) forward and backward scattering intensity, and (iv) hotspot effects. The RPV model simulates the directional reflectance of a surface into direction $\left(\theta_{r}, \varphi_{r}\right)$ illuminated from direction $\left(\theta_{i}, \varphi_{i}\right)$ using four parameters. The model splits the reflectance into a scalar component $\rho_{0}$, which is an arbitrary 
parameter that characterizes the surface reflectance intensity and a directional component (equation 2.3).

$$
\rho_{s}\left(\theta_{i}, \varphi_{i} ; \theta_{r}, \varphi_{r}\right)=\rho_{0} \frac{\cos ^{k-1} \theta_{i} \cos ^{k-1} \theta_{r}}{\left(\cos \theta_{i}+\cos \theta_{r}\right)^{1-k}} F(g)[R(G)]
$$

The Minnaert parameter $(k)$ controls the bell-shape $(k>1)$ and bowl-shape $(k<1)$ of the anisotropy curve. The Henyey-Greenstein phase function $F(g)$, [67] which is driven by the asymmetry parameter $(\Theta)$, controls the amount of forward $(\Theta>0)$ and backward scattering $(\Theta<0)$, as given in equation 2.4 .

$$
F(g)=\frac{1-\Theta^{2}}{\left[1+\Theta^{2}-2 \Theta \cos (\pi-g)\right]^{1.5}}
$$

where the phase angle $g$ is given by equation 2.5 .

$$
g=\cos \theta_{i} \cos \theta_{r}+\sin \theta_{i} \sin \theta_{r} \cos \left(\varphi_{i}-\varphi_{r}\right)
$$

The hotspot function $R(G)$, driven by the hotspot parameter $\left(\rho_{c}\right)$, is approximated by equation 2.6 .

$$
R(G)=1+\frac{1-\rho_{c}}{1+G}
$$

where the geometrical factor $G$ is defined by equation 2.7 .

$$
G=\left[\tan ^{2} \theta_{i}+\tan ^{2} \theta_{r}-2 \tan \theta_{i} \tan \theta_{r} \cos \left(\varphi_{i}-\varphi_{r}\right)\right]^{1 / 2}
$$

For the inversion of the RPV model, a lookup table was created for the reflectance factors at all measurement positions in the principal plane using the $\rho_{0}, k, \Theta$ and $\rho_{c}$ parameters as given in table 2.2 .

Table 2.2. Range and the step size of the RPV parameters as used to create the lookup table.

\begin{tabular}{cccc}
\hline Parameter & Minimum & Maximum & Step size \\
\hline$\rho_{0}$ & 0.0 & 1.0 & 0.01 \\
$k$ & 0.0 & 2.0 & 0.01 \\
$\Theta$ & -1.0 & 1.0 & 0.01 \\
$\rho_{c}$ & 0.0 & 1.0 & 0.01 \\
\hline
\end{tabular}

Reflectance simulations of the RPV model based on all combinations of the parameters in table 2.2 were fitted through the BCRFs that were measured in the principal plane with the goniometer. The combination of parameters that produced the best fit with the measurements was determined based on the lowest root mean square error (RMSE) between the measured $\left(R_{\text {Gonio }}\right)$ and modelled reflectance $\left(R_{R P V}\right)$ at all positions in the principal plane (equation 2.8). 


$$
R M S E=\sqrt{\frac{\sum_{i=1}^{n}\left(R_{\text {Gonio }}-R_{R P V}\right)^{2}}{n}}
$$

where $n$ is the number of measurement positions in the principal plane.

\subsection{Results}

In this section, we present the effects of SMC on reflectance anisotropy that were measured with the goniometer and simulated with the RPV model. Sample s09131-34 (table 2.1) is used for illustration purposes, because all investigated samples showed similar responses to changes in SMC level.

\subsubsection{Dry-out experiment}

The complete time line of the complete dry-out experiment of the sample s09-13134 is shown in figure 2.2. The SMC level gradually dropped from $88.41 \%$ in the beginning of the experiment until it reached a stable SMC level of 7.45\% in the end. The robot was programmed to repetitively measure the reflectance of the sample from 60 positions, covering the full hemisphere. In the case of sample s09-131-34, these measurements were repeated 12 times during the timespan of the dry-out experiment. For the other samples it took between 9 and 18 repetitions of the reflectance measurements at all programmed positions until a stable SMC level was reached (table 2.1, SMC levels measured).

The SMC level constantly decreased during the time span of the dry-out experiment. The decrease in SMC level during the time that it took to measure a complete hemisphere of 60 measurements was in some cases over $8 \%$. The maximum decrease in SMC level during the time that it took to measure all 24 positions in the principal plane was just over 2\% (figure 2.3). All BCRFs within a measurement cycle were transformed to the estimated BCRFs at the moment of the nadir measurement (section 2.3.3).

\subsubsection{Effects of SMC on reflectance}

Figure 2.4a shows the effect of SMC level on the spectra observed from nadir position. Over the measured spectral domain, the spectrum of the air-dry sample $(7.45 \%)$ had the highest reflectance values. Reflectance decreased with increasing SMC, except for oversaturated SMC levels $(>64 \%)$ in the VNIR region of the spectrum. Here, the reflectance values went up again. The strongest spectral variations were found in the SWIR region of the spectrum with emphasis on the water absorption bands centered at 1450 and $1900 \mathrm{~nm}$. In these regions, a change of the shape of the spectral curve was observed. Upon wetting, the shape of the spectral curve hardly changed in the visible region of the spectrum, which is related to darkening of the soil without any change in the color. 


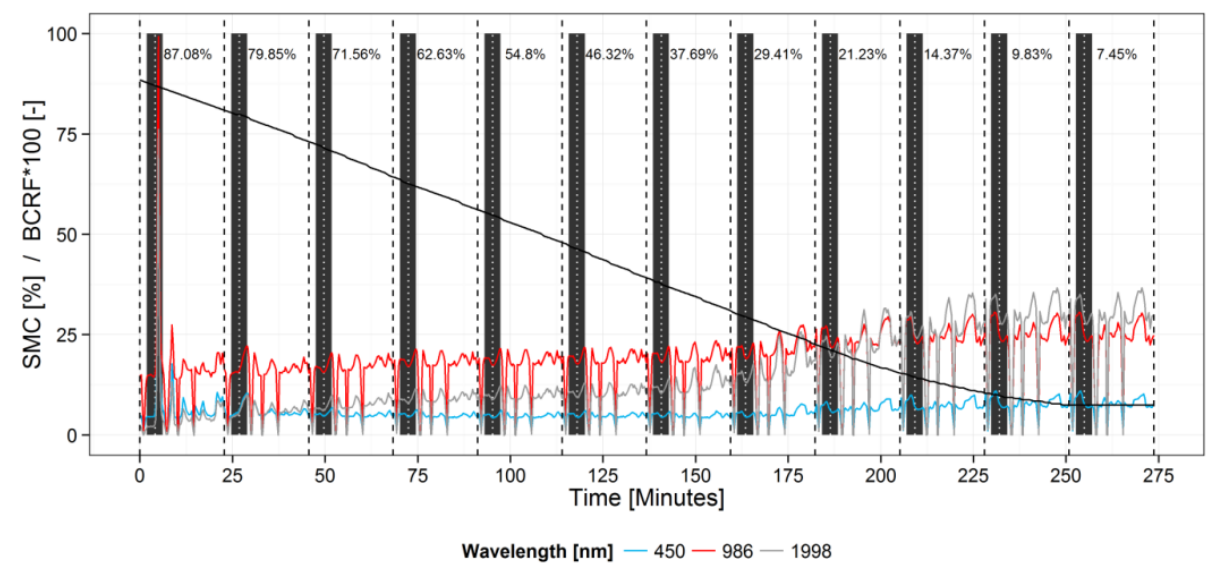

Figure 2.2. BCRFs (coloured lines) of sample s09-131-34 observed at all measurement positions covering the full hemisphere and SMC level (black line) over the time of the dry-out experiment. The percentages $(87.08 \%-7.45 \%)$ indicate the SMC level at the time that the nadir reflectance was measured (white dotted lines). The dark-grey bars indicate the measurements in the principal plane, where the BCRFs on the left-side of the nadir-line correspond to the backward scattering positions and the BCRFs on the right-side of the nadir-line correspond to the forward scattering positions. Per measured hemisphere there are several dips where the BCRFs dropped close to 0 . This is where the robot was at a position between the lamp and the sample and thus casting a shadow over the sample, resulting in a low measured signal.

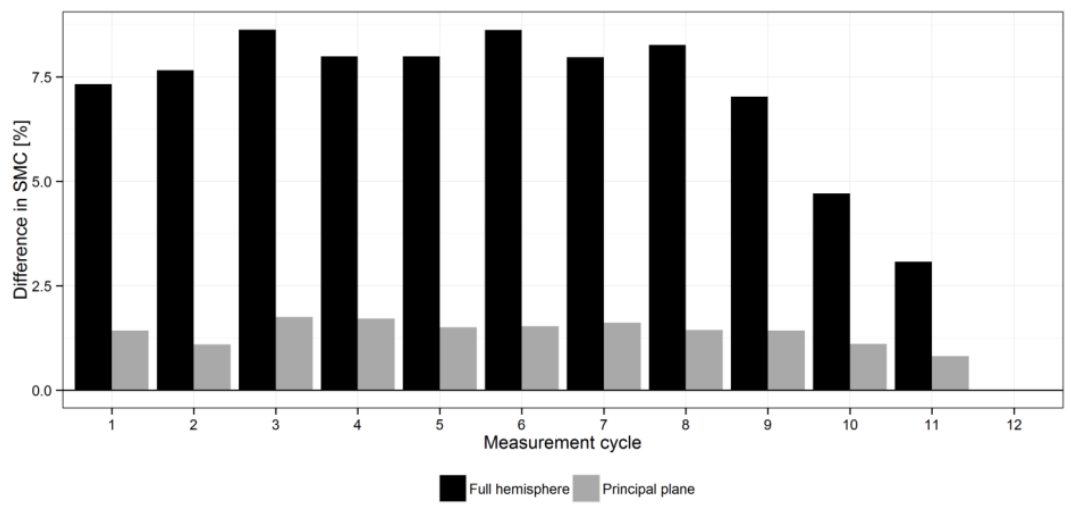

Figure 2.3. The absolute decrease in SMC level between the start and end of each measurement cycle over the full hemisphere (black bars) and the principal plane (grey bars) for sample s09-131-34. The SMC level stabilized at the end of the $11^{\text {th }}$ measurement cycle (figure 2.2 , at $\mathrm{t}=250 \mathrm{~min}$ ), resulting in no more changes in SMC level between the start and the end of the last $\left(12^{\text {th }}\right)$ measurement cycle.

An increasing SMC level caused a non-linear and wavelength dependent decrease of the BCRFs (figure 2.4b). Wavelengths in the visible region were only sensitive to an increase of SMC level from air-dry up to $30 \%$. After this point, the BCRFs did not 
decrease any further. Wavelengths in the NIR and SWIR region were sensitive to changes of SMC level within The whole range from air-dry to oversaturation. The BCRFs decreased the strongest between an air-dry state and an SMC level of $30 \%$. The reflectance decreased at a lower rate with increasing SMC levels between 30\% and the saturation point. A slightly faster rate of decrease in reflectance occurred with increasing SMC levels after the saturation point for wavelengths in the NIR and SWIR regions. The BCRFs at $450 \mathrm{~nm}$ and $574 \mathrm{~nm}$ increased again at SMC levels beyond the saturation point.
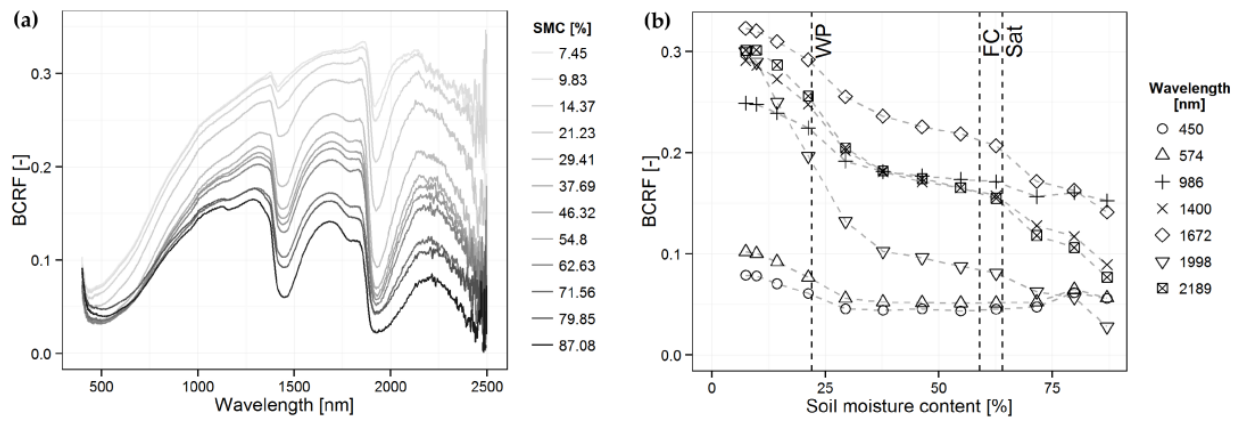

Figure 2.4. (a) Spectra of sample s09-131-34 observed from nadir position at SMC levels ranging from air-dry to oversaturation. (b) BCRFs at several wavelengths as function of SMC. WP = wilting point $(22 \%), \mathrm{FC}=$ field capacity $(59 \%)$ and Sat $=$ saturation point $(64 \%)$.

\subsubsection{Effects of SMC on reflectance anisotropy}

Due to the constant decrease in SMC level during the time span of the dry-out experiment (figure 2.2) it was not possible to link one SMC level to the measured reflectance values at all measurement positions of each cycle over the full hemisphere. To overcome this problem, we linearly interpolated the BCRFs per measurement position between two consecutive SMC levels. figure 2.5a shows the result of the interpolation for the BRCF measurements in the principal plane. Here, the BCRFs measured at all positions in the principal plane were interpolated to the SMC level at the time that the nadir position was measured. The differences in SMC of around $2 \%$ during the time of a principal plane scan (figure 2.3) showed to have an impact on the differences between measured and interpolated BRCFs (figure $2.5 b)$. Overall, these differences were small ( $<1 \%$ in the $71.56 \%-7.45 \%$ SMC range). At oversaturated SMC levels, a specular reflectance peak appeared. Here, the differences between measured and interpolated BCRFs were larger (up to 4\%) due to the fast emergence of this specular peak as the SMC level increased beyond the saturation point of the soil.

Figure 2.6 shows interpolated polar contour plots of the effects of SMC on the changes in BCRFs over the full hemisphere, illustrated for three different wavelengths. The highest BCRFs were observed in the backward scattering direction, with a maximum close to the angle of incidence at an air-dry state of the soil sample (7.45\%). A clear hotspot was visible at $450 \mathrm{~nm}$. We observed the lowest 
BCRFs in the forward scattering direction, where mostly the shaded sides of the soil particles were viewed by the spectrometer. A decreased proportion of backward scattering and an increased proportion of forward scattering were observed at an intermediate SMC level (37.69\%). Here, the hotspot of the air-dry sample was no longer visible. At an SMC level of 87.08\%, a sun-glint-like reflectance peak appeared with maximum BCRFs around the angle of specular reflectance $\left(\theta=30^{\circ}, \varphi=0^{\circ}\right)$.
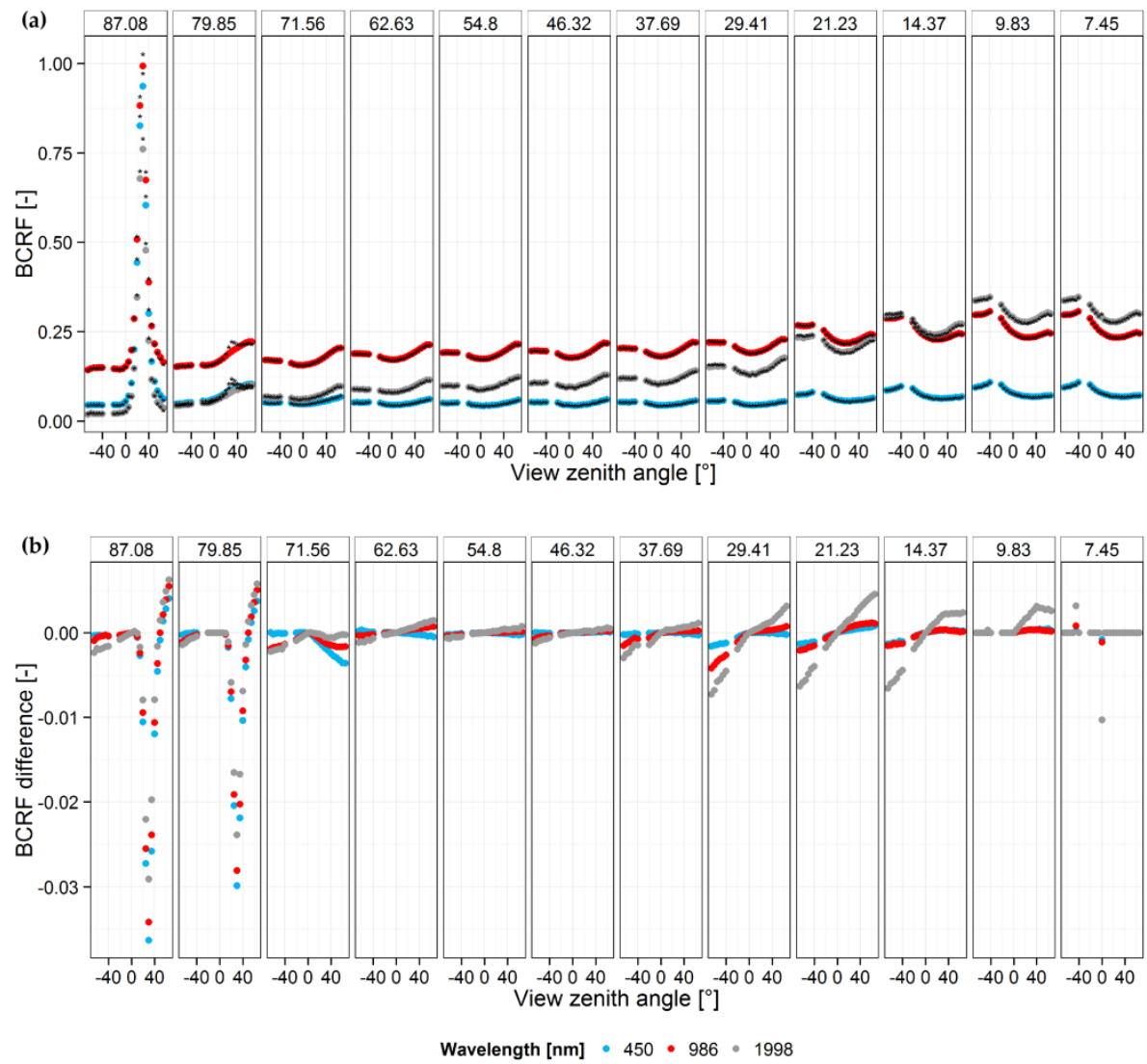

Figure 2.5. (a) Measured BCRFs at several different wavelengths (coloured dots) and their corresponding interpolated BCRFs (black dots). (b) Difference between measured and interpolated BCRFs. 

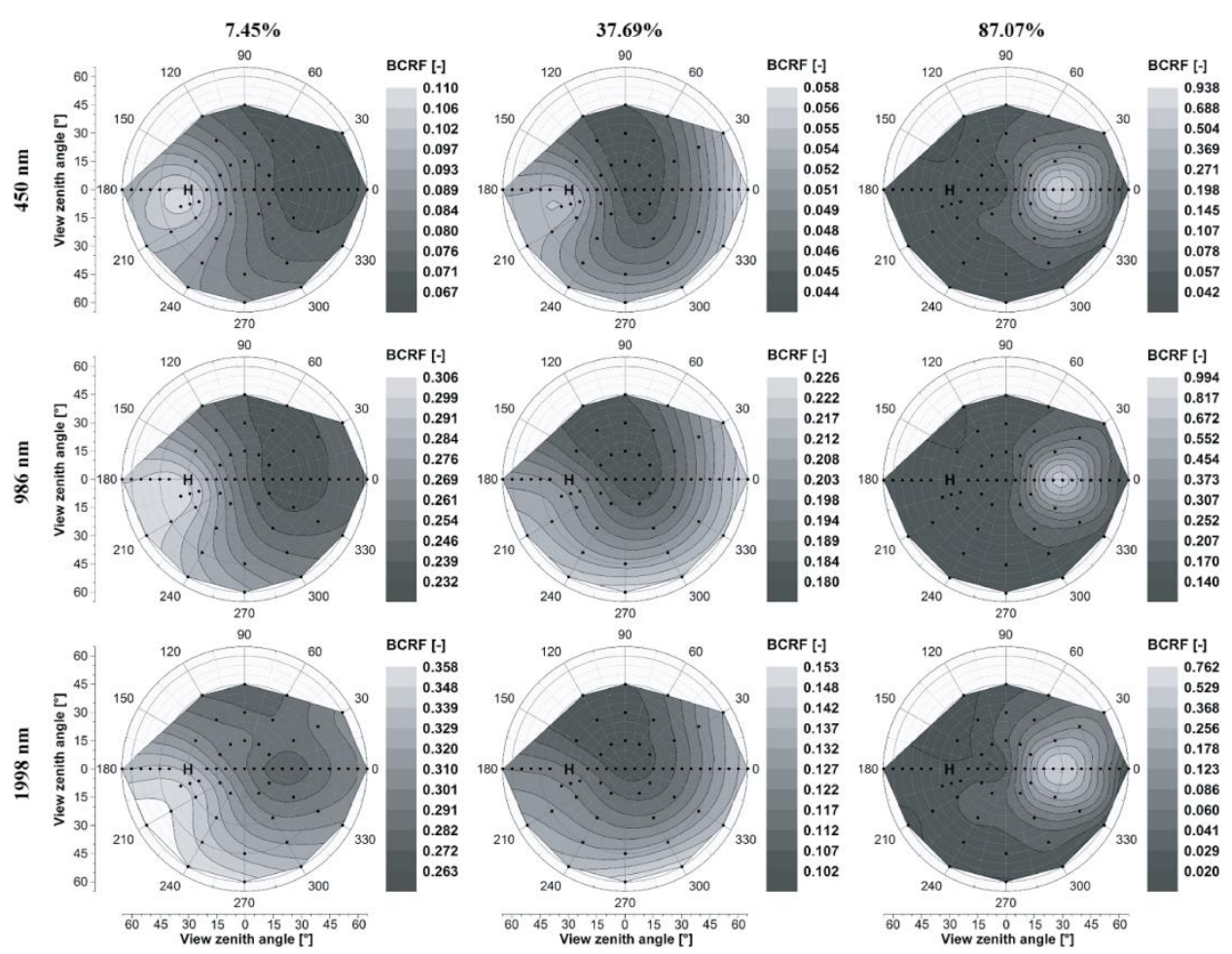

Figure 2.6. Changes in reflectance anisotropy due to SMC shown at 450, 986 and $1998 \mathrm{~nm}$ at an air-dry (7.45\%), intermediate (37.69\%) and oversaturated (87.08\%) state. The ' $\mathrm{H}$ ' indicates the hotspot position $\left(\theta=30^{\circ}, \varphi=180^{\circ}\right)$ and the black dots indicate the measurement positions. The BCRFs at $87.08 \%$ are plotted on a logarithmic scale to capture the sun glint effect.

\subsubsection{Modelling the effects of SMC on reflectance anisotropy}

The RPV model was used to create a LUT for the reflectance at all measurement positions in the principal plane based on all different combinations of the $\rho_{0}, k, \Theta$ and $\rho_{c}$ parameters (table 2.2). Each modelled reflectance value was compared to the BCRF measurements obtained with the goniometer. The combination of parameters that produced the lowest RMSE between measured and modelled reflectance factors in the principal plane between a VZA of $-65^{\circ}$ and $65^{\circ}$ was considered the parameter combination that best fitted the measurements. Figure 2.7a displays for the 400-2200 $\mathrm{nm}$ region the $\mathrm{R}^{2}$ between measured and modelled reflectance factors in the principal plane at all SMC levels based on the parameters obtained by inversion of the RPV model. The 350-400 nm and 2200-2500 nm regions were not inverted, due to the presence of noise at these wavelengths. The fit of the RPV model overall was accurate with $\mathrm{R}^{2}>0.9$. However, the model fit was less accurate in the 400-420 $\mathrm{nm}$ region. The reflectance measurements at these wavelengths were still slightly noisy which resulted in "bumpy" anisotropy curves through which it was not possible to fit the RPV model smoothly and therefore 
resulting in relatively lower $\mathrm{R}^{2} \mathrm{~s}$. Simulation of the reflectance factors over the full hemisphere based on the parameters obtained by inversion of the reflectance factors in the principal plane resulted in a less good fit as shown in figure $2.7 \mathrm{~b}$.

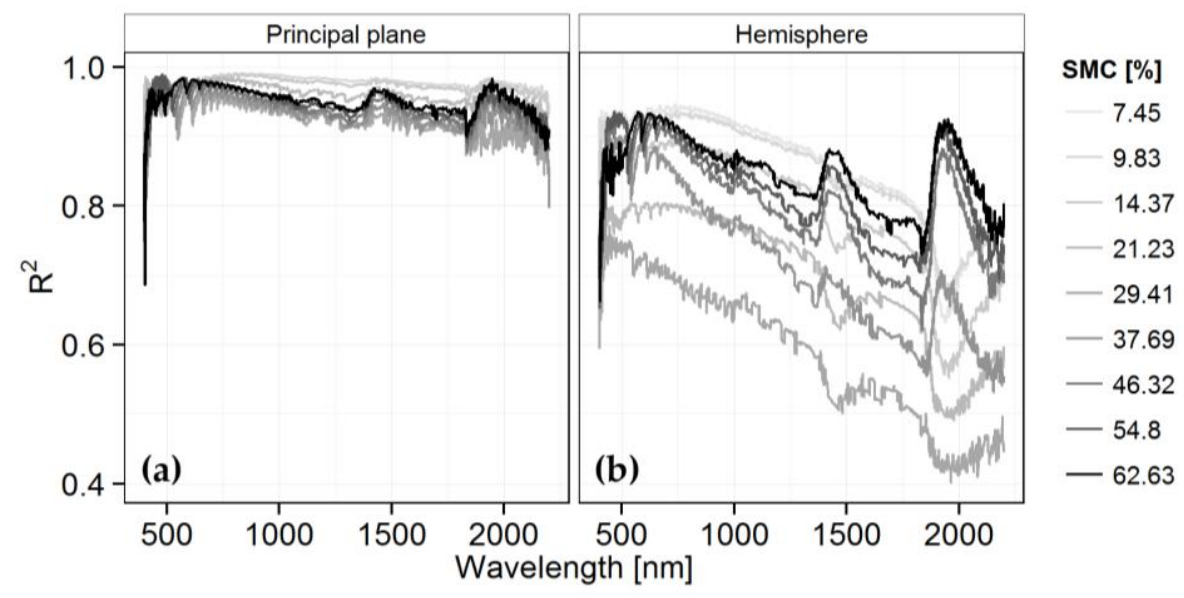

Figure 2.7. The $\mathrm{R}^{2}$ of fit of the RPV model based on the parameters obtained by inversion of the RPV model though the measurements in the principal plane (a) and all measurements covering the full hemisphere (b).

A regression analysis indicated that there was a strong relationship between the RPV parameters and the SMC level. In figure 2.8a this relationship is shown at 1998 $\mathrm{nm}$. The $\mathrm{R}^{2}$ value between the parameters and the SMC level decreased when all samples were considered together (figure 2.8b). However, the $\mathrm{R}^{2}$ for the relationship between the $\Theta$-parameter and the SMC level was still high with a value of 0.759 . The $\mathrm{R}^{2}$ increased to 0.817 when the RWC was considered instead of the SMC level (figure 2.8c). 

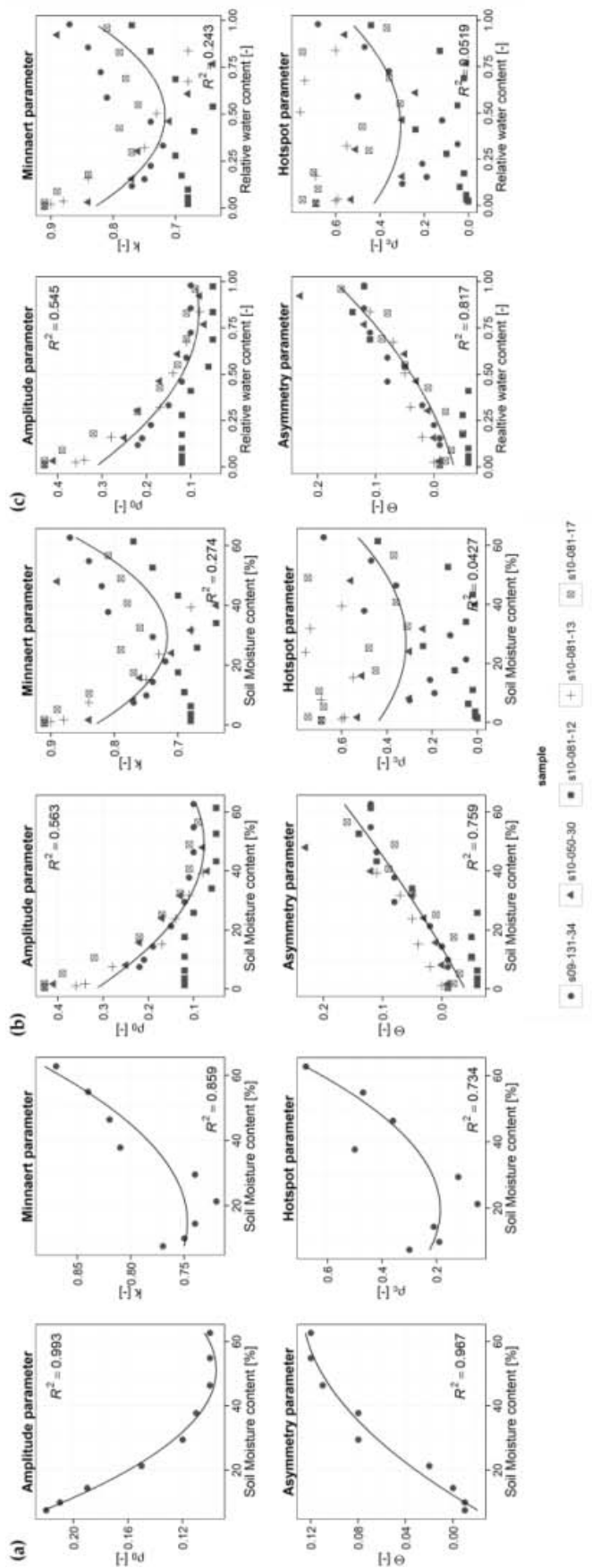

Figure 2.8. Relationship between the RPV parameters and the SMC level at $1998 \mathrm{~nm}$. (a) Sample s09-131-34 and (b) all samples used in this study (c) the relationship between the RWC and the RPV parameters for all samples. 
An overview of the $\mathrm{R}^{2}$ values between the RPV parameters and the corresponding RWC and SMC level per wavelength in the $400-2200 \mathrm{~nm}$ region is given in figure 2.9. The amplitude parameter $\left(\rho_{0}\right)$, the parameter that is closely related to the surface reflectance intensity, had an $\mathrm{R}^{2}$ around 0.1 in the VNIR region of the spectrum. The $\mathrm{R}^{2}$ increased in the SWIR region with peaks in correlation between the $\rho_{0}$ parameter and SMC level around the water absorption bands at $1450 \mathrm{~nm}$ and $1900 \mathrm{~nm}$, indicating that most information based on reflectance intensity is located in these regions. The Minnaert parameter $(k)$, which controls the bowl- or bellshape of the anisotropy curve, showed a noisy pattern in $\mathrm{R}^{2}$ in the $400-1800 \mathrm{~nm}$ region. Around the water absorption band at $1900 \mathrm{~nm}$ there was an increase in $\mathrm{R}^{2}$. The asymmetry parameter $(\Theta)$, which controls the proportion of forward and backward scattering, had an overall high $\mathrm{R}^{2}$ with RWC and SMC level in the NIR and SWIR, with a slightly higher $\mathrm{R}^{2}$ around the water absorption bands. Here, we observed a maximum $\mathrm{R}^{2}$ of 0.840 and 0.823 at $1987 \mathrm{~nm}$ for the RWC and SMC level, respectively. The hotspot parameter $\left(\rho_{c}\right)$, which controls the intensity of the hotspot, had an overall low correlation with the RWC and SMC level. In the 400$2200 \mathrm{~nm}$ region the hotspot parameter showed a noisy pattern with $\mathrm{R}^{2}$ values ranging from 0.43 to close to 0 .

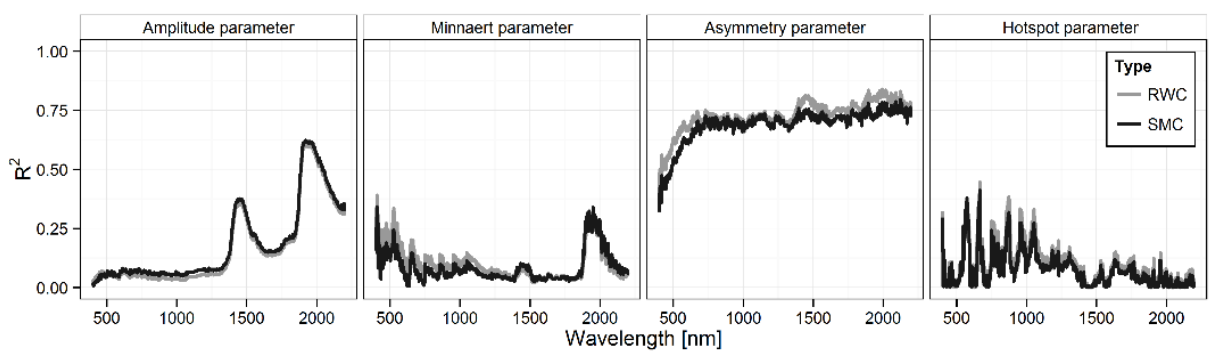

Figure 2.9. $\mathrm{R}^{2}$ between the RPV parameters and RWC or SMC level in the 400-2200 $\mathrm{nm}$ region for all soil samples combined.

\subsection{Discussion}

We studied the effects of SMC on reflectance anisotropy based on laboratory goniometer measurements and RPV model inversions. The results of the measurements clearly indicated that changes in SMC affected the anisotropic reflectance of soils. An increased SMC level caused (i) an overall decrease in reflectance (up to the point where the soils saturated), (ii) a decrease in backward scattering intensity and the hotspot effect, and (iii) an increase in forward scattering intensity.

The decrease in reflectance upon wetting that we observed in this study is in line with observations of previous studies [42,53]. Like these studies, we also observed that the reflectance in the VNIR was sensitive only to lower SMC levels, while the reflectance in the SWIR was sensitive to the whole measured range of SMC levels (figure 2.4b). The parameters obtained by the inversion of the RPV model were in 
good agreement with these observations. The amplitude parameter $\left(\rho_{0}\right)$,which has no physical meaning but is related to the intensity of the reflectance [66], had a poor correlation $\left(\mathrm{R}^{2}<0.1\right)$ with SMC in the VNIR region of the spectrum, while stronger correlations $\left(\mathrm{R}^{2}\right.$ up to 0.6$)$ were observed at wavelengths in the SWIR region (figures 2.8 and 2.9). This finding is in line with Lobell and Asner [42], who concluded that the SWIR region is more suitable for the measurement of changes in SMC due to the response of reflectance up to high SMC levels in this region.

We observed a decreased backward scattering intensity and hotspot effect and an increased forward scattering intensity in the principal plane when the SMC level increased. The increased proportion of forward scattering is in good agreement with observations of Rosendahl et al. [61]. Rosendahl et al. [61] studied the reflectance anisotropy of sand and glass granules in a laboratory experiment where they used a Sony XCL-5005 camera to capture the reflectance at different moisture levels at several positions in the principal plane and observed relatively stronger forward scattering at higher moisture levels. An explanation for this phenomenon is the decrease in relative refraction index when light travels from air to soil with the absence of water in the soil, and from air to water for conditions where water is present in the soil. The penetration of light into a medium increases when the relative refraction index between two media decreases $[47,46]$. This results in a decreased proportion of light that is directly reflected by the soil when water is present and in turn makes it more likely that the light that penetrated the soil reflects in the forward direction out of the soil [61]. This explains the higher observed proportion of forward scattering, the decreased proportion of backward scattering and the decreased hotspot effect at higher SMC levels. The RPV model could simulate the changes of the anisotropy curves in the principal plane for SMC levels ranging from air-dry to saturation point (figure 2.7). The asymmetry parameter $(\Theta)$, which controls the forward and backward scattering intensity, showed a strong correlation with the SMC level (figures 2.8 and 2.9), especially in the NIR and SWIR regions. This indicated that the forward/backwards scattering proportion in these regions contained information on the SMC level.

At oversaturated SMC levels, where Fresnel reflectance of water becomes a significant part of the reflectance signal [55,44], we observed a sun glint pattern with a maximum reflectance around the angle of specular reflectance (figure 2.6). Sun glint, the mirror-like reflectance of light at the air-water interface, occurs when the orientation of a water surface is such that light is directly reflected towards the sensor [68]. The intensity and pattern of sun glint depends on the roughness of the water surface and the illumination and viewing geometry $[69,68]$. Although no free water could be seen at the soil surface when the reflectance measurements were taken at oversaturated SMC levels, the soil particles may have been covered by a water layer that made the soil reflect like a water surface, which in turn caused the observed sun glint pattern. The RPV model does not take sun glint or specular reflectance into account and it was therefore not possible to fit the RPV model through the BCRF measurements in the principal plane at oversaturated SMC levels. In addition, at oversaturated SMC levels the observed reflectance signal is complicated, since it corresponds partly to light that is directly reflected by the water surface (Fresnel reflectance) and partly to light that penetrated and 
interacted with the soil (volumetric reflectance) [44]. Obtained RPV parameters based on the reflectance of a soil after the saturation point would therefore not correspond to SMC, but to the relative contribution of Fresnel and volumetric reflectance to the observed reflectance signal.

In literature, it was suggested that the model of Jacquemoud et al. [64] is capable of simulating the specular reflectance of soils. However, fitting this model to our data did not yield satisfactory results at oversaturated SMC levels (not shown in this paper), similar to the RPV model. Moreover, it has more empirical parameters to describe the hotspot and the forward-backward scattering ratio than the RPV model. Therefore, we focused on using the RPV model in this study.

Besides the correlation between reflectance anisotropy and the absolute volumetric soil moisture content (SMC), we also investigated the correlation between reflectance anisotropy and the relative soil moisture content (RWC). RWC is a measure that describes the relative saturation of a soil. This can have a more robust correlation with reflectance, since different soil (types) have different hydrological properties (like wilting point, field capacity and saturation point), which means that the absolute amount of water that can be held by a soil varies per soil type. The normalization from SMC to RWC might therefore be necessary to create a universal relation between soil water and reflectance anisotropy. However, the correlation of SMC and RWC with the RPV parameters showed a similar pattern with similar $\mathrm{R}^{2}$ values (figure 2.9 ).

For this study we corrected for the constantly decreasing SMC level during the timespan of the dry-out experiment by linearly interpolating the measured BCRFs for each measurement position between two consecutive SMC levels. This interpolation also enabled us to assign one SMC level to all measurements within a measurement cycle. The difference between the original reflectance factors and the interpolated reflectance factors appeared small (figures 2.5a and 2.5b). However, the best fit of parameters based on inversion of the RPV model was significantly affected by this difference (results not shown). This indicates that correcting for differences in SMC level is an important processing step for data collected in the way presented in this paper.

The spectral measurements of this study were performed under controlled laboratory conditions with constant illumination and highly accurate positioning of the sensor. Different results might be obtained if a similar experiment would be done under field conditions due to the presence of diffuse light and the changing position of the sun over time [70]. The natural presence of surface roughness and soil aggregates, which have a strong influence on reflectance anisotropy [34], were destroyed during the preparation of the samples. It might be that the anisotropic reflectance effects caused by soil surface structure and roughness overrule the effects caused by soil moisture. In this study, we looked at the effect of SMC on surface anisotropy. Confounding effects of surface roughness as well as organic matter content requires further research. In addition, the presence of vegetation, rocks etcetera was not considered in this study. This, in combination with the acquisition time of the large number of observation positions that were used in this study, limits the applicability for in situ measurements of SMC based on reflectance 
anisotropy. Further research should indicate whether a few observation angles suffice to quantify the reflectance anisotropy of soils.

The results of this study indicate a strong influence of SMC on reflectance anisotropy. Therefore, it is recommended that reflectance anisotropy is taken into account for optical remote sensing methods when analyzing data acquired under different viewing and illumination geometries. For future research we recommend to study the effects of soil moisture on the anisotropic reflectance behavior of soils while taking the natural soil texture into account. In addition, the influence of natural illumination conditions need to be studied to explore the opportunities for upscaling the presented research to an airborne or space borne level and applying it at larger spatial extents. Currently, first attempts have been made to perform multi-angular measurements from an unmanned aerial vehicle (UAV). These platforms are very agile and first results look promising for observing objects at multiple angles. In this way anisotropic reflectance information might be captured from an airborne platform.

\subsection{Conclusions}

The multi-angular reflectance measurements that were performed for this study showed that soil moisture had a strong influence on soil reflectance anisotropy. Increasing SMC levels caused a decreased proportion of backward scattering and hotspot effect, and an increased proportion of forward scattering. At oversaturated SMC levels, a sun glint reflectance pattern was observed. The RPV model was inverted against BCRF measurements that were taken in the principal plane, to obtain the RPV model parameters that best described the measured anisotropy curves at different SMC levels. Especially the asymmetry parameter $(\Theta)$, which controls the proportion of forward and backward scattering, had a strong correlation with the SMC level (maximum $\mathrm{R}^{2}$ of 0.797 at $2123 \mathrm{~nm}$ with SMC and maximum $\mathrm{R}^{2}$ of 0.840 at $1993 \mathrm{~nm}$ with RWC). The correlation between the amplitude parameter $\left(\rho_{0}\right)$, which is closely related to the reflectance intensity, and the SMC and RWC was less (maximum R ${ }^{2}$ of 0.622 at $1921 \mathrm{~nm}$ for SMC and maximum $\mathrm{R}^{2}$ of 0.604 at also at $1921 \mathrm{~nm}$ for RWC). This indicated that reflectance anisotropy contained more information on SMC than reflectance intensity.

Acknowledgements. This research was funded by a grant from the User Support Programme Space Research (GO/12-15) in The Netherlands. 



\section{Chapter 3}

\section{Hyperspectral reflectance anisotropy measurements using a pushbroom spectrometer on an unmanned aerial vehicle-Results for barley, winter wheat, and potato}

This chapter is based on:

Roosjen, P.P.J.; Suomalainen, J.M.; Bartholomeus, H.M.; Clevers, J.G.P.W. (2016). Hyperspectral reflectance anisotropy measurements using a pushbroom spectrometer on an unmanned aerial vehicle-Results for barley, winter wheat, and potato. Remote Sensing, 8(11), 909. 


\begin{abstract}
Reflectance anisotropy is a signal that contains information on the optical and structural properties of a surface and can be studied by performing multi-angular reflectance measurements that are often done using cumbersome goniometric measurements. In this paper we describe an innovative and fast method where we use a hyperspectral pushbroom spectrometer mounted on a multirotor unmanned aerial vehicle (UAV) to perform such multi angular measurements. By hovering the UAV above a surface while rotating it around its vertical axis, we were able to sample the reflectance anisotropy within the field of view of the spectrometer, covering all view azimuth directions up to a $30^{\circ}$ view zenith angle. We used this method to study the reflectance anisotropy of barley, potato, and winter wheat at different growth stages. The reflectance anisotropy patterns of the crops were interpreted by analysis of the parameters obtained by fitting of the Rahman-PintyVerstraete (RPV) model at a 5-nm interval in the 450-915 $\mathrm{nm}$ range. To demonstrate the results of our method, we firstly present measurements of barley and winter wheat at two different growth stages. On the first measuring day, barley and winter wheat had structurally comparable canopies and displayed similar anisotropic reflectance patterns. On the second measuring day the anisotropy of crops differed significantly due to the crop-specific development of grain heads in the top layer of their canopies. Secondly, we show how the anisotropy is reduced for a potato canopy when it grows from an open row structure to a closed canopy. In this case, especially the backward scattering intensity was strongly diminished due to the decrease in shadowing effects that were caused by the potato rows that were still present on the first measuring day. The results of this study indicate that the presented method is capable of retrieving anisotropic reflectance characteristics of vegetation canopies and that it is a feasible alternative for field goniometer measurements.
\end{abstract}




\subsection{Introduction}

Natural surfaces reflect light anisotropically, which means that the reflected radiance varies with viewing and illumination geometry. Reflectance anisotropy is described by the bidirectional reflectance distribution function (BRDF), which is a function that quantifies the surface reflectance into a specific direction when being illuminated from a particular direction [4]. For remote sensing purposes, information on the BRDF is important, for example, for the correction of viewing and illumination effects [71] or for the calculation of albedo [72,11]. In addition to this, the BRDF has been shown to contain additional information on vegetation parameters, such as canopy density, foliar water content, nitrogen content and leaf area index [73], canopy height [74], canopy clumping [75,76] and soil properties, such as soil surface roughness [34] and soil moisture content [77]. Moreover, BRDF information can be used to improve classification accuracies [78].

Reflectance anisotropy is commonly studied by performing multi-angular reflectance measurements using goniometers in laboratories under controlled conditions e.g., $[20,21,15,7]$ or in the field under natural conditions e.g., $[79,24,25,39,27]$. Both laboratory and field goniometer measurements have their advantages and disadvantages [80]. A drawback of using laboratory goniometry is that the observed target has to be taken out of its natural environment and that an artificial light source has to be used, which typically results in a non-parallel light beam as opposed to the natural illumination of the sun [81]. A positive aspect, on the other hand, is the full control over the position and stability of the light source during measurement acquisition. An advantage of field goniometry is that the target can be observed in its natural environment and under natural illumination conditions. However, this also has as a consequence that measurements are affected by influences of atmospheric conditions, wind, and changes in illumination conditions due to the movement of the sun and clouds [82]. A drawback of both laboratory and field goniometer measurements is that they are cumbersome and time consuming.

Unmanned aerial vehicles (UAVs) might provide more elegant opportunities for performing multi-angular reflectance measurements. The fast emergence and development of unmanned aerial platforms and lightweight optical sensors has recently boosted the availability of new techniques for acquiring spectral data [83]. The relatively low operation height of UAVs results in derived products with a small spatial pixel size [84], which are typically interesting for small scale precision agriculture applications $[85,86,19]$. UAVs have already become common platforms for use in remote sensing [35], but the number of studies that use UAVs to perform multi angular measurements for investigation of reflectance anisotropy is still small.

Until now, only a few approaches of using a UAV to capture reflectance anisotropy have been explored. Hakala et al. [38] used a consumer camera mounted on a UAV to acquire reflectance anisotropy of snow-covered land surfaces and compared these with simultaneous ground measurements. Grenzdörffer and Niemeyer [37] also used a consumer-grade camera mounted on a UAV to study the anisotropic 
reflectance behavior of winter wheat fields by capturing images at different view zenith and azimuth angles covering the full hemisphere. Burkart et al. [36] used a hyperspectral UAV-based pointable spectrometer to collect multi-angular data of several vegetated areas and compared the measurements with the SCOPE model. Honkavaara et al. [87] have demonstrated a method for extracting the reflectance anisotropy from multi/hyperspectral frame images in the georectification process. Duan et al. [88] collected hyperspectral images of several crop types during two consecutive flights performed in different directions using a UAV, which resulted in pixels that were observed from two directions in the overlapping part of the two flight lines. Based on this dual angle dataset Duan et al. showed an improvement in the estimation of the leaf area index (LAI). Reflectance anisotropy is also reported as an unwanted effect: Rasmussen et al. [89] investigated the performance of different vegetation indices using a consumer-grade camera mounted on a UAV and observed strong angular variation in reflectance images acquired in sunny conditions. To avoid these anisotropic effects, they advised to acquire data during clouded days. Not only viewing geometry, but also illumination geometry, can have an influence on anisotropic reflectance effects. Brede et al. [90] studied the effect of solar zenith angle on the enhanced vegetation index (EVI) and found a linear trend in the decrease of the EVI with an increasing illumination zenith angle.

In this paper we present an innovative method to rapidly measure reflectance anisotropy using a UAV and a hyperspectral pushbroom spectrometer system. We demonstrate the method by two showcases: (1) we compare anisotropy measurements of barley and winter wheat, two different crops that are structurally comparable due to their vertically-oriented canopies. In addition, we show how differences in grain head development of both crops result in different anisotropy patterns; and (2) we show the effect of canopy development on reflectance anisotropy for potato crops by measurements before and after the potato canopy closed.

\subsection{Materials and methods}

\subsubsection{Study area and flight pattern}

The study area consisted of three fields with different crops located north of Wageningen, the Netherlands $\left(51^{\circ} 59^{\prime} 34.8^{\prime \prime} \mathrm{N}, 5^{\circ} 39^{\prime} 4.6^{\prime \prime} \mathrm{E}\right)$. The study area was characterized as an open and flat agricultural area without any vertical elements nearby. The target crops were barley (Hordeum vulgare L., sowing date: 18 March 2015), potato (Solanum tuberosum L., sowing date: 14 April 2015), and winter wheat (Triticum aestivum L., sowing date: 21 October 2014), which were growing in three adjacent fields (figure 3.1).

Several UAV flights were performed over the study area at two different growth stages of the crops during the summer of 2015 (table 3.1). All measurements were taken under clear sky conditions to minimize the variance in diffuse illumination and to ensure the strongest directional effects. 

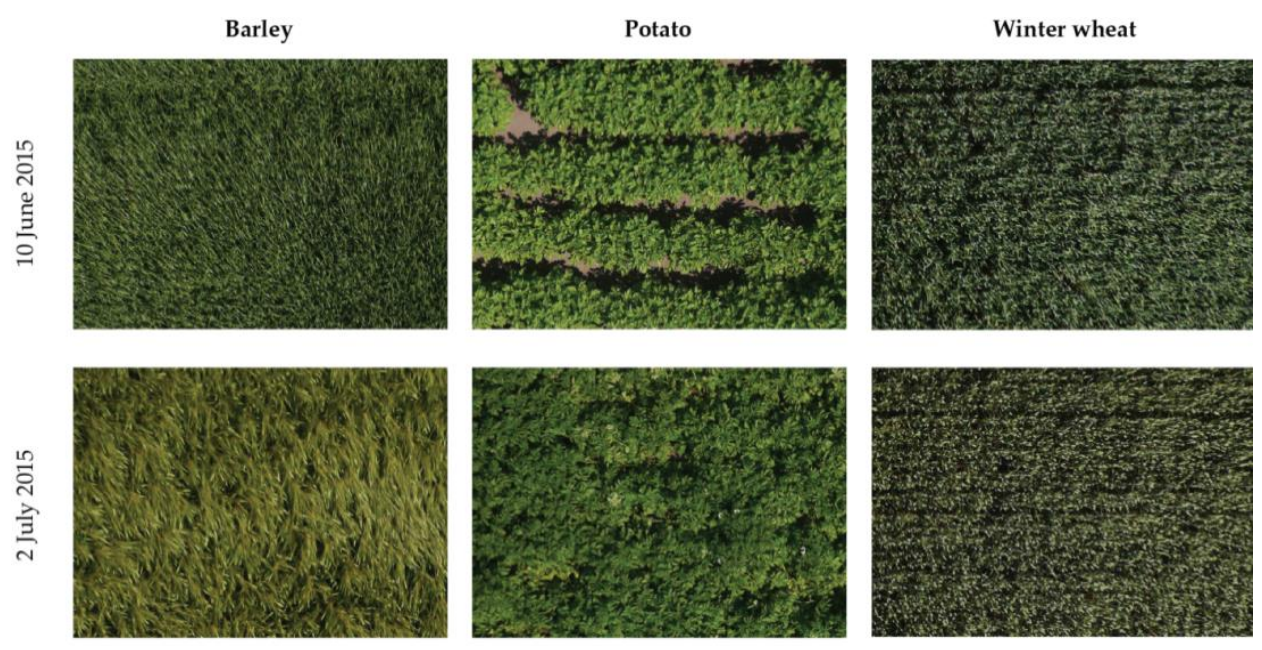

Figure 3.1. Photographs taken from the UAV of the barley, potato, and winter wheat targets during the two measurement days.

The UAV measurements were performed using the Wageningen UR Hyperspectral Mapping System (HYMSY) on board an Altura AT8 octocopter [91], figure 3.2. The HYMSY at its current configuration consists of a pushbroom spectrometer (450$915 \mathrm{~nm}, 9 \mathrm{~nm}$ full-width-at-half maximum (FWHM), 25 lines/s, 328 pixels/line), a photogrammetric camera, and a miniature GPS-inertial navigation system (INS). The system and processing chain are specifically designed for agricultural mapping and monitoring applications of small areas (2-10 ha) in fine spatial detail.

Table 3.1. Solar position at the time of data acquisition. The times are local time (UTC $+2 \mathrm{~h}$ )

\begin{tabular}{ccccccccc}
\hline \multicolumn{3}{c}{ Day 1: 10 June 2015 } & & \multicolumn{3}{c}{ Day 2: 2 July 2015 } \\
\cline { 1 - 3 } \cline { 6 - 8 } Flight \# & Time & Azimuth & Zenith & & Flight \# & Time & Azimuth & Zenith \\
\hline 1 & $10: 00$ & $104^{\circ}$ & $50^{\circ}$ & & 3 & $10: 00$ & $103^{\circ}$ & $51^{\circ}$ \\
2 & $10: 30$ & $111^{\circ}$ & $46^{\circ}$ & & 4 & $10: 30$ & $110^{\circ}$ & $47^{\circ}$ \\
\hline
\end{tabular}

The HYMSY is typically flown with the scanline of the spectrometer perpendicular to the flight direction and it produces a digital surface model (DSM) and a hyperspectral geocoded datacube, using orientations fused from the INS data and the photogrammetric orientation of the camera images. For the measurements of this study we took a different approach. Instead of performing the flight while maintaining the scanline of the spectrometer perpendicular to the flight direction, we flew the UAV while rotating it around its vertical axis at a constant rotation speed of approximately one revolution in $10 \mathrm{~s}$. In this way, the azimuth orientation of each collected scanline was in a different azimuth plane. Based on the INS data and the photogrammetric orientation of the aerial images we derived the observation azimuth and zenith angles for each pixel. Collecting scanlines while completing full rotations with the UAV resulted in datasets covering the full $360^{\circ}$ 
azimuth sampling range. While most goniometers sample the same area from multiple observation directions, the method presented in this paper takes multiple pixels in a sampling area from multiple observation directions. The rationale behind this is that, if the sampling area contains only a single crop or vegetation type and a large amount of pixels are measured, the measurements of the individual pixels will represent the reflectance anisotropy of the sampling area. The measurement principle can be compared to tower-based multi-angular measurements where different azimuth and zenith angles are sampled by rotating a sensor at a fixed position above a surface, such as the case of the Automated Multiangular Spectro-radiometer for Estimation of Canopy reflectance (AMSPEC) series [28-30] or the Portable Apparatus for Rapid Acquisition of Bidirectional Observation of the Land and Atmosphere III (PARABOLA III) [31].

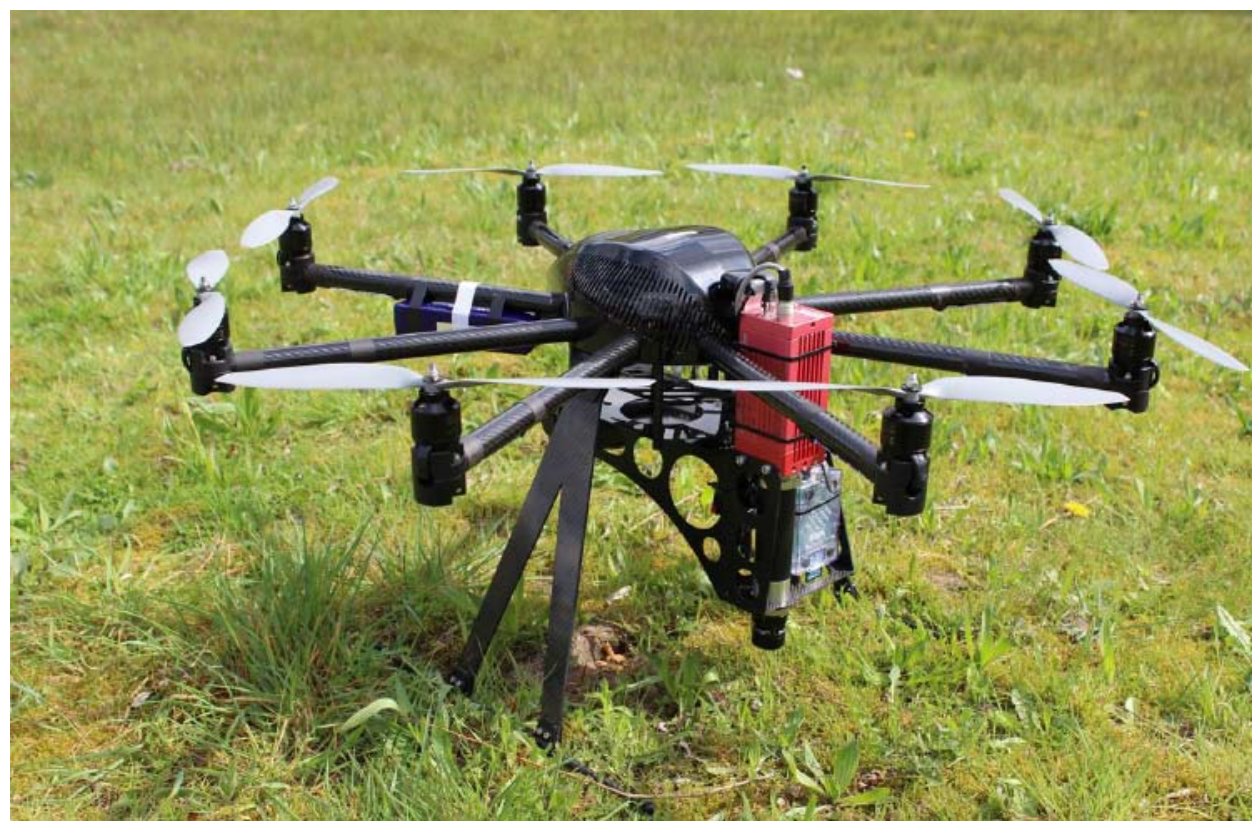

Figure 3.2. The Aerialtronics Altura AT8 UAV and the sensor system.

The field of view (FOV) of the spectrometer's scanline that was used for the measurements Was $42.4^{\circ}$. When rotating the UAV while the center of the FOV is aimed towards the nadir position, the maximum zenith sampling angle would be $21.2^{\circ}$ To increase the maximum zenith sampling angle, we mounted the spectrometer tilted under the UAV to capture pixels at greater zenith angles (figure 3.3a). The actual maximum zenith angle sampling range depends on the roll, pitch and yaw of the UAV during the flights and is, therefore, slightly different for each scanline.

For the data collection, the UAV was programmed to hover for one minute at a height of approximately 10 meters above ground level at waypoint that was selected in the center of each of the fields. Operating at this height resulted in a 
ground sampling distance of about $6 \mathrm{~cm}$. Once the UAV completed a $360^{\circ}$ rotation around its vertical axis, a theoretically circular sampling area of approximately 100 $\mathrm{m}^{2}$ was covered. However, the rotational instability of the UAV resulted in a rotation around the waypoint, forming a ring-shaped sampling area (figure 3.3b). Transforming the measurement scheme of figure 3.3a to a polar coordinate system results in an angular sampling range up to around $30^{\circ}$ zenith covering all azimuth directions (figure 3.3c). The scanlines of figure 3.3b result in a coverage of azimuth and zenith angles as shown in figure $3.3 \mathrm{~d}$.

(a)

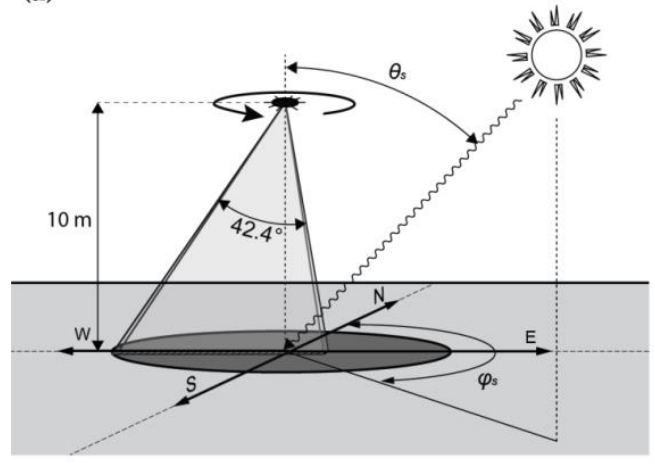

(c)

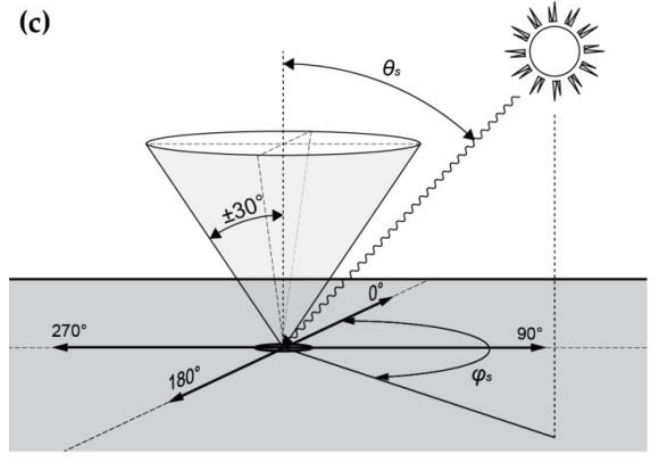

(b)

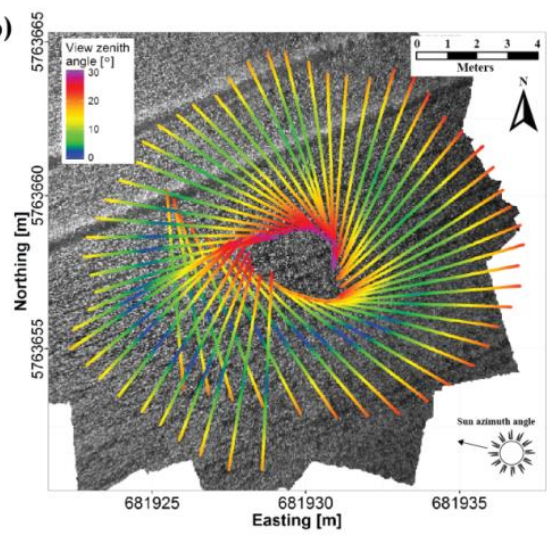

(d)

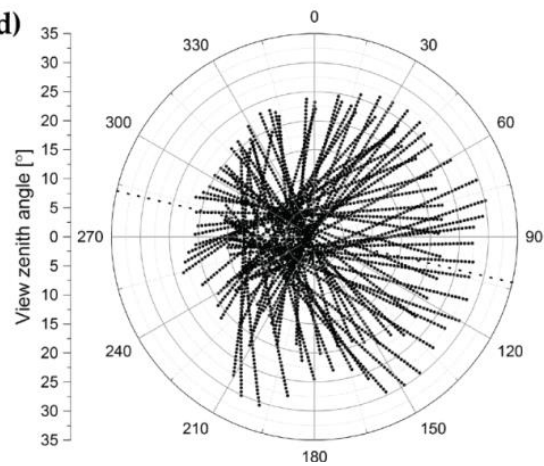

Figure 3.3. Illustration of the measurement principle shown by the zenith angle sampling range when rotating the UAV with the tilted spectrometer (a) and the actual view zenith angles of the scanlines collected during one full rotation over the winter wheat canopy on 2 July 2015 10:00 a.m. in a WGS84 UTM 31N projection (b). For clarity, only every tenth collected scanline is displayed. A goniometric representation of the sampling range (c) and the azimuth and zenith angles of the scanlines of figure $3.3 \mathrm{~b}(\mathrm{~d})$ are also depicted. The dotted line in figure $3.3 \mathrm{~d}$ indicates the solar principal plane

The final collected datasets per crop consisted of 230,000-350,000 pixels within each sampling area at 94 spectral bands, ranging from 450 to $915 \mathrm{~nm}$. In the fields there were some tractor tracks (figure 3.3b), which for some targets and view directions formed a significant part of the sampled area. These tracks were 
manually filtered out before processing of the data. For the barley and winter wheat targets the observed anisotropy patterns could, therefore, be assigned solely to the crops. For the potato crops on the first measuring day, the anisotropy pattern is a combination of the signal of the potato plant and the soil background due to the open row structure of the potato canopy at this time (figure 3.1).

\subsubsection{Data processing}

The radiometric and geometric processing of the UAV data was done using the standard HYMSY processing chain described in detail in [91]. In this processing chain, the radiances measured by the spectrometer were converted to reflectance factors using the empirical line method with a measurement of a $25 \mathrm{~cm} \times 25 \mathrm{~cm}$ $25 \%$ grey Spectralon panel (LabSphere Inc., North Sutton, NH, USA) and a dark current measurement taken just prior to the flight. To improve the signal-to noise ratio, we applied Gaussian smoothing with a FWHM of 5 bands (25 nm) to the spectra.

For the geometric alignment of the HYMSY, aerial photos collected during the flights were aligned in Agisoft PhotoScan software (v1.1.2, AgiSoft LLC, St. Petersburg, Russia). The photogrammetric alignment of the photos was then fused with the data from HYMSY GPS INS to calculate view zenith and azimuth angles of each pixel. In the standard HYMSY processing these geometric metadata would have been used for the georectification of the hyperspectral data but, for the reflectance anisotropy measurements presented here, this step was omitted.

Due to the low accuracy of the magnetic compass in the HYMSY system and the lack of horizontal movement during the flights to exploit GPS data for sensor headings, the accuracy of azimuth angles retrieved from the standard HYMSY processing was found to be inadequate. Therefore, it was still mandatory to manually define the solar principal plane in the reflectance anisotropy data and use that to define the view azimuth angles. The accuracy of the final azimuth and zenith angles was estimated to be better than $2^{\circ}$. Usage of ground control points in the experiment would have resulted in better angular accuracy and removed the need for manual correction.

\subsubsection{Data analysis}

The reflectance anisotropy was quantified using the anisotropy factor (ANIF). The ANIF is a ratio that describes the reflectance factor relative to the nadir reflectance factor [7]:

$$
\operatorname{ANIF}\left(\lambda, \theta_{i}, \varphi_{i}, \theta_{r}, \varphi_{r}\right)=\frac{R\left(\lambda, \theta_{i}, \varphi_{i}, \theta_{r}, \varphi_{r}\right)}{R_{0}\left(\lambda, \theta_{i}, \varphi_{i}, 0^{\circ}, 0^{\circ}\right)}
$$

where $R$ is the reflectance factor at wavelength $\lambda$ observed from direction $\left[\theta_{r}, \varphi_{r}\right]$ and illuminated from direction $\left[\theta_{i}, \varphi_{i}\right] . R_{0}$ is the reflectance factor at the nadir view. 
To smoothen and filter the measurements, the data were resampled to a discrete grid of $1^{\circ}$ azimuth and zenith view angles. We applied an angular smoothing of an area of $5^{\circ}$ radius around each discrete azimuth and zenith observation angle to be able to assign a single reflectance factor to an observation position. A discrete azimuth and zenith observation angle was only considered if there were at least 1,000 pixels within this $5^{\circ}$ radius.

To interpret the measurements and parameterize the anisotropy patterns, we fitted the Rahman-Pinty-Verstraete model (RPV) [66] through each dataset. The RPV model is a semi empirical model that uses four parameters to simulate the BRDF of any arbitrary surface. It has been used in anisotropy studies of a wide variety of targets like forests [92], grasslands and cultivated fields [93], snow [38], and soil $[77,94]$ at different scales ranging from the centimeter scale in laboratory studies $[95,77]$, to the decimeter scale in UAV- and airborne-based studies $[38,78]$, up to a scale of several hundreds of meters to a kilometer scale in space-borne studies. The model splits the reflectance anisotropy in an amplitude component $\left(\rho_{0}\right)$ and a directional component:

$$
\rho_{s}\left(\theta_{i}, \varphi_{i} ; \theta_{r}, \varphi_{r}\right)=\rho_{0} \frac{\cos ^{k-1} \theta_{i} \cos ^{k-1} \theta_{r}}{\left(\cos \theta_{i}+\cos \theta_{r}\right)^{1-k}} F(g)[1+R(G)]
$$

where the $k$ parameter controls the bowl/bell shape of the anisotropy curve. The relative amount of forward/backward scattering, controlled by the $\Theta$ parameter, is defined as:

$$
F(g)=\frac{1-\Theta^{2}}{\left[1+\Theta^{2}-2 \Theta \cos (\pi-g)\right]^{1.5}}
$$

where

$$
g=\cos \theta_{i} \cos \theta_{r}+\sin \theta_{i} \sin \theta_{r} \cos \left(\varphi_{i}-\varphi_{r}\right)
$$

The hotspot is approximated by:

$$
1+R(G)=1+\frac{1-\rho_{c}}{1+G}
$$

where

$$
G=\left[\tan ^{2} \theta_{i}+\tan ^{2} \theta_{r}-2 \tan \theta_{i} \tan \theta_{r} \cos \left(\varphi_{i}-\varphi_{r}\right)\right]^{1 / 2}
$$

To estimate the parameters that best describe the anisotropy, the RPV model was fitted through the multi-angle reflectance measurements by a non-linear least squared regression method that uses the Levenberg-Marquardt algorithm, implemented in $R$ (version 3.3.1) [96]. The range of possible solutions for the fitting of the RPV model for the $\Theta$ parameter was restricted between -1 and 1 , where $\Theta<0$ 
indicates predominant backward scattering and $\Theta>0$ indicates predominant forward scattering anisotropy. Since no measurements were taken close to the hotspot (table 3.1), the $\rho_{c}$ parameter was fixed at 1 , which indicates no additional fitting around the hotspot position. The range of the $k$ parameter was left free, where $k<1$ resembles a bowl shaped anisotropy curve and $k>1$ resembles a bellshaped anisotropy curve.

\subsection{Results and discussion}

\subsubsection{Barley and winter wheat}

On the first measuring day, when the top layer of the barley and winter wheat canopies were still formed by leaves, both the barley and winter wheat displayed typical anisotropy characteristics, as are commonly seen for vertically-oriented canopies. In the red band $(650 \mathrm{~nm})$, where shadows are darkest due to absorption of radiance by chlorophyll, the lowest ANIFs were observed in the forward scattering direction and the highest ANIFs in the backward scattering direction (figure $3.4 \mathrm{~b}, \mathrm{e}$ ).

\section{Barley 10 June 2015 10:00 a.m.}

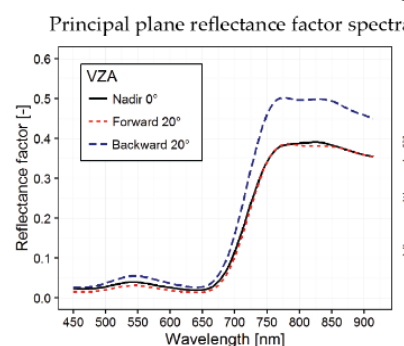

(a)

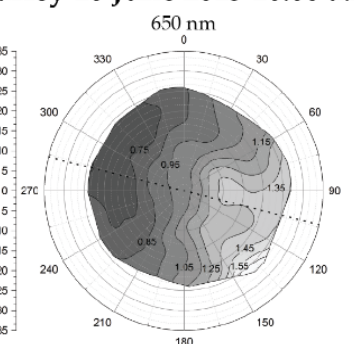

(b)

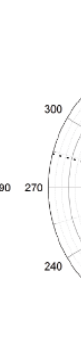

(1)

Winter wheat 10 June 2015 10:00 a.m.

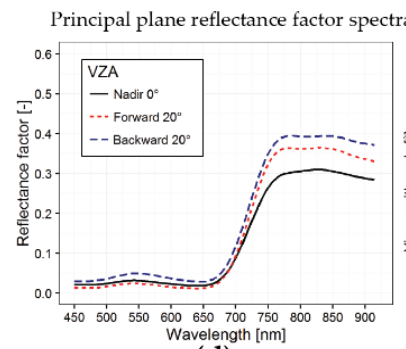

(d)

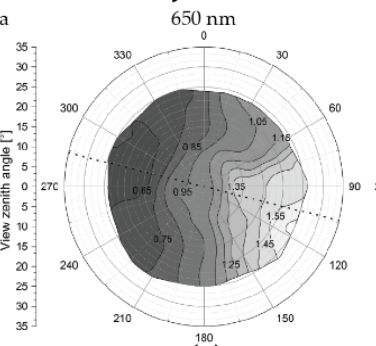

(e)

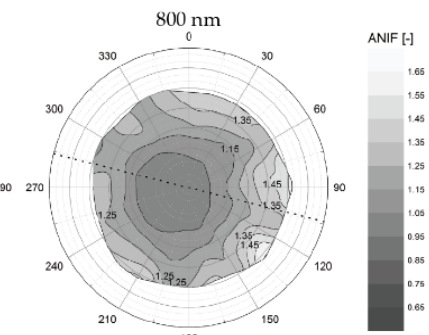

(f)

Figure 3.4. Barley (top) and winter wheat (bottom) measurements collected during flight 1 (10 June 2015 at 10:00 a.m., table 3.1). Spectra in the forward and backward scattering direction and at nadir (a, d). Interpolated polar plots at $650 \mathrm{~nm} \mathrm{(b,} \mathrm{e)} \mathrm{and}$ $800 \mathrm{~nm}(\mathrm{c}, \mathrm{f})$. The dotted lines indicate the solar principal plane at the time of data acquisition. 
This effect occurs because, in the backward scattering direction, the sensor was viewing the well-illuminated side of the canopies, which resulted in a higher reflectance, and in the forward scattering direction the shadowed side of the canopies, which resulted in a lower reflectance. In addition to this effect, view zenith angles (VZAs) close to nadir allow for a view of the lower shadowed layers of the canopy and with increasing off-nadir VZAs the relative proportion of higher, well-illuminated canopy layers that are observed by the sensor increases, resulting in an increase of reflectance [6,7].

In the near-infrared band (NIR, $800 \mathrm{~nm}$ ), where shadow effects are weaker due to lower absorption of radiance by chlorophyll, the ANIFs increased with increasing view zenith angles, independent of the view azimuth direction. The small contribution of the shadow effect puts the position of the lowest observed ANIF close to nadir, slightly in the forward direction (figure 3.4c,f).

The $k$ and $\Theta$-parameter spectra, obtained at a 5-nm interval between $450 \mathrm{~nm}$ and $915 \mathrm{~nm}$ by fitting the RPV model through the measurements of flights 1 and 3 on day 1 and day 2 at 10:00 a.m. (table 3.1), illustrate the observed anisotropy patterns in terms of the reflectance bowl/bell shaped anisotropy and forward/backward scattering dominance, respectively (figure $3.5 \mathrm{a}-\mathrm{c}$ ). On the first measuring day, both crops were in a pre-heading stage and the top layers of their canopies were mainly formed by leaves. For both crops at this stage, the $k$ parameter spectrum followed a trend opposite to that of a vegetation reflectance factor spectrum (figure 3.5b). The blue and red wavelengths had $k>1$, indicating a bell-shaped anisotropy curve. At these wavelengths the anisotropy was dominated by shadow effects due to the strong absorption of radiation by chlorophyll in the leaves that formed the top layer of the barley and winter wheat canopies. Accordingly, this resulted in the lowest values of $\Theta$ in these wavelength regions (figure 3.5c). In the green part of the spectrum these shadow effects were weaker due to less absorption by chlorophyll in this region, resulting in slightly higher $\Theta$ values and $k$ values just below $1 . k$ values $<1$ were obtained by fitting the RPV model in the NIR wavelength region, indicating a bowl-shaped anisotropy pattern. Moreover, the backward scattering was weaker in this wavelength region, which resulted in $\Theta$ values of roughly -0.13 for barley and -0.09 for winter wheat between 750 and $915 \mathrm{~nm}$ (figure 3.5c). Even though barley and winter wheat are different crops, both of them displayed very similar anisotropy patterns over the measured spectral domain on the first measurement day due to their similar canopy structures.

At the time of the second measuring day, both crops were in their grain development stage. This had as a result for barley that the top layer of the canopy was no longer formed by leaves, but by a layer of less photosynthetically active grain heads with large awns and the heads were clearly bending downwards (figure 3.1). The anisotropy at this stage was characterized by an overall increased $k$ parameter value at all measured wavelengths with $k>1$ in the visible wavelength region (figure 3.5e). In addition, the backward scattering intensity decreased in the visible wavelengths with $\Theta$ values of around -0.33 to approximately -0.12 (figure $3.5 \mathrm{c}, \mathrm{f})$. 
The winter wheat canopy on the second measuring day was formed by verticallyoriented grain heads that were pointing out above the leaf layer, also resulting in less photosynthetically active material in the a top canopy layer. This resulted in a similar $k$ spectrum as for the barley canopy (figure 3.5e). The $\Theta$ parameter followed the same trend as during the first day, but with a less emphasized peak at green wavelengths. In the NIR wavelength region, the $\Theta$ parameter decreased from -0.09 to -0.16 , indicating stronger shadow effects due to the presence of opaque grain heads in the top canopy layer.

Day 1: 10 June 2015 10:00 a.m.

Day 2: 2 July 2015 10:00 a.m.

(a)

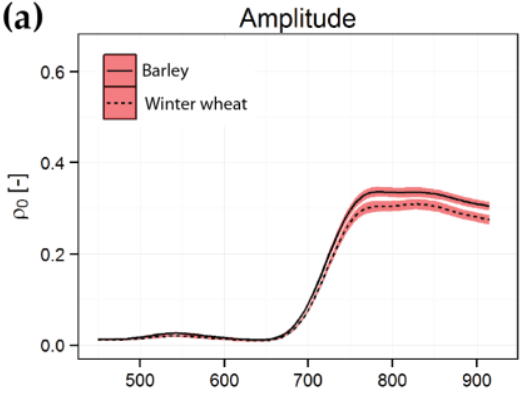

(b)

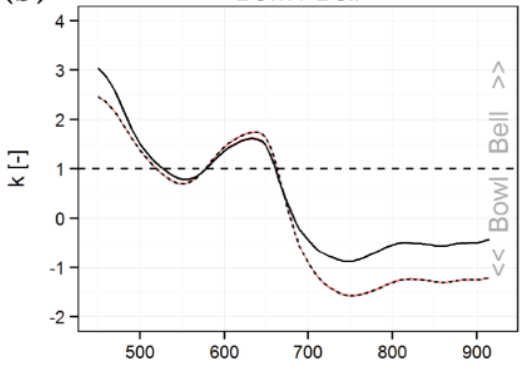

(c)

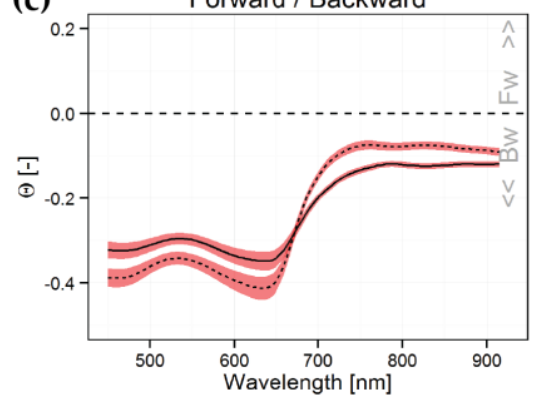

(d)

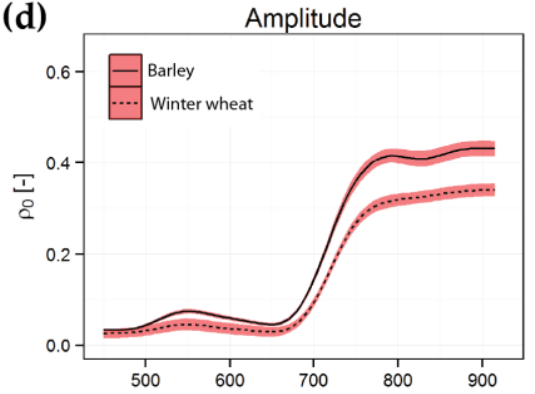

(e)

Bowl / Bell

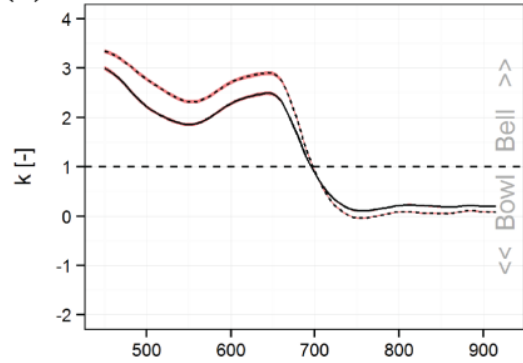

(f)

Forward / Backward

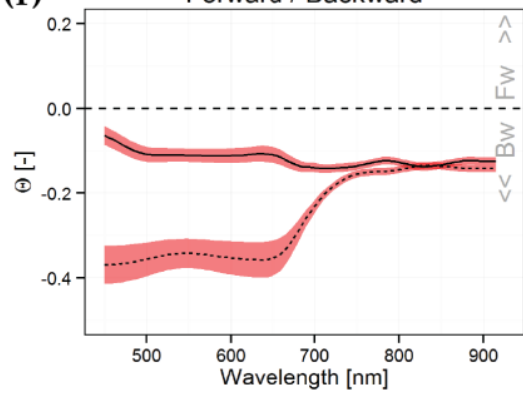

Figure 3.5. Estimates of the $\rho_{0}(\mathrm{a}, \mathrm{d}), k$ (b,e), and $\Theta$ (c,f) parameter values from 450 to $915 \mathrm{~nm}$, obtained by fitting the RPV model through the barley and winter wheat measurements of the flights at 10:00 a.m. on 10 June 2015 and 2 July 2015. The red areas indicate the estimated standard error of the estimated parameter values. 


\subsubsection{Potato Canopy Closure}

On measurement day 1 , when the potato canopy was showing an open row structure, the soil formed a significant part of the signal when measured by the sensor in the nadir view. With increasing VZA, the proportional area of observed soil decreased and the proportional area of observed vegetation increased. In the red wavelength region, an increase in VZA resulted, therefore, in a decrease of the reflectance factor, since soil has a higher reflectance at this wavelength compared to vegetation. In addition, the row structure of the potato crops produced strong shadows, which caused a higher reflectance in the backward scattering direction and lower reflectance in the forward scattering direction. This resulted in a bellshaped curve with the highest ANIFs around the nadir and in the backward scattering direction (Figure 3.6b). Bell-shaped anisotropy curves are typically seen at bright surfaces that are partially covered by darker objects, such as sparse agricultural canopies [6] or snow-covered surfaces with snow-free trees [92]. In the NIR wavelength region an increase in VZA resulted in an increased ANIF, which is opposite to the red region, since vegetation has a higher reflectance in this wavelength region than soil.

Between measurement days 1 and 2, the potato canopy grew from an open row structure to a continuous surface covering the soil almost completely (figure 3.1). In addition to a strong increase of the amplitude parameter in the NIR due to the increased vegetation cover (figure 3.7a), this resulted in a significantly decreased reflectance anisotropy over the full measured wavelength region on day 2 . Especially, we observed a strong decrease in backward scattering, which is clearly indicated by the increase of the $\Theta$ parameter to values close to 0 between day 1 and day 2 (figure 3.7c). 
Potato day 1: 10 June 2015 10:30 a.m. - Open row structure

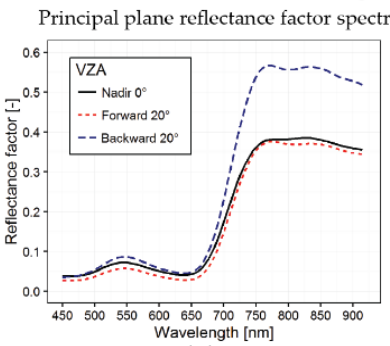

(a)

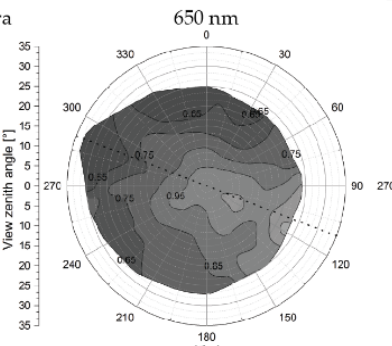

(b)

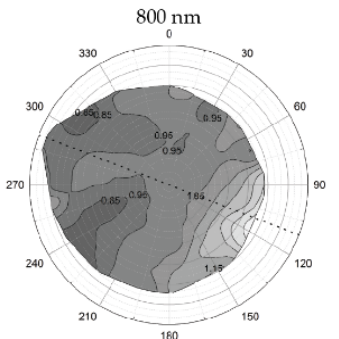

(c)

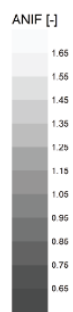

Potato day 2: 2 July 2015 10:30 a.m. - Closed canopy surface

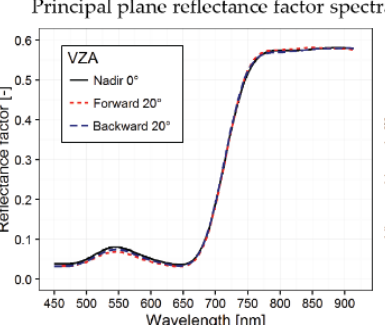

(d)

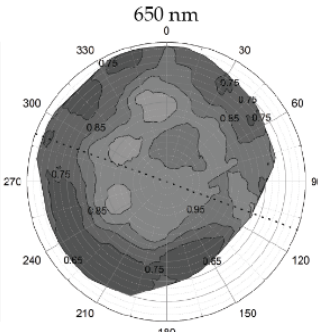

(e)

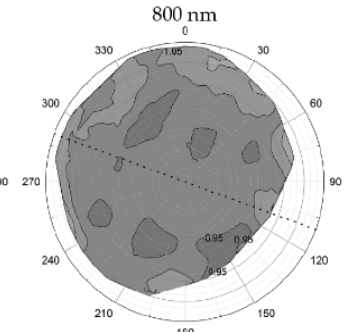

(f)

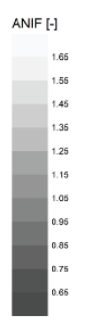

Figure 3.6. Open (top) and closed (bottom) potato canopy. Spectra in the forward and backward scattering direction and at nadir $(\mathrm{a}, \mathrm{d})$. Interpolated polar plots at 650 $\mathrm{nm}(\mathrm{b}, \mathrm{e})$ and $800 \mathrm{~nm}(\mathrm{c}, \mathrm{f})$. The dotted line indicates the solar principal plane at the time of data acquisition.

The decrease in backward scattering is likely due to the disappearance of the open row structure between day 1 and day 2 and, thus, the absence of strong rowinduced shadows. The general bowl/bell anisotropy pattern remained nearly the same between day 1 and 2, as can be seen by the similar k parameter spectra on both days (figure 3.7b). However, the bowl shape was less pronounced in the 750$915 \mathrm{~nm}$ region as can be seen by the increase of the $k$ values from around 0 to 0.58 on day 1 and 2, respectively. Likely, this happened because the less reflective soil in the NIR that was observed by the sensor on measurement day 1 at near nadir VZAs was no longer observed on day 2, resulting in a weaker increase of reflectance with increasing VZAs in this wavelength region. 


\section{Potato day 1 and 2}
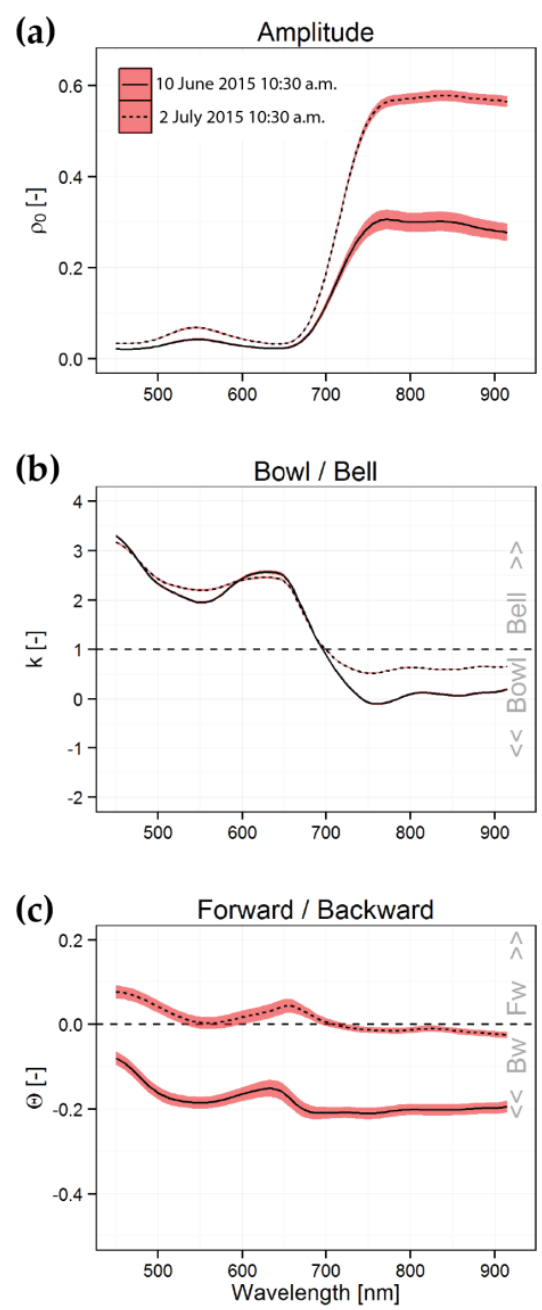

Figure 3.7. Estimates of the $\rho_{0}(\mathrm{a}, \mathrm{d}), k$ (b,e), and $\Theta$ (c,f) parameter values from 450 to $915 \mathrm{~nm}$, obtained by fitting the RPV model through the measurements of the potato canopy at day 1 and day 2 at 10:30 a.m. The red areas indicate the estimated standard error of the estimated parameter values.

\subsubsection{General discussion}

We presented an innovative and fast method in which we captured reflectance anisotropy by extracting the multi-angular views that are collected by the HYMSY system. By hovering a UAV with a hyperspectral pushbroom spectrometer above a surface while rotating it around its vertical axis, we were able to obtain the reflectance anisotropy of several crops. As demonstrated, the described method can be used to rapidly acquire the reflectance anisotropy. It might be an interesting 
alternative to field goniometer measurements and is especially interesting for measurements of small canopies like those of agricultural crops.

The barley and winter wheat canopies on both measuring days and the potato canopy on the second measuring day were nearly completely covering the complete surface and, therefore, the anisotropy patterns that we observed for these targets can be attributed solely to the crops. The potato canopy on the first measuring day was not completely covering the surface. The observed anisotropy signal on this day was, thus, composed by both the potato plants and the exposed soil. The measurements of the potato crop on the first measuring illustrated how the row structure of the potato plants resulted in strong differences between forward and backward scattering due to the presence of shadows. The closing of the potato canopy resulted in an overall decrease of reflectance anisotropy, and especially in a decrease of backward scattering intensity, something that has also been observed with canopy closure of soybeans [97]. The anisotropy patterns of barley and winter wheat were dominated by backward scattering over the full measured spectral domain, with emphasis on the visible wavelength region, combined with a strong bell-shaped anisotropy in the NIR. Similar patterns for barley and wheat were also found in several other studies e.g., [98-100] and are typical for vertically-oriented vegetation canopies, such as grasses e.g., [15,7].

With our sensor hardware and additional tilt of the spectrometer, the maximum VZA was limited to $25^{\circ}-35^{\circ}$, which matches the VZAs of most satellite and airborne remote sensing sensors. Very likely, extending the VZA to larger angles would provide more information on the reflectance anisotropy and would make the fitting of the RPV model, or any other BRDF model, more stable. Tilting the spectrometer at an even greater angle would result in sampling of larger VZAs. However, it has to be taken into account that a larger target surface is needed to make sure that the FOV of the sensor will stay within the target boundaries. The fields in our study area were quite narrow $( \pm 50 \mathrm{~m})$ and to ensure that the sensor was only observing a single crop during the rotation of the UAV, we did not tilt the spectrometer any further, but we will experiment with extending the VZA in future measurements.

The strength of the presented method is the very high density of samples that are collected in a short amount of time. Since each of the pixels in the scanline of the spectrometer can be considered as an individual sample, this means that, per second, $328 \times 25=8,200$ samples are collected at a 5-nm interval in the 450-915 $\mathrm{nm}$ range. By performing an angular smoothing as described in the methods section, the average anisotropy of a surface can be obtained, whereas most goniometers only provide the anisotropy of a single point, which does not have to be representative for the investigated target surface. Another strength of the data collected with our method is that it contains reflectance anisotropy at canopy level over the full spectrum. From the fitting of the RPV model, we can see that the changes in bowl/bell $(k)$ and forward/backward $(\Theta)$ spectra mostly follow the changes of the normal reflectance factor spectrum. However, the relation does not seem to be linear, as sometimes small changes in nadir reflectance factor, for example in the green wavelength region, seem to produce disproportionally large changes in RPV parameters. The results indicate that the reflectance anisotropy 
signal contains information about the canopy beyond the extent of the basic (nadir) reflectance factor spectrum. Using our spectra it is also possible to evaluate the number of spectral bands necessary to generally describe the reflectance anisotropy of vegetation. Our RPV parameter spectra suggest that separate measurements in at least blue/UV, green, red, and a single NIR band should be sufficient to broadly describe the reflectance anisotropy of vegetation targets in the VIS-NIR range. Such a generic description of reflectance anisotropy should be sufficient for BRDF correction of imagery and generic algorithms extracting canopy structural parameters. For more advanced analysis of reflectance anisotropy, there still may be benefits in collecting full hyperspectral data. An interesting approach to reflectance anisotropy is to consider that from different view angles the observer detects different portions of the top-most and the deeper layers of the canopy [101]. In traditional remote sensing, it is possible to estimate leaf pigment concentrations in the canopy using hyperspectral data. Similarly, using hyperspectral anisotropy data, it might be possible to derive the distribution of pigments within the vertical structure of the canopy.

\subsection{Conclusions}

In this paper we presented a fast and innovative method to capture reflectance anisotropy using a pushbroom spectrometer mounted on a UAV as an alternative for cumbersome goniometer measurements. We used this method to collect the reflectance anisotropy of several agricultural crops at two different growth stages and fitted the RPV model to parameterize and interpret the measurements. Measurements of barley and winter wheat before heading showed very similar anisotropic reflectance patterns that were dominated by backward scattering $(\Theta<$ 0 ) over the measured spectral domain (450-915 nm), with emphasis on the visible wavelengths, combined with a bowl-shaped anisotropy $(k<1)$ in the NIR band for both crops. The development of grain heads on the second measurement day resulted in different composition of the top layers of the barley and winter wheat canopies, which resulted in distinctively different RPV parameter spectra for both crops, indicating a potentially useful signal of grain head development in the multiangular reflectance observations. Measurements of potato showed a strong reduction of backscattering when the potato crops grew from an open row structure to a fully-developed canopy that was almost completely covering the surface. In our data the change in reflectance anisotropy was very distinct for all measured crops, suggesting that anisotropy effects could potentially be used as a signal in operational remote sensing.

Acknowledgements. This research was funded by a grant from the User Support Programme Space Research (GO/12-15) in The Netherlands. The authors would like to thank the Wageningen University \& Research Unmanned Aerial Remote Sensing Facility (UARSF) for providing the UAV and sensor system used in this study. 



\section{Chapter 4}

\section{Mapping reflectance anisotropy of a potato canopy using aerial images acquired with an unmanned aerial vehicle}

This chapter is based on:

Roosjen, P.P.J.; Suomalainen, J.S.; Bartholomeus, H.M.; Kooistra, L.; Clevers, J.G.P.W. (2017). Mapping reflectance anisotropy of a potato canopy using aerial images acquired with an unmanned aerial vehicle. Remote Sensing, 9(5), 417. 


\begin{abstract}
Viewing and illumination geometry has a strong influence on optical measurements of natural surfaces due to their anisotropic reflectance properties. Typically, cameras on-board unmanned aerial vehicles (UAVs) are affected by this because of their relatively large field of view (FOV) and thus large range of viewing angles. In this study, we investigated the magnitude of reflectance anisotropy effects in the 500-900 nm range, captured by a frame camera mounted on a UAV during a standard mapping flight. After orthorectification and georeferencing of the images collected by the camera, we calculated the viewing geometry of all observations of each georeferenced ground pixel, forming a dataset with multi-angular observations. We performed UAV flights on two days during the summer of 2016 over an experimental potato field where different zones in the field received different nitrogen fertilization treatments. These fertilization levels caused variation in potato plant growth and thereby differences in structural properties such as leaf area index (LAI) and canopy cover. We fitted the Rahman-PintyVerstraete (RPV) model through the multi-angular observations of each ground pixel to quantify, interpret, and visualize the anisotropy patterns in our study area. The $\Theta$ parameter of the RPV model, which controls the proportion of forward and backwards scattering, showed strong correlation with canopy cover, where in general an increase in canopy cover resulted in a reduction of backward scattering intensity, indicating that reflectance anisotropy contains information on canopy structure. In this paper, we demonstrated that anisotropy data can be extracted from measurements using a frame camera, collected during a typical UAV mapping flight. Future research will focus on how to use the anisotropy signal as a source of information for estimation of physical vegetation properties.
\end{abstract}




\subsection{Introduction}

In optical remote sensing, the sensors are mostly passive systems and their measured signal therefore depends on the position and orientation of the sensor and the position of the sun relative to the observed surface. Differences in the intensity of the measured signal of natural surfaces as a function of observation and viewing geometry are the result of their anisotropic reflectance behavior. In its idealized form, this is commonly referred to as the Bidirectional Reflectance Distribution Function (BRDF) [4]. Anisotropic reflectance effects are the result of optical and structural properties of the surface. In the case of vegetation these anisotropic reflectance effects can, for example, be the result of the different proportions of shadowed and sunlit parts of the canopy at different observation geometries [6,7], differences in proportion of the background visible at different observation geometries [102], canopy heterogeneity and architecture $[103,104,93]$, and/or the orientation of leaves $[105,106]$. Knowledge of the anisotropic reflectance characteristics of a surface is important for correction and normalization of effects due to viewing and illumination geometry [21,107,11]. In addition, it can be considered an information source. Information on anisotropic reflectance effects has shown to improve classification results $[108,109,78,110]$ or parameter retrieval such as leaf area index (LAI) [73,111], canopy height [74], and canopy clumping $[75,76]$.

Traditionally, anisotropic reflectance characteristics are studied by performing multi-angular reflectance measurements using goniometer setups. Various goniometers have been developed over the past decades to perform these measurements under controlled laboratory conditions $[20,21,15,7]$ or under field conditions [79,23-25,39,27]. Typically, goniometers are ground-based, static devices that allow for accurate control of the positioning of a sensor to measure the reflectance into predefined directions. Measurements by goniometers can sample only small surface areas due to the relatively short distance between target and sensor, making it difficult to capture a large enough representative area of natural heterogenic targets [82]. Moreover, in situ conditions often complicate the deployment of a goniometer setup, for example in poorly accessible terrain, or in snow [112,113], or above water [114].

Unmanned aerial vehicles (UAVs) provide an alternative platform to field goniometer systems for performing multi-angular reflectance measurements $[36,37,115]$. An advantage of UAV-based multi-angular reflectance measurements is that there is no ground-based device needed, which means that the studied surface does not get disturbed. In addition, the sensor-target distance can be larger because no moving arm is needed to position the sensor at a specific spot above the field, which means that surfaces made of larger objects, like forests, can also be studied [90]. Theoretically, the sensor-target distance can even be easily hundreds of meters if such UAV flight altitudes are allowed by local legislation [35].

Recently, several methods and sensors for UAV-based multi-angular sampling have been explored. By using dedicated flight patterns to maneuver a UAV around a target while using a gimbal to point a spectrometer or camera towards the center 
of the target, multi-angular measurements can be acquired [36,37]. By a different strategy, multi-angular observations were obtained by extracting the viewing geometry of measurements by a pushbroom spectrometer mounted on a UAV [115]. By hovering a UAV above fields covered with a single crop and rotating the UAV around its vertical axis, multi-angular measurements were collected within the FOV of the spectrometer. Several other studies have demonstrated how to use a frame camera on-board a UAV to acquire multi-angular measurements $[38,87,116]$. The angular coverage, i.e. the range of azimuth and zenith angles that are sampled, of multi-angular measurement methods relies on the position, orientation, and FOV of the sensor, as well as on the number of samples that are taken. Orthorectification and georeferencing by photogrammetric processing of frame-based camera imagery also relies on multiple views of the same pixel. Thus, by determining the observation geometry of pixels that are captured by multiple cameras, it is possible to collect multi-angular data and thereby location/pixel specific anisotropy information.

In this paper, we used a frame camera to study pixel-wise anisotropy effects based on the overlapping pixels-and therefore multi-angular observations-that are collected during a typical UAV flight for mapping purposes. We define a mapping flight as a flight where several parallel flight lines are flown in a block pattern with sufficient forward and sideways overlap of the collected images for photogrammetric processing to construct a map of the covered area. We present the geometrical equations that allow for the calculation of the observation and illumination geometry of georeferenced ground pixels. In addition, we quantify, visualize, and interpret the anisotropy effects based on the parameters of the semiempirical Rahman-Pinty-Verstraete (RPV) model [66], obtained by fitting the model through our measurements. Moreover, we correlate the RPV parameters with LAI and canopy cover in a potato field where different nitrogen fertilization treatments were applied to sections in the field to study the effect of canopy development on reflectance anisotropy.

\subsection{Materials and methods}

\subsubsection{Study area}

The study area was a field with consumer potatoes (Solanum tuberosum L., cultivar Fontane) of approximately $450 \times 200 \mathrm{~m}$, located south of the village Reusel $\left(51^{\circ} 59^{\prime} 47.9^{\prime \prime} \mathrm{N}, 5^{\circ} 9^{\prime} 34.5^{\prime \prime} \mathrm{E}\right)$ on the Dutch-Belgian border, in the province of NoordBrabant (The Netherlands). The planting date was 16 April 2016. In this field, an experiment was performed to evaluate the effect of split-level fertilization on potato yield. Different initial fertilization levels (before planting) and sensor-based additional sensor-based fertilization (during crop growth) were applied to zones in the field (figure 4.1 and table 4.1). The sensor-based variable-rate fertilization was applied based on crop reflectance measurements performed by Fritzmeier ISARIA crop sensors (Fritzmeier Umwelttechnik, Germany) mounted in front of the tractor providing information on nitrogen need of the crop on a weekly basis. The variable 
rates for nitrogen were based on measured indices from the crop sensor and decision rules derived from previous dedicated fertilization experiments between 2010 and 2013 [117], and applied using a GNSS guided fertilizer spreader. Eight 30 $\times 30$ meter plots (figure 4.1: A-H) were created in the different fertilization zones where weekly measurements of leaf area index (LAI) and leaf chlorophyll content were performed to monitor the status of the potato plants. Sensor-based nitrogen fertilization was applied to half of the plots on 28 June 2016, 15 July 2016, and 9 August 2016. In addition, on 7 July 2016, fertilization with potassium of $60 \mathrm{~kg} / \mathrm{ha}$ was applied to all plots.

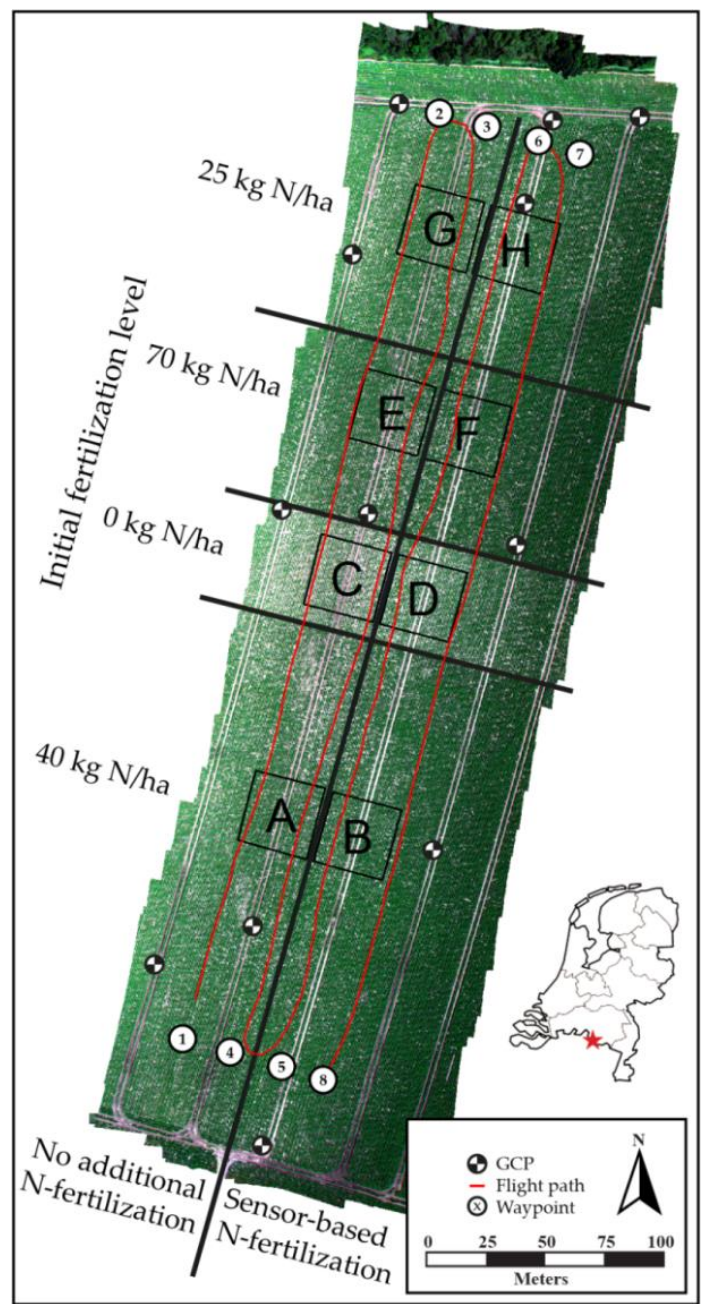

Figure 4.1. The eight experimental plots $(\mathrm{A}-\mathrm{H})$ in the potato field with different initial nitrogen $(\mathrm{N})$ fertilization levels (horizontal zones), and additional nitrogen fertilization levels (vertical zones). The red line indicates the flight path flown during Flight 1 on 9 June 2016 (table 4.2). The base map is an RGB composite ( $\mathrm{R}=674 \mathrm{~nm}$, $\mathrm{G}=559 \mathrm{~nm}$, and $\mathrm{B}=500 \mathrm{~nm}$ ), produced with the data collected during the flight. 
Table 4.1. Overview of the nitrogen $(\mathrm{N})$ fertilization applied to the various plots within the potato experimental field in 2016.

\begin{tabular}{cccc}
\hline Plot & $\begin{array}{c}\text { Initial Fertilization } \\
\text { before Planting } \\
\text { (kg N/ha) }\end{array}$ & $\begin{array}{c}\text { Additional Fertilization } \\
\text { 28 June 2016 } \\
\text { (kg N/ha) }\end{array}$ & $\begin{array}{c}\text { 15 July 2016 } \\
\text { (kg N/ha) }\end{array}$ \\
\hline A & 40 & 0 & 0 \\
B & 40 & 42 & 30 \\
C & 0 & 0 & 0 \\
D & 0 & 140 & 30 \\
E & 70 & 0 & 0 \\
F & 70 & 0 & 49 \\
G & 25 & 0 & 0 \\
H & 25 & 84 & 38 \\
\hline
\end{tabular}

The ground-based apparent LAI (hereafter referred to LAI) was estimated based on weekly measurements with a Cropscan Multispectral Radiometer that used the weighted difference vegetation index (WDVI) [118] for estimating LAI. Calibration curves between the WDVI and LAI were determined by performing both Cropscan measurements and LAI2000 Plant Canopy Analyser measurements in the field at the same farm for potatoes grown in the years 2010-2014 [119]. In 2016, limited measurements with the LAI2000 were available, but validation with these measurements showed estimating LAI with Cropscan data was accurate. The percentage of canopy cover was estimated by classification of the pixels in orthophotos of the study area at high spatial resolution $(\sim 8 \mathrm{~cm}$ ground sampling distance (GSD)) into a vegetation and non-vegetation class. For this, we calculated the normalized difference vegetation index (NDVI) (equation 4.1).

$$
N D V I=\frac{R_{848 \mathrm{~nm}}-0.5\left(R_{658 \mathrm{~nm}}+R_{674 n m}\right)}{R_{848 \mathrm{~nm}}+0.5\left(R_{658 \mathrm{~nm}}+R_{674 n m}\right)}
$$

where $R_{658 n m}, R_{674 n m}$, and $R_{848 n m}$ are the reflectance factors at the subscripted wavelengths. We chose these bands because they are closest to Red Band 4 (665 $\mathrm{nm})$ and NIR Band 8 (842 nm) of the Multi-Spectral Imager (MSI) onboard ESA's Sentinel-2 satellite, that are used for NDVI calculation [120]. The average of Rikola bands at 658 and $674 \mathrm{~nm}$ was calculated to approximate the reflectance of MSI Band 4 at $665 \mathrm{~nm}$. Pixels with an NDVI $>0.7$ were classified as vegetation. We chose this threshold value since it provided good results in separation of the vegetation and non-vegetation classes based on visual inspection.

\subsubsection{UAV flights}

Two UAV flights, with an Altura AT8 octocopter, were performed during the growing season in the summer of 2016 (table 4.2); one flight at an early growing phase ( 9 June 2016) and one flight at a late growing phase after the potato canopy was fully developed (19 July 2016). Between flights on the two dates, additional sensor-based fertilization was given to half of the plots (table 4.1). The flights were performed under clear-sky conditions to assure the strongest anisotropy effects 
[89]. The UAV was programmed to fly at $120 \mathrm{~m}$ above ground level with a horizontal speed of $4 \mathrm{~m} / \mathrm{s}$ from waypoint to waypoint, resulting in four flight-lines that were spaced $20 \mathrm{~m}$ apart (figure 4.1). Images were collected continuously at an interval of $2.4 \mathrm{~s}$. Flying at the aforementioned height and speed with the camera that we used in our study (Section 4.2.3) resulted in forward and sideways overlap of approximately $88 \%$ and $75 \%$, respectively, with a GSD of approximately $8 \mathrm{~cm}$.

Table 4.2. Flights, times, and solar geometry (solar azimuth angle [SAA] and solar zenith angle [SZA]). Time is local time (UTC $+2 \mathrm{~h}$ ). The start time indicates the time at which the first used image was taken and the end time indicates the time at which the last used image was taken.

\begin{tabular}{cccccc}
\hline Flight \# & Date & Start time & End time & SAA $\left[{ }^{\circ}\right]$ & ${\text { SZA }\left[{ }^{\circ}\right]}$ \\
\hline 1 & 9 June 2016 & $12: 18$ & $12: 25$ & $144-147$ & $32-32$ \\
2 & 19 July 2016 & $12: 29$ & $12: 37$ & $147-150$ & $34-33$ \\
\hline
\end{tabular}

\subsubsection{Spectral measurements}

The sensor used for our measurements was a frame-based hyperspectral camera (Rikola Ltd., Oulu, Finland), which has a horizontal and vertical FOV of $36.5^{\circ}$ and collects images of $1010 \times 1010$ pixels. The camera uses the Fabry-Perot interferometer (FPI) technology to capture a series of snapshot images on programmatically selectable spectral bands in the visible-near infrared (VIS-NIR) wavelength range. An FPI system uses an optical filter with two semi-transparent mirror films facing each other. The wavelength of the transmitted light depends on the distance between these two films (the air gap). Thus, by taking a sequence of images while varying the distance between the films, a complete spectrum can be captured for each pixel. As the images on different wavelengths are collected at different times, each wavelength covers a slightly different extent and has a slightly different orientation due to the movement of the UAV during the collection of a full datacube. The number of spectral bands that can be sampled and the center wavelengths of those can be selected according to the user's preference. For our measurements, we sampled 16 bands in the 500-900 nm range (table 4.3). A full datacube of 16 bands in raw format is $32.0 \mathrm{MB}$. The memory card that we used in our configuration, a 32.0 GB SanDisk CompactFlash memory card, has space for potentially storing 999 images of 16 bands.

Prior to take-off for each flight, a dark current (image with lens cap on) measurement and image of a reflectance reference panel (gray 50\% Spectralon panel [LabSphere Inc., North Sutton, NH, USA]) were taken. The HyperspectralImager 2.0 software by Rikola was used to convert the raw images to radiances. The radiance images were then transformed into reflectance factor images using the empirical line method and the measurement of the Spectralon panel. As the images were taken in clear atmospheric conditions from a relatively low altitude, performing advanced atmospheric correction to the images was not found to be necessary. 
Table 4.3. Center wavelengths and Full-Width-at-Half-Maximum (FWHM) of the 16 spectral Rikola bands used in this study.

\begin{tabular}{ccc}
\hline Band & Centre Wavelength [nm] & FWHM [nm] \\
\hline 1 & 500.2 & 14.8 \\
2 & 547.0 & 13.2 \\
3 & 558.8 & 13.0 \\
4 & 568.8 & 12.9 \\
5 & 657.6 & 13.0 \\
6 & 673.6 & 13.2 \\
7 & 705.8 & 13.1 \\
8 & 739.0 & 19.4 \\
9 & 782.8 & 18.5 \\
10 & 791.6 & 18.4 \\
11 & 810.3 & 18.1 \\
12 & 829.0 & 17.8 \\
13 & 847.8 & 17.6 \\
14 & 864.7 & 17.4 \\
15 & 878.7 & 17.3 \\
16 & 894.7 & 17.1 \\
\hline
\end{tabular}

\subsubsection{Orthorectification and measurement geometry}

The reflectance factor images were aligned and ortho- and georectified in PhotoScan Professional 1.2.6 (AgiSoft LLC, St. Petersburg, Russia). Each of the 16 spectral bands had to be processed individually due to the movement of the UAV during the collection of a full datacube [121]. Per measured spectral band, the reflectance factor images were aligned (accuracy setting: highest) using the GPS data collected by the Rikola sensor during the flights as a reference. Thereafter, all bands were merged, and 12 natural ground control points (GCPs) (figure 4.1) were used for accurate georeferencing of the data. The GCPs were manually selected in each of the 16 spectral bands. Unfortunately, there were no dedicated Real Time Kinematic (RTK) or GPS/GNSS ground control markers available at the time of the flights. The imagery was used to calculate and export a Digital Surface Model (DSM) of the target area at a $5 \mathrm{~m}$ ground pixel size in WGS84 UTM 31N coordinate system. Each reflectance factor image was georectified and resampled separately to the same $5 \mathrm{~m}$ ground pixels and exported as separate GeoTIFFs. We specifically chose a $5 \mathrm{~m}$ ground pixel size, because a pixel of this size is large enough to capture a representative proportion of potato rows and exposed soil between the potato rows, while it is small enough to capture the spatial variation caused by the different fertilization treatments applied to the plots in the field. Finally, the photogrammetric positions of the camera $\left(x_{\text {cam }}, y_{\text {cam }}, z_{\text {cam }}\right)$ during each image acquisition were exported as an ASCII file. The PhotoScan processing was performed on a computer with a Windows 7 Enterprise (64 bit) operating system, with an Intel(R) Xeon(R) CPU E5-1650 0 processor at $3.20 \mathrm{GHz}$ (12 CPUs), an AMD FirePro V5900 2 GB graphics card, and $8.00 \mathrm{~GB}$ of installed memory. The processing time of the aforementioned steps was approximately $3.5 \mathrm{~h}$ per flight. 
After the PhotoScan processing, the location of the ground pixels $\left(x_{p x}, y_{p x}, z_{p x}\right)$ were known. Based on the pixel and camera locations, we calculated the View Azimuth Angle (VAA) and View Zenith Angle (VZA) for each ground pixel and reflectance factor image combination. The solar illumination angle for each pixel, relative to the normal of the surface $\left(\beta\left(x_{p x}, y_{p x}\right)\right)$ was calculated using

$$
\begin{gathered}
\cos \beta\left(x_{p x}, y_{p x}\right) \\
=\cos \theta_{s} \cos \theta_{n}\left(x_{p x}, y_{p x}\right)+\sin \theta_{s} \sin \theta_{n}\left(x_{p x}, y_{p x}\right) \cos \left[\varphi_{s}\right. \\
\left.-\varphi_{n}\left(x_{p x}, y_{p x}\right)\right]
\end{gathered}
$$

where $\theta_{s}$ and $\varphi_{s}$ are the sun zenith and sun azimuth angle (table 4.2), respectively. $\theta_{n}$ and $\varphi_{n}$ are the slope and aspect of the pixel, respectively, which were calculated from the DSM. The VZA $\left(\theta_{v}\right)$ was calculated using

$$
\theta_{v}\left(x_{p x}, y_{p x}\right)=\arctan \left(\frac{z_{c a m}-z_{p x}}{\sqrt{\left(x_{c a m}-x_{p x}\right)^{2}+\left(y_{c a m}-y_{p x}\right)^{2}}}\right)
$$

The VAA $\left(\varphi_{v}\right)$ of each pixel was calculated using

$$
\varphi_{v}(x, y)=\arctan \left(\frac{y_{p x}-y_{c a m}}{x_{p x}-x_{c a m}}\right) .
$$

Equation 4.3 assumes that the measured surface is flat. To correct for variations in slope due to height differences in the field, equations 4.3 and 4.4 were substituted in equation 4.2 , resulting in

$$
\begin{aligned}
\cos \theta_{v_{\text {corrected }}}( & \left.x_{p x}, y_{p x}\right) \\
& =\cos \theta_{v}\left(x_{p x}, y_{p x}\right) \cos \theta_{n}\left(x_{p x}, y_{p x}\right) \\
& +\sin \theta_{v}\left(x_{p x}, y_{p x}\right) \sin \theta_{n}\left(x_{p x}, y_{p x}\right) \cos \left[\varphi_{v}\left(x_{p x}, y_{p x}\right)\right. \\
& \left.-\varphi_{n}\left(x_{p x}, y_{p x}\right)\right] .
\end{aligned}
$$

Throughout the rest of this article, $\cos \theta_{v_{\text {corrected }}}$ is referred to as VZA. By applying the equations 4.2-4.5, we were able to obtain the observation and illumination geometry of each individual pixel of each image at each spectral band (figure 4.2). Besides a spatial location and reflectance factor (figure $4.2 \mathrm{~b}$ ), each pixel now has a VAA and VZA (figures 4.2c,d). Pixels that were captured by multiple cameras thus have multiple observation angles as well. 

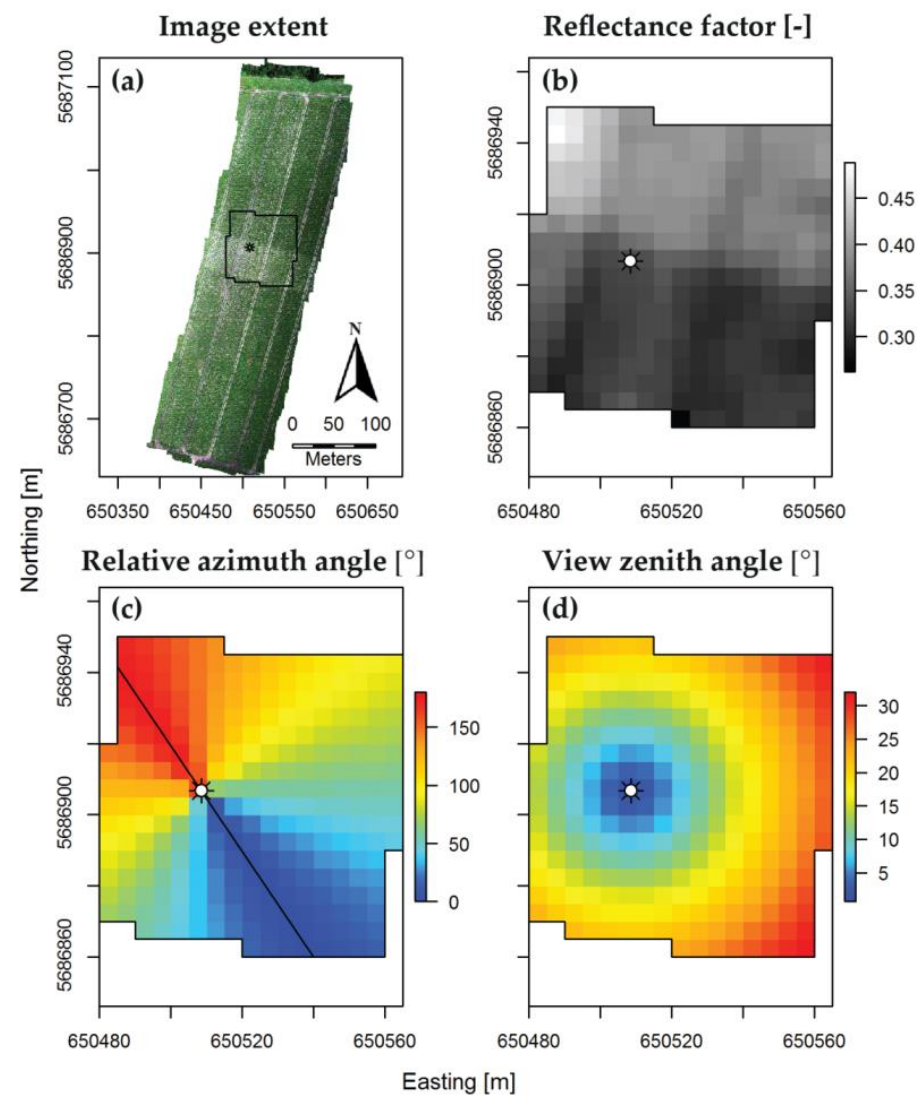

Figure 4.2. An example of the observation geometry of the pixels of one of the georeferenced images. (a) An RGB composite (R=674 nm, G=559 nm, and B $=500$ $\mathrm{nm}$ ) of the study area, collected on 9 June 2016, showing the location of the current image (black line) and the position of the unmanned aerial vehicle (UAV) (sun-shaped symbol); (b) the reflectance factors at $864 \mathrm{~nm}$ of the current image; (c) the relative azimuth angles of the pixels of the current image, where $0^{\circ}$ is the forward scattering direction and $180^{\circ}$ is the backward scattering direction. The solar principal plane is indicated by the black line; (d) the view zenith angle of the pixels of the current image. Projection of a-d is WGS84 UTM 31N.

\subsubsection{Data analysis and visualization}

After determining the observation geometry for all ground pixels and aerial image combinations, we fitted the RPV model [66] to each ground pixel in the study area. The RPV parameters obtained for each pixel were used to create parameterized anisotropy maps of the study area and to analyze the anisotropy patterns that were observed in the different experimental plots. We choose the RPV model because it has been successfully used in a wide variety of anisotropy studies at different spatial scales, ranging from a centimeter scale in laboratory studies [95,77] to a kilometer scale in space-borne studies [122]. The RPV model defines the reflectance 
anisotropy with four parameters where the $\rho_{0}$ parameter controls the amplitude, the $k$ parameter controls the bowl $(k<1)$ / bell $(k>1)$ shape of the anisotropy curve, the $\Theta$ parameter controls forward / backward scattering, and the $\rho_{c}$ parameter controls the hotspot effect around the direct backscattering angle. Mathematically the RPV model is defined as

$$
\rho_{s}\left(\theta_{i}, \varphi_{i} ; \theta_{r}, \varphi_{r}\right)=\rho_{0} \frac{\cos ^{k-1} \theta_{i} \cos ^{k-1} \theta_{r}}{\left(\cos \theta_{i}+\cos \theta_{r}\right)^{1-k}} F(g)[1+R(G)]
$$

where

$$
\begin{gathered}
F(g)=\frac{1-\Theta^{2}}{\left[1+\Theta^{2}-2 \Theta \cos (\pi-g)\right]^{1.5}} \\
g=\cos \theta_{i} \cos \theta_{r}+\sin \theta_{i} \sin \theta_{r} \cos \left(\varphi_{i}-\varphi_{r}\right) \\
1+R(G)=1+\frac{1-\rho_{c}}{1+G} \\
G=\left[\tan ^{2} \theta_{i}+\tan ^{2} \theta_{r}-2 \tan \theta_{i} \tan \theta_{r} \cos \left(\varphi_{i}-\varphi_{r}\right)\right]^{1 / 2}
\end{gathered}
$$

To fit the model to each ground pixel dataset, we applied an iterative optimization method that uses the Levenberg-Marquardt algorithm to minimize the sum of squared residuals (i.e., the difference between the measured reflectance factor at all observation angles and the reflectance factors modeled by the RPV model at the same observation angles) to estimate the parameters. During the fitting procedure, the range of possible solutions for the $\Theta$ parameter was limited between -1 and 1 , the ranges of the $\rho_{0}$ and $k$ parameter were left free. The hotspot parameter $\rho_{c}$ was fixed at 1 , which disables the hot spot parameter, since we acquired no observations close to the hotspot.

\subsection{Results}

\subsubsection{Crop development}

In the data collected on 9 June 2016, the different initial fertilization levels were clearly visible as horizontal zones in NDVI map of the potato field (figure 4.3a). Plot $\mathrm{C}$ and Plot $\mathrm{D}$, the plots in the zone that did not receive any initial fertilization, stood out with clearly lower NDVI values. During the period between the two measurement days, there were two moments at which the potato plants on Plots B, $\mathrm{D}, \mathrm{F}$, and $\mathrm{H}$ received additional sensor-based nitrogen fertilization. This resulted in higher NDVI values in the plots in this section compared to the plots in the section that did not receive any additional fertilization. The effect of the different additional fertilization levels on the NDVI is most clearly visible, as the difference between Plots C and D on 19 July (figure 4.3b), where Plot D has clearly higher NDVI values. 
Figure 4.3c shows the development of the LAI based on Cropscan measurements throughout the growing season.
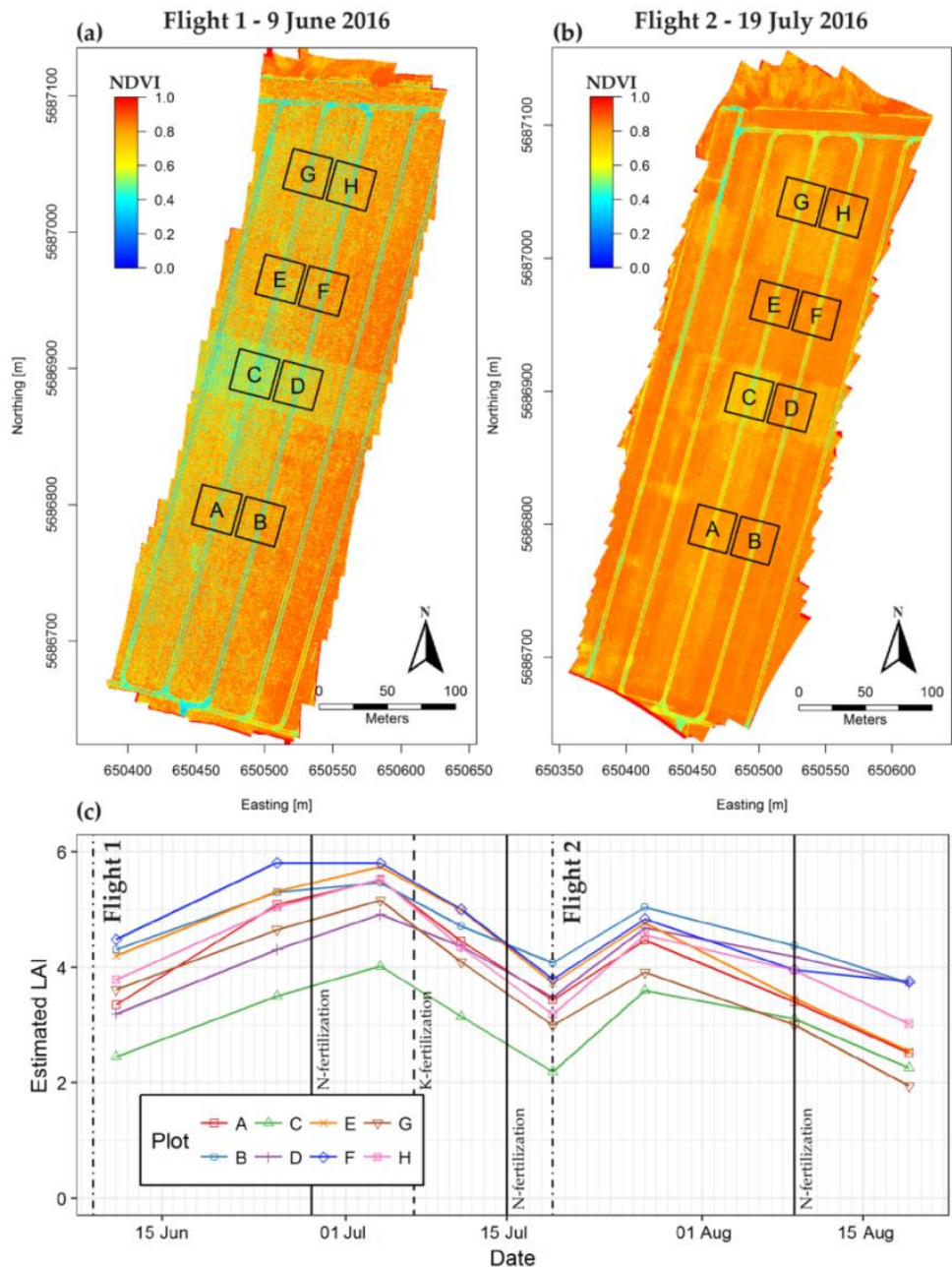

Figure 4.3. Normalized difference vegetation index (NDVI) values of the study area on 9 June 2016 (a) and 19 July 2016 (b), both in WGS84 UTM 31N projection. Estimated leaf area index (LAI) in the experimental plots based on the weighted difference vegetation index (WDVI), calculated from Cropscan measurements (c). The dates at which the additional sensor-based nitrogen $(\mathrm{N})$ fertilization was applied to Plots $\mathrm{B}, \mathrm{D}, \mathrm{F}$, and $\mathrm{H}$ is indicated by the solid lines. The date at which all plots received potassium $(\mathrm{K})$ fertilization is indicated by the dashed line.

During Flight 1 on 9 June 2016, the potato crop was still at an early stage of growth with a haulm (leaves and stems) length of $0.4-0.6 \mathrm{~m}$. The potato plants were standing upright above the ridges, showing a clear row structure. The LAI of all plots increased until the last week of June (figure 4.3c). During the first two weeks 
of July, the LAI decreased, which can be explained by growth-reducing effects caused by a dry period with high temperatures in the area where the study site was located. The insufficient water availability due to the lack of rain, combined with the high temperatures can affect the stomatal capacity of leaves, which in turn can cause a reduction biomass, and thus LAI [123]. Flight 2 on 19 July 2016 occurred after the potato plants were flowered, and the haulm length increased to $0.6-0.9 \mathrm{~m}$. Due to the drought and high temperatures, stems had fallen over and started filling up the space between the potato rows. This resulted in an increase in canopy cover at the date of the second flight, while the LAI remained more or less equal, or for some plots became even lower (table 4.4). After the second flight, the improved water availability due to rainfall and combined with the fertilizer addition of July 15 (table 4.1) resulted in an increase in LAI with the largest long-term effect for the Plots B, D, F and H (figure 4.3c).

Table 4.4. Leaf area index based on Cropscan measurements and the canopy cover during the two measurement days.

\begin{tabular}{ccccc}
\hline & \multicolumn{2}{c}{ 9 June 2016 } & \multicolumn{2}{c}{ 19 July 2016 } \\
\hline Plot & Cropscan [LAI] & Canopy cover [\%] & Cropscan [LAI] & $\begin{array}{c}\text { Canopy cover } \\
\text { [\%] }\end{array}$ \\
\hline A & 3.35 & 61.8 & 3.43 & 91.9 \\
B & 4.31 & 67.7 & 4.08 & 93.8 \\
C & 2.45 & 31.5 & 2.19 & 62.1 \\
D & 3.20 & 46.5 & 3.49 & 91.0 \\
E & 4.20 & 62.5 & 3.73 & 92.8 \\
F & 4.48 & 69.3 & 3.79 & 92.8 \\
G & 3.62 & 61.5 & 2.99 & 88.5 \\
H & 3.79 & 58.7 & 3.19 & 89.8 \\
\hline
\end{tabular}

\subsubsection{View angle coverage}

Based on equations 4.2-4.5, we determined the VAAs and VZAs of each pixel that was georeferenced during the Agisoft PhotoScan processing. Pixels that were captured from multiple camera positions were thus also viewed from multiple observation geometries. As an example, figure 4.4a shows from how many camera positions each pixel in the study area was captured at $658 \mathrm{~nm}$ during the flight on 9 June 2016. An example pixel (the white square in between Plot C and Plot D) was captured from 32 different camera positions in all four flight lines (figure 4.4b).

The nadir normalized reflectance (anisotropy factors (ANIFs) [7]) observed from the different camera positions at $658 \mathrm{~nm}$ for the example pixel indicated a strong anisotropy effect, where the highest ANIFs were observed in the backward scattering direction in the solar principal plane (figure 4.5a). This backward scattering anisotropy was weaker for the same pixel at $848 \mathrm{~nm}$ (figure $4.5 \mathrm{~b}$ ). Due to the movement of the UAV during collection of a full datacube, the camera positions were slightly different for both bands. In addition, the example pixel was not captured from all the same camera positions at both wavelengths. For example, the example pixel was not captured from Camera Positions 12 and 147 at $848 \mathrm{~nm}$. 
On the contrary, the example pixel was not captured from Camera Position 58 at $658 \mathrm{~nm}$, while it was at $848 \mathrm{~nm}$ (compare figures 4.5a and 4.5b).
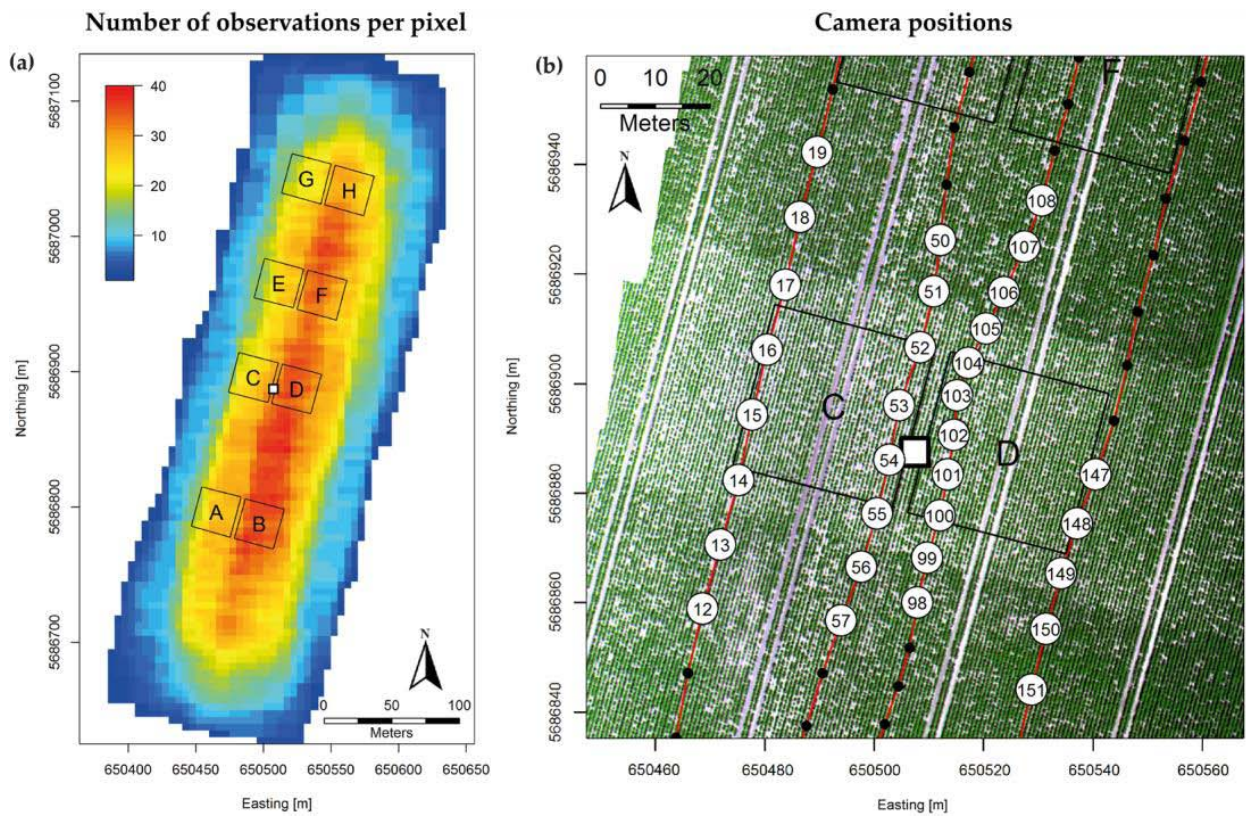

Figure 4.4. The number of observations per pixel, captured during the flight on 9 June 2016, at $658 \mathrm{~nm}$ (a). The camera positions (numbers in white circles) from which an example pixel (white square between Plot C and D) was captured (b). The red lines indicate the flight path of the UAV and the black dots indicate the camera positions that did not capture the example pixel. Both (a) and (b) are in WGS84 UTM $31 \mathrm{~N}$ projection.
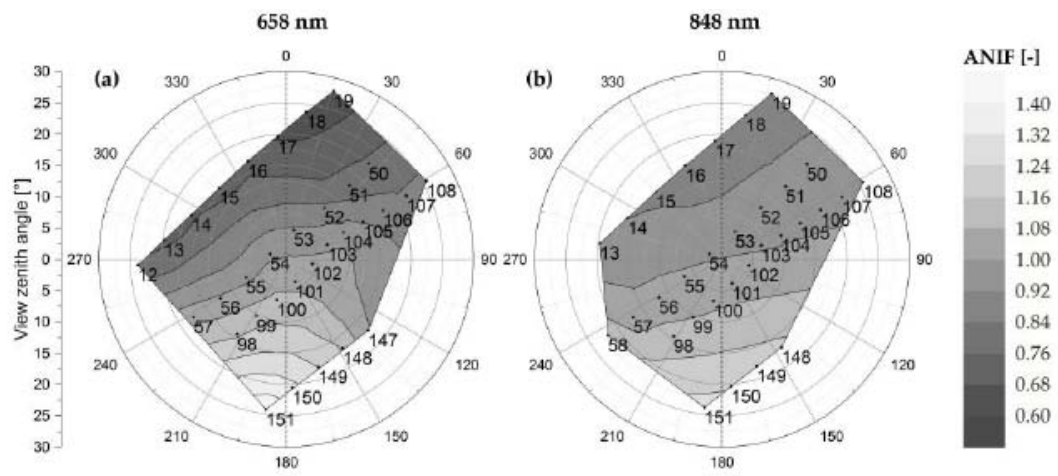

Figure 4.5. Linearly interpolated polar plots of the (anisotropy factors (ANIFs) observed for the example pixel in figure 4.4 at $658 \mathrm{~nm}$ (a) and $848 \mathrm{~nm}$ (b), respectively. The numbers indicate the camera positions and correspond to the numbers in figure $4.4 \mathrm{~b}$. The azimuth angles are relative azimuth angles, where $0^{\circ}$ is the forward scattering direction and $180^{\circ}$ is the backward scattering direction. The vertical dashed line indicates the solar principal plane. 


\subsubsection{Anisotropy maps}

For every georeferenced ground pixel in the study area at each measured wavelength, multi-angular observations as displayed in figure 4.5 were available. By fitting the RPV model through these multi-angular observations at every pixel, we created anisotropy maps that show the spatial distribution of the model parameters.
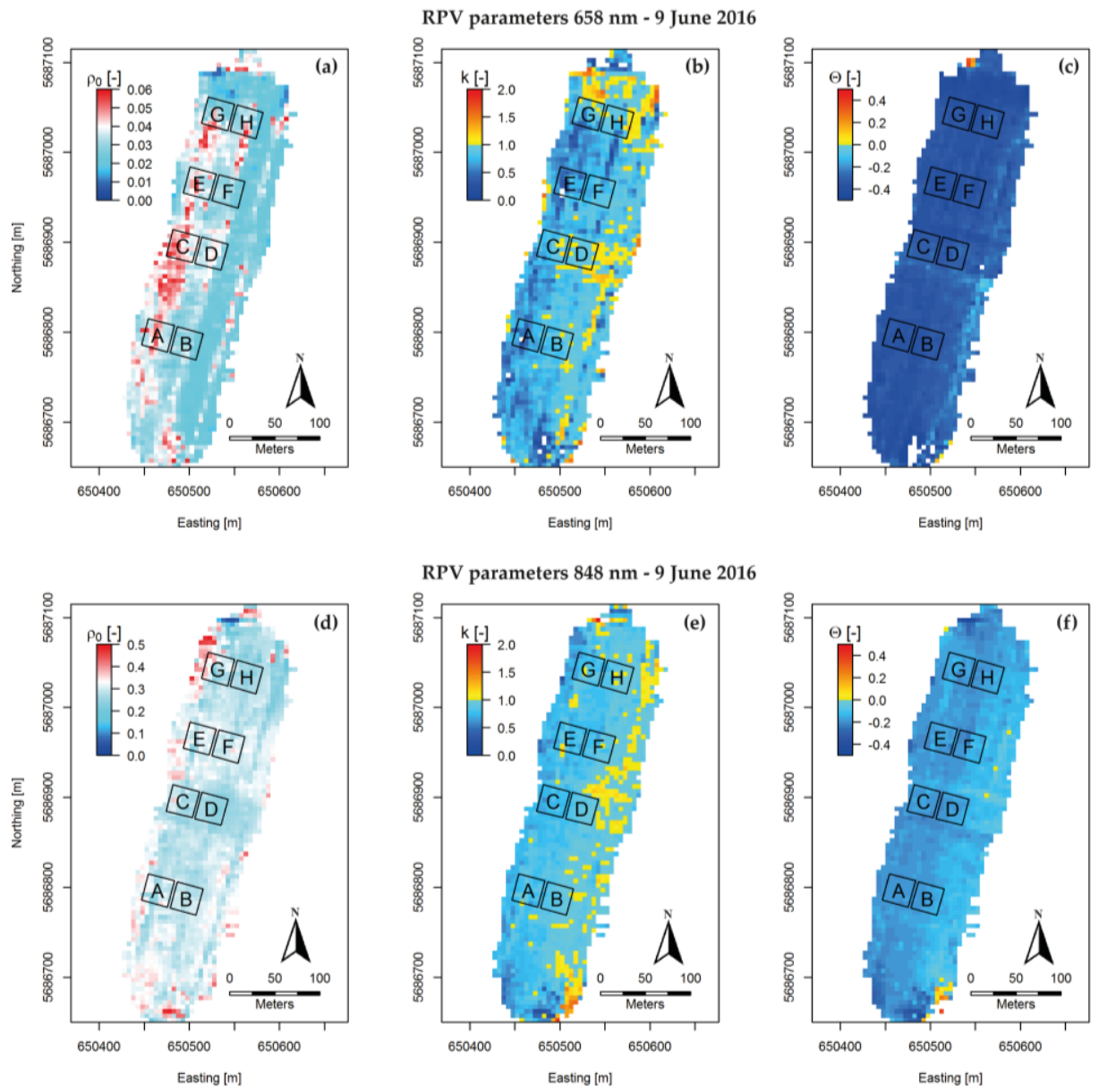

Figure 4.6. $\rho_{0}, k$, and $\Theta$ parameters obtained by fitting the Rahman-Pinty-Verstraete (RPV) model through the measurements of Flight 1 (9 June 2016) at $658 \mathrm{~nm} \mathrm{(a-c)}$ and at $848 \mathrm{~nm}(\mathrm{~d}-\mathrm{f})$. All figures are in WGS84 UTM 31N projection.

Figure 4.6 shows the parameter maps of the $\rho_{0}, k$, and $\Theta$ parameters at $658 \mathrm{~nm}$ and at $848 \mathrm{~nm}$ on 9 June 2016 . The $\rho_{0}$ parameter, which is closely related to the nadir reflectance, follows a typical vegetation trend, with low values at $658 \mathrm{~nm}$ (figure 4.6a) and higher values at $848 \mathrm{~nm}$ (figure $4.6 \mathrm{~d}$ ). Plots $C$ and $D$, which did not receive any initial fertilization and therefore had a lower canopy cover, showed clearly 
lower $\rho_{0}$ values at $848 \mathrm{~nm}$. In general, pixels in both bands had $k<1$, indicating a bowl-shaped anisotropy pattern (Figures 6c,e). Both wavelengths showed clear backward scattering anisotropy $(\Theta<0)$ over the full study area. This backward scattering was particularly strong at $658 \mathrm{~nm}$ (figure 4.6c) and less pronounced at $848 \mathrm{~nm}$ (figure 4.6f).

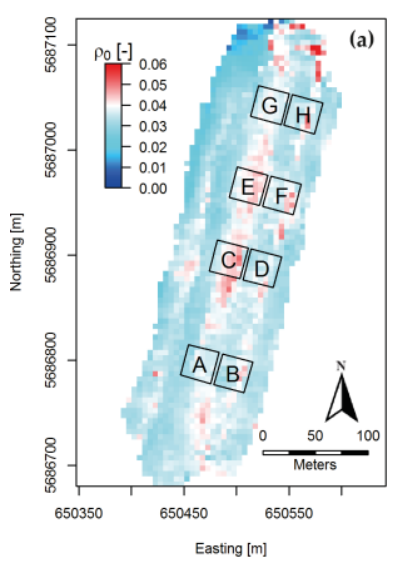

RPV parameters $658 \mathrm{~nm}-19$ July 2016
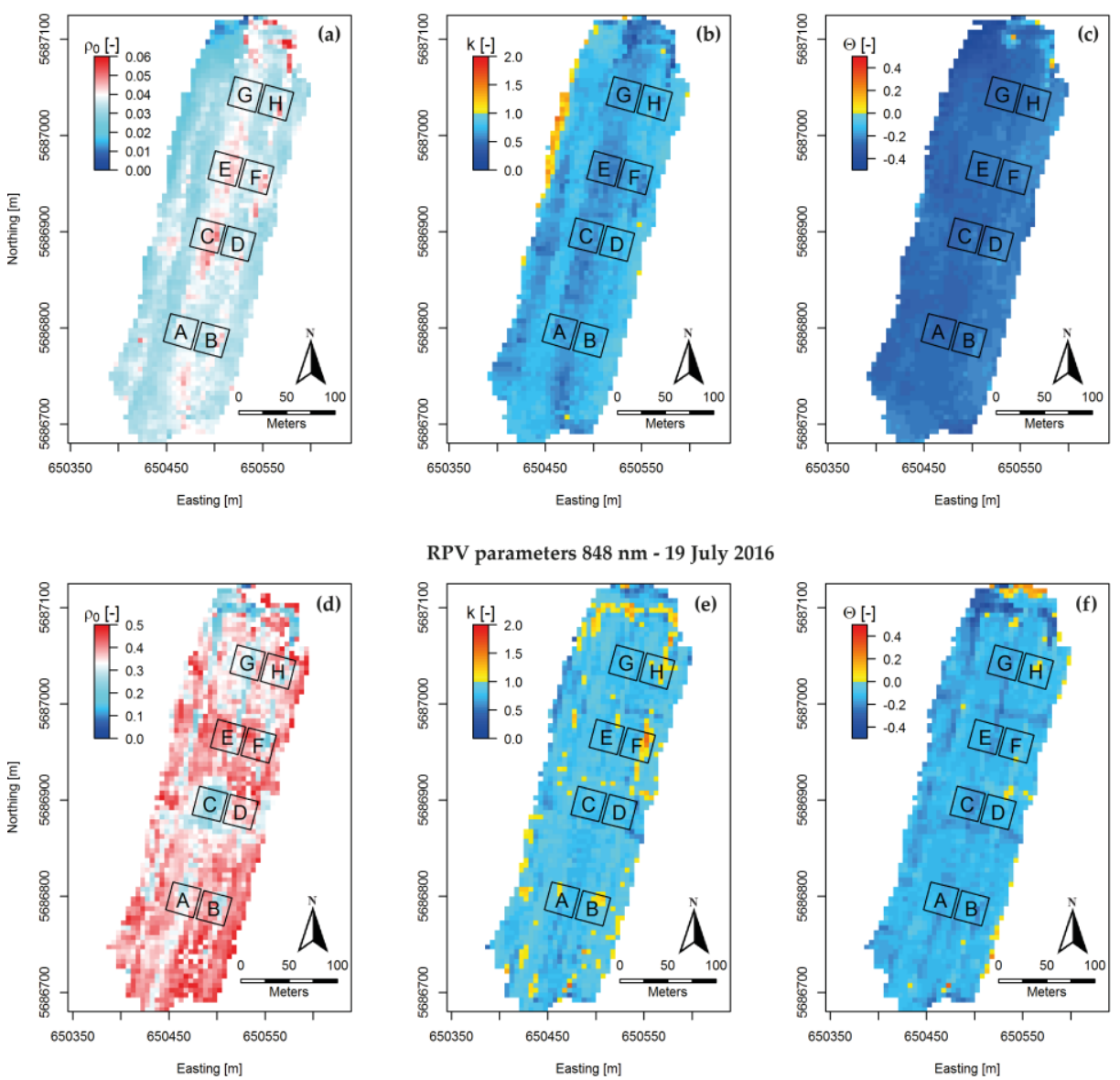

RPV parameters $848 \mathrm{~nm}-19$ July 2016
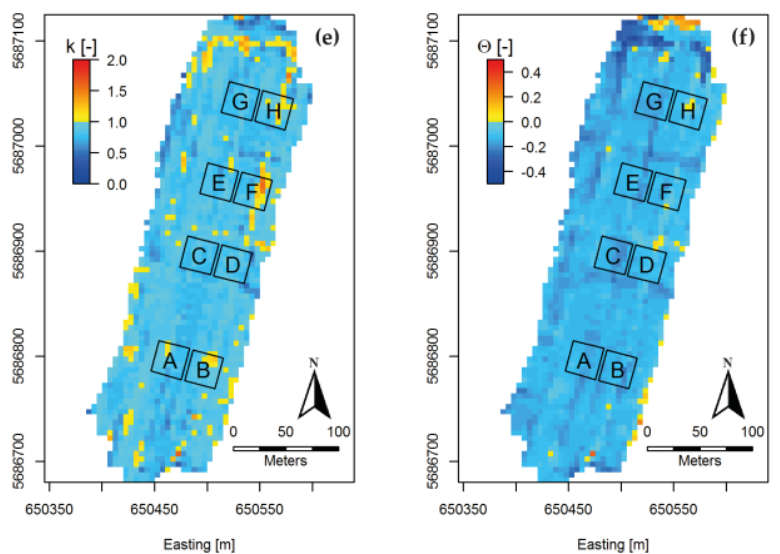

Figure 4.7. $\rho_{0}, k$, and $\Theta$ parameters obtained by fitting the RPV model through the measurements of Flight 2 (19 July 2016) at $658 \mathrm{~nm}(\mathrm{a}-\mathrm{c})$ and at $848 \mathrm{~nm}(\mathrm{~d}-\mathrm{f})$. All figures are in WGS84 UTM 31N projection.

On 19 July 2016, the potato canopy was fully developed, resulting in general in higher $\rho_{0}$ values at $848 \mathrm{~nm}$ (figure $4.7 \mathrm{~d}$ ). The pixels of Plot C, which was the plot in the zone that did not receive any initial fertilization nor additional sensor-based fertilization, had clearly lower $\rho_{0}$ values at $848 \mathrm{~nm}$ than the other plots. The $k$ parameter remained more or less the same as on the first measuring day. The $\Theta$ parameter, on the contrary, showed a strong increase at both wavelengths (i.e., the 
values became less negative), indicating a reduction of backward scattering intensity (compare figure 4.6c,f and figure 4.7c,f).

\subsubsection{Plot statistics}

Figure 4.8 shows the interpolated polar plots of the ANIFs at $658 \mathrm{~nm}$ and $848 \mathrm{~nm}$ for all pixels within Plot $C$ and Plot E, two plots that varied strongly in LAI and canopy cover. Pixels within a $3 \mathrm{~m}$ distance of tractor tracks, which were running through the center of the plots (figure 4.1), were excluded from this analysis.

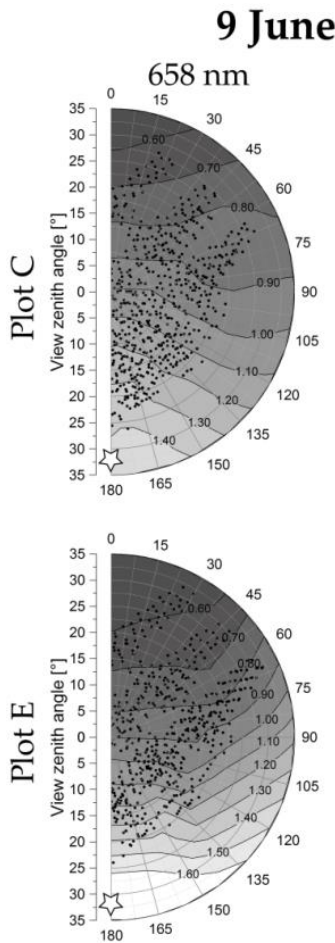

\section{6}
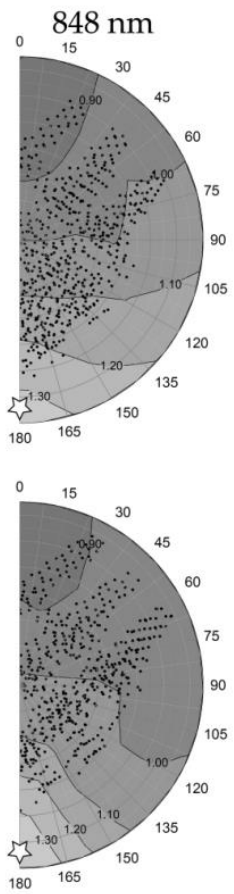

19 July 2016
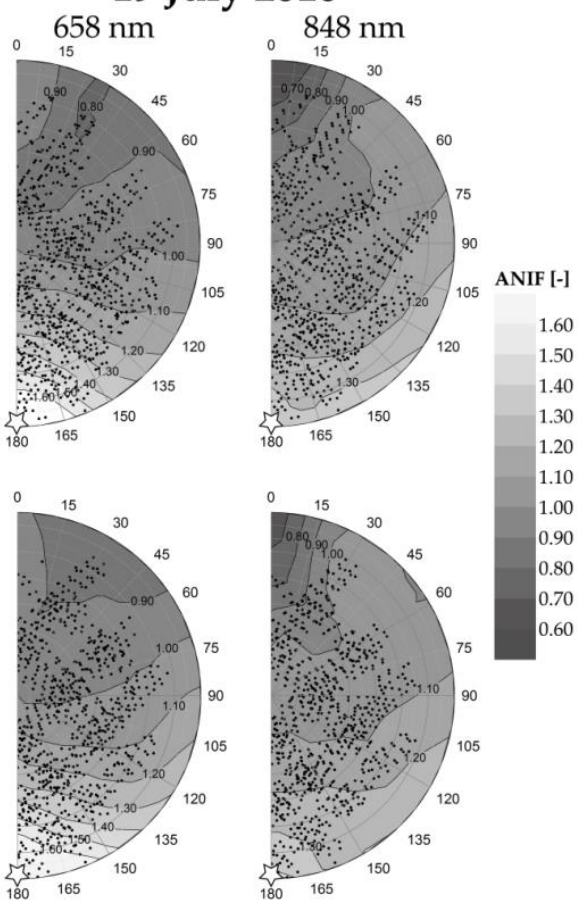

Figure 4.8. . The reflectance anisotropy derived from of all pixels within experimental Plot C (top) and Plot E (bottom) shown as linearly inter- and extrapolated polar graphs at $658 \mathrm{~nm}$ and $848 \mathrm{~nm}$, collected on 9 June 2016 (left) and 19 July 2016 (right), respectively. The white stars indicate the position of the sun during data collection and the black dots indicate the measurement positions.

At both wavelengths, there was a clear backward scattering anisotropy in the plots, which was most pronounced at $658 \mathrm{~nm}$. During the flight on 9 June 2016, we observed a weaker backward scattering anisotropy in Plot C at $658 \mathrm{~nm}$ compared to Plot E, as can be observed by the higher density of isolines. This was likely due to the fact that the potato plant rows in Plot $C$ were not continuous due to gaps in the potato rows, either because of missing plants in the rows or because of the small size of the plants (figure $4.4 \mathrm{~b}$ ). This in turn resulted in fewer strong shadows and 
thereby a weaker backward scattering intensity. The rows of potato plants in Plot $\mathrm{E}$, on the other hand, had fewer gaps and therefore displayed stronger shadows, and thus displayed a stronger backward scattering intensity. During the flight on 19 July 2016, the canopy of Plot E had fully developed into a closed surface with a row structure that was at that point hardly visible. This resulted in strongly reduced row-induced shadow effects and thus decreased backward scattering on this date. On both dates, the backward scattering intensity for both plots at $848 \mathrm{~nm}$ remained more or less similar.
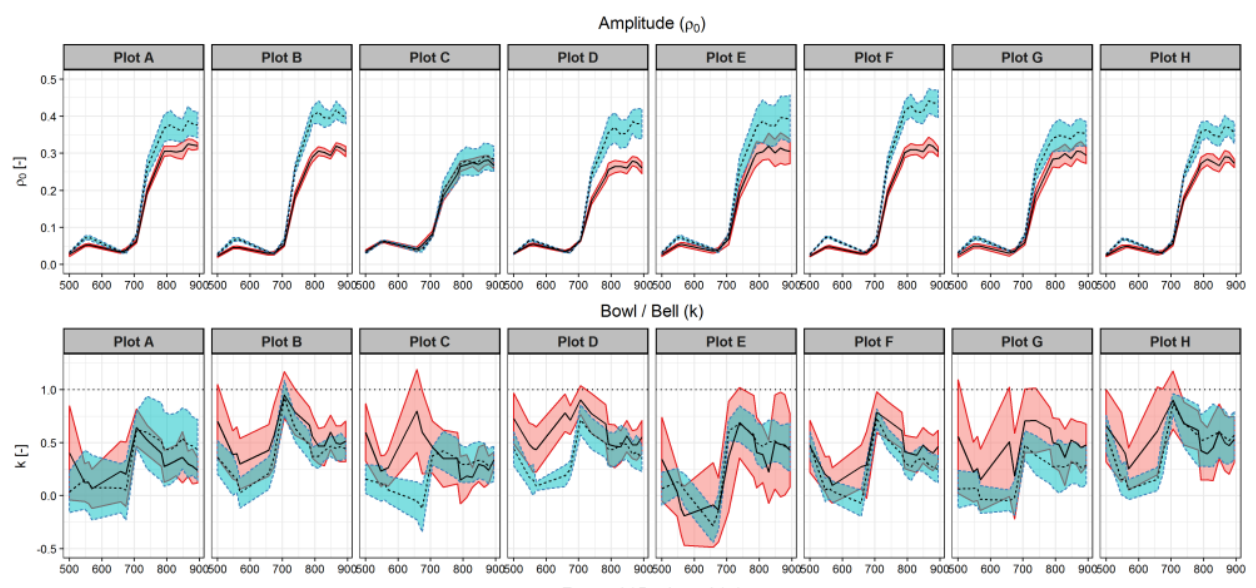

Forward / Backward $(\Theta)$

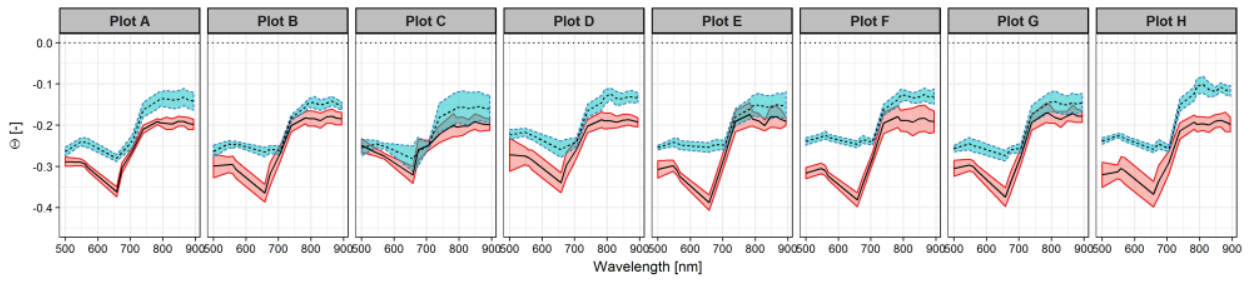

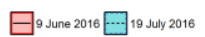

Figure 4.9. Average $\rho_{0}, k$, and $\Theta$ parameters obtained by fitting the RPV model though each individual pixel in the experimental plots on both dates. The colored surfaces indicate the standard deviations.

The average RPV parameters, based on the individually obtained RPV parameter values for every pixel within the experimental plots, are shown in figure 4.9. Again, pixels within a $3 \mathrm{~m}$ radius of the tractor tracks were excluded. The $\rho_{0}$ parameter followed the pattern of a typical vegetation spectrum. In general, a higher $\rho_{0}$ value was observed in the green and NIR wavelength region for plots that had a higher canopy cover on 19 July 2016. This is most clear for the plots where there was a strong increase in canopy cover between the two dates (table 4.4). The $k$ parameter was in general $<1$ at all wavelengths, indicating a bowl-shaped anisotropy pattern for the whole sampled wavelength region. The $\Theta$ parameter, like the $\rho_{0}$ parameter, followed in shape a vegetation spectrum. The strongest backward scattering effects 
(the lowest $\Theta$ values) were observed in the visible wavelength region, with a minimum at $674 \mathrm{~nm}$, where shadow effects are strongest and multiple scattering effects are absent due to the absorption of radiance by chlorophyll in this region. In the NIR region, where shadow effects are diminished due to high reflectance and transmittance by vegetation, the $\Theta$ values were in general less negative than in the visible region. The $\Theta$ values obtained for the flight on Day 2 were consistently higher (less negative) than the $\Theta$ values obtained for the flight on Day 1 , indicating a reduced backward scattering intensity with increased canopy cover.

\subsubsection{RPV parameters vs. canopy cover and LAI}

Figure 4.10 shows the relation between the average RPV parameters of all pixels within each experimental plot and canopy cover at $658 \mathrm{~nm}$ and $848 \mathrm{~nm}$, respectively. On both dates, an increased canopy cover resulted in a decrease in the $\rho_{0}$ parameter at $658 \mathrm{~nm}$ and in an increase at $848 \mathrm{~nm}$. This trend can be explained by the lower reflectance of vegetation at $658 \mathrm{~nm}$ compared to the soil background reflectance at this wavelength. A higher canopy cover therefore resulted in higher reflectance, and thus a higher $\rho_{0}$ value. The opposite holds at $848 \mathrm{~nm}$, where the soil reflectance was lower than the vegetation reflectance. On 9 June 2016 at 658 $\mathrm{nm}$ and on 19 July 2016 at $848 \mathrm{~nm}$, the relations between $\rho_{0}$ and canopy cover were strong, indicated by the $\mathrm{R}^{2}$ of 0.764 and 0.753 , respectively.

The relation between the $k$ parameter and canopy cover was less obvious. On Day 1 at $658 \mathrm{~nm}$, we observed a slight decrease in the $k$ parameter with increased canopy cover $\left(\mathrm{R}^{2}=0.478\right)$. On both dates at $848 \mathrm{~nm}$, the relation between the $k$ parameter and canopy cover was very weak, indicated by the $\mathrm{R}^{2}$ of 0.262 and 0.211 on Day 1 and Day 2, respectively.

On Day 1, we observed a strong decrease in the $\Theta$ parameter (which indicates increased backward scattering intensity) with an increase in the canopy cover at $658 \mathrm{~nm}$. On Day 2, on the contrary, we observed an increase in the $\Theta$ parameter with increasing canopy cover. The decrease in the $\Theta$ parameter on Day 1 happened most likely due to the aforementioned gaps in the potato rows that were present in the plots with low canopy cover on this day: the gaps in the rows result in weaker shadows and thus weaker backward scattering. On Day 2, when there were hardly any gaps left in the rows, we observed a decrease in backward scattering intensity with increasing canopy cover. This is likely due to the disappearance of the rowstructure since the potato rows started growing into each other, narrowing and disappearing the space between the rows, which cause strong shadow effects. 

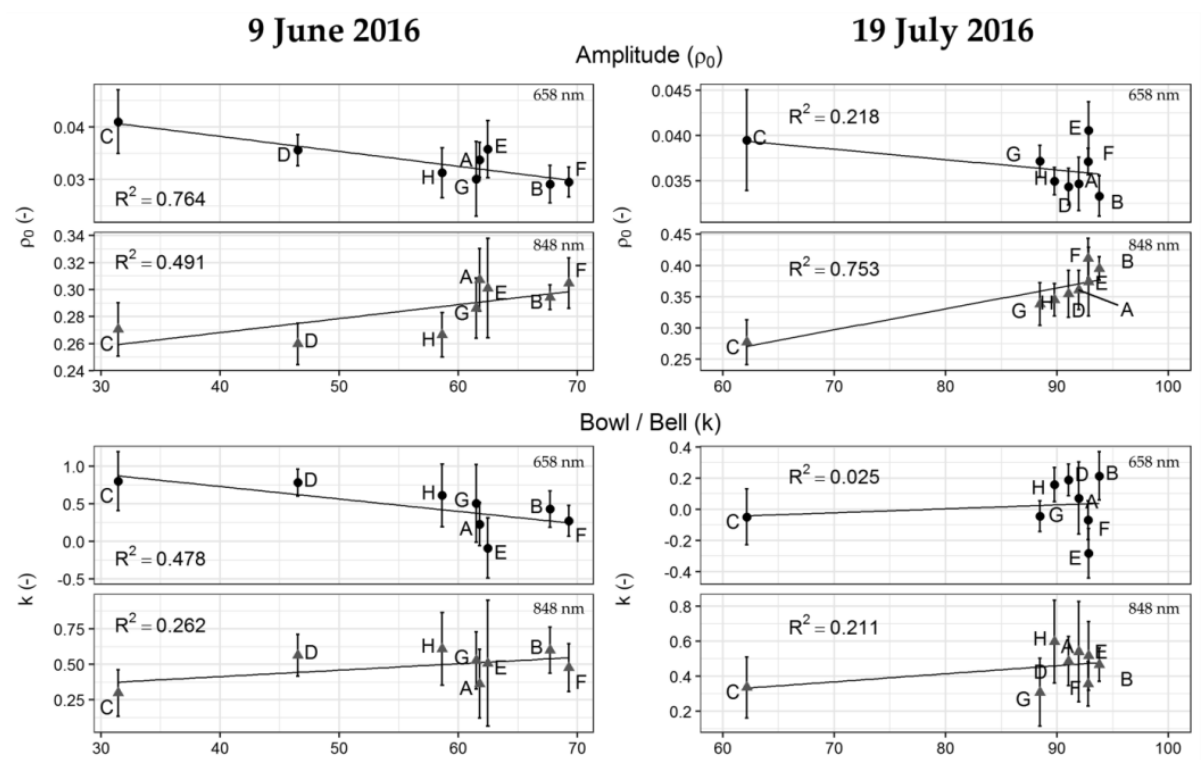

Forward / Backward $(\Theta)$
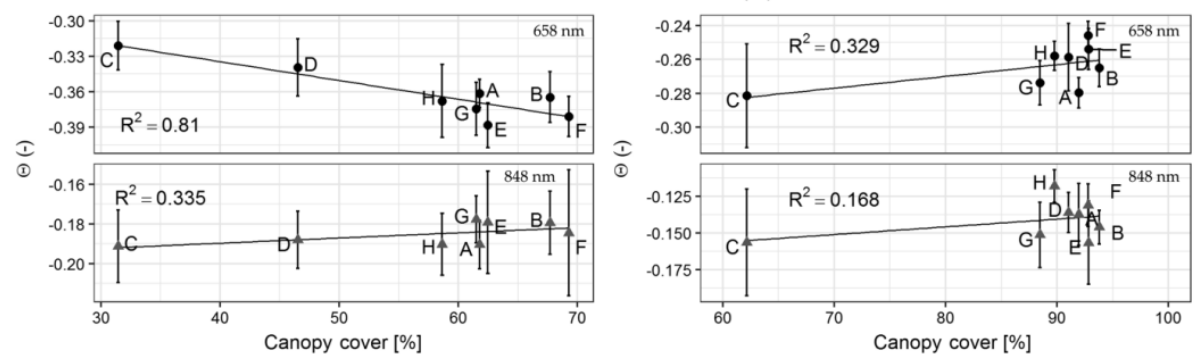

Figure 4.10. The relation between the $\rho_{0}, k$, and $\Theta$ parameters of the RPV model of all pixels in each experimental plot (indicated by letters $\mathrm{A}-\mathrm{H}$ ) and the canopy cover, at $658 \mathrm{~nm}$ (black circles) and $848 \mathrm{~nm}$ (gray triangles), respectively, for both dates. The error bars indicate the standard deviation.

Finally, we studied the correlation of the RPV parameters with the canopy cover and LAI, respectively, for the different experimental plots at all measured spectral bands (figure 4.11). We calculated the Kendall's tau ranking correlation coefficient, since not all canopy cover, LAI, and RPV parameter values were normally distributed, and the number of plots and thus number of observations $(n=8)$ was rather low. The Kendall's tau correlation coefficient takes values between -1 and +1 , where a positive value indicates that the ranks of both variables are increasing and a negative value indicates that the ranks of both variables are decreasing. The closer Kendall's tau gets to +1 or -1 , the stronger the correlation between the variables. Since the flight on Day 1 was performed at an early phase of the potato crop before it reached maximum LAI and the flight on Day 2 at a late phase after reaching maximum LAI, both flights are first analyzed separately and then combined. 
The correlation of the $\rho_{0}$ parameter with canopy cover (figure 4.11a, upper graph) on Day 1 was negative in the visible part of the spectrum, whereas it was positive in the NIR. On Day 2, this correlation was only negative in the red. A significant correlation at $5 \%$ confidence level $(p=0.05)$ was only observed in the NIR for both dates. The pattern for the correlation between $\rho_{0}$ and LAI was quite similar to the one found for canopy cover on both dates (compare figure 4.11b with figure 4.11a, upper graphs).

(a) Canopy cover

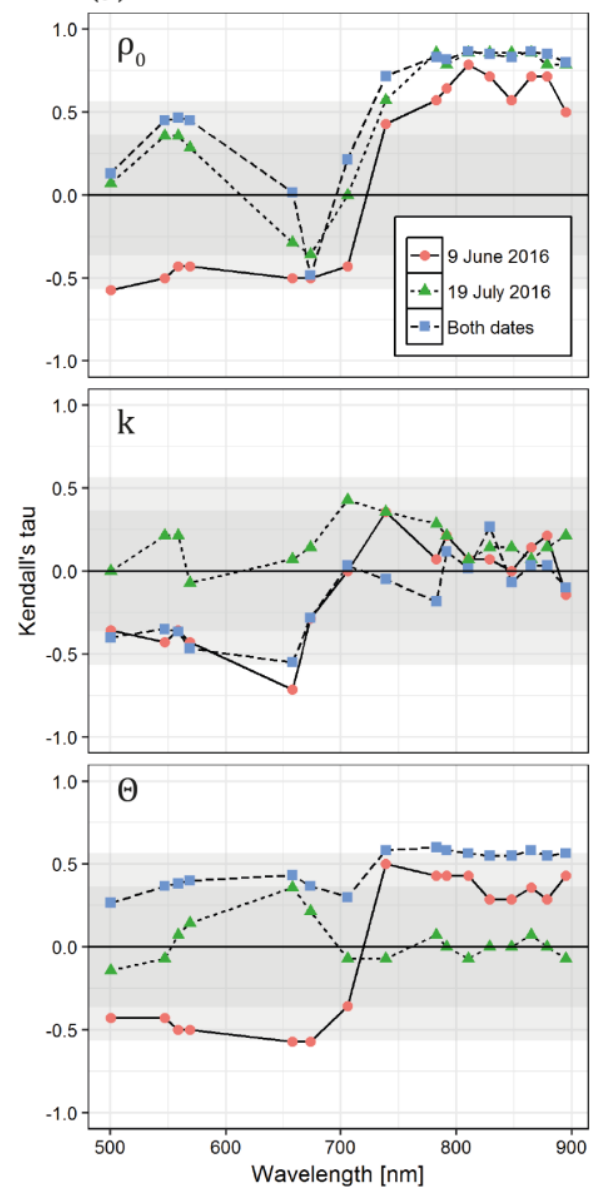

\section{(b) Cropscan LAI}
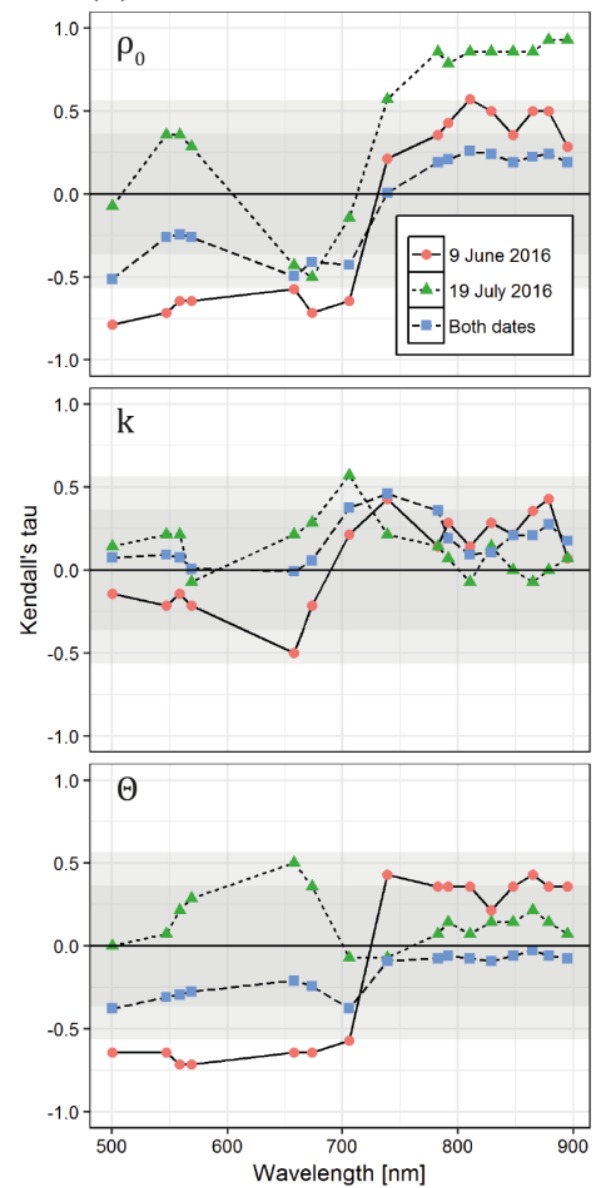

Figure 4.11. Ranking correlation (Kendall's tau) between the $\rho_{0}, k$, and $\Theta$ parameters of the RPV model and the canopy cover (a) and Cropscan LAI (b) for the flights of Day 1 and Day 2 separately and for the flights of both days combined. The gray-shaded areas indicate the significance levels: values above or below the dark-gray areas were significant for the analysis of both dates $(n=16)$ and values above or below the lightgray areas were significant for the analysis of the separate days $(n=8)$, both at the $5 \%$ confidence level. 
The correlation of the $k$ parameter with canopy cover (figure 4.11a, middle graph) was strongest (negative) on Day 1 in the visible part of the spectrum. Only the correlation at $658 \mathrm{~nm}$ was significant. The correlations between the $k$ parameter and LAI (figure 4.11b, middle graph) were quite similar to the one for canopy cover. No correlations were significant at the $5 \%$ level.

The correlation between the $\Theta$ parameter and canopy cover was negative in the visible part of the spectrum and positive in the NIR on Day 1, but they were not significant (figure 4.11a, lower graph). On Day 2, the correlations were close to zero, except for positive, non-significant correlations in the visible part. The correlations between the $\Theta$ parameter and LAI were similar to those of canopy cover. For LAI, the correlations were significant in the visible region on Day 1 (figure 4.11 b, lower graph).

When we combine the data collected on both days, the correlation between the $\rho_{0}$ parameter and canopy cover increased slightly over the whole spectrum (figure $4.11 \mathrm{a}$, upper graph). On the contrary, the correlation between the $\rho_{0}$ parameter and LAI decreased when both days were combined, which indicates that the $\rho_{0}$ parameter was more sensitive to canopy cover than to LAI, and differences between the structure of the potato crop on both dates were not well represented by this parameter. This was likely due to the fact that the LAI did not change much between the two dates, while there was a strong increase in canopy cover (table 4.4). Over the whole spectrum, there was a positive correlation between the $\Theta$ parameter and canopy cover when the data of both dates were combined (figure 4.11a, lower graph), suggesting that in general there was a decrease in backward scattering intensity (increase in the $\Theta$ parameter) when the canopy cover increased.

\subsection{Discussion}

In this paper, we demonstrated how to extract multi-angular observations from overlapping pixels captured by a frame-based camera during a typical UAV mapping flight. After orthorectification and georeferencing of the individual images taken at all measured spectral bands, we were able to calculate the observation geometry of all ground referenced pixels based on the pixel location and camera positions from which the pixels were observed.

During the two flights that we performed during the summer of 2016 over an experimental potato field, pixels were captured in up to 40 different images collected from different positions of the flight path of the UAV (figure 4.4), resulting in up to 40 different observation geometries per pixel. Although the angular sampling coverage of these observations was limited, strong anisotropy effects occurred (figures $4.5 \mathrm{a}, \mathrm{b}$ ). This highlights the importance of taking reflectance anisotropy into account when data is collected at off-nadir viewing angles. Either off-nadir data needs to be corrected and/or normalized to a standardized observation geometry [124], or the exact illumination and observation geometry needs to be considered in further analysis of the data [84]. 
In this study, we sampled a relatively large area, which resulted in a relatively low angular sampling coverage. To achieve a higher sampling density, we suggested focusing on smaller areas and design flight paths with higher overlap and/or to introduce flight lines in multiple azimuth directions covering the area of interest, like for example done by Honkavaara et al. [125] (p. 78, figure 4). Our focus, however, was to derive anisotropy information during a typical UAV mapping flight, and we therefore did not test any alternative flight patterns than the one displayed in figure 4.1. Another option to sample larger VZAs would be to mount the camera tilted under the UAV. However, this could complicate photogrammetric processing steps.

Several other methods to perform multi-angular reflectance measurements of vegetation targets using UAVs have recently been proposed ranging from spectrometers controlled by a gimbal $[36,37]$ to the exploitation of the wide field of view of a hyperspectral line scanner [115]. Using these methods, clear anisotropy effects were observed for different vegetation targets at off-nadir viewing angles, where typically higher reflectance factors were observed in the backward scattering direction, with emphasis on view angles close to the hotspot position $[36,115]$. The drawback of these methods is that the measurements only provide the anisotropy information of a small area or the average anisotropy of a larger surface, respectively. Both of these methods lack information on the spatial distribution of the observed anisotropy effects. The method used in this paper does provide spatially explicit information on anisotropy. By fitting BRDF models, like we did with the RPV model, information on anisotropy can be stored and visualized in maps.

In this study, we used an FPI frame-based camera, which complicates the photogrammetric processing due to the movement of the UAV during the collection of a datacube [121]. In addition, the movement of the UAV during the flight also caused the angular coverage per pixel to be slightly different for each spectral band (figure 4.5). For the fitting of a BRDF model, this does not have to be a problem as long as a large amount of observation angles are available per pixel.

To capture a representative amount of potato rows and spaces between the potato rows in each ground pixel, we resampled the original ground pixels $(\sim 8 \mathrm{~cm})$ to $5 \mathrm{~m}$ ground pixels. For more homogeneous surfaces or surfaces with smaller height differences, a smaller or even the original ground pixel size can be used. The proposed methodology in this study can easily be applied to-which is more commonly available-multispectral camera systems. We used the Rikola camera system because that was the camera we had available. Multispectral cameras, which in general have a higher spatial resolution, allow for analysis at a higher detail level. If local legislation allows it, these cameras can be flown at greater heights above ground level to cover a larger surface area, while maintaining a high spatial resolution. Multispectral camera systems with bands in both the visible and NIR capture the most pronounced changes in anisotropy that relate to increased canopy cover in a potato crop, as can be seen in the $\Theta$ and $\rho_{0}$ parameter spectra in Figure 4.9 and the correlation spectra in figure 4.11. 
We studied the magnitude of anisotropic reflectance effects by analyzing anisotropy parameters obtained by fitting the RPV model through the multiangular observations of each georeferenced ground pixel. Observed trends were a backward scattering anisotropy for the whole study area, which was most pronounced in the visible wavelength region and less pronounced in the NIR region, which is typical for vegetation targets $[6,7]$.

In addition, we studied the effects of canopy cover and LAI on reflectance anisotropy. On the first measuring day, we observed an increase in backward scattering intensity with increased canopy cover at visible wavelengths. This might be related to the higher amount of gaps in the potato rows on this day, which in turn resulted in weaker shadows and thus weaker backward scattering intensity. A higher canopy cover on this day occurred at plots where the potato rows had fewer gaps, which resulted in stronger shadow effects. On the second measuring day, when there were fewer gaps between rows, we observed a decrease in backward scattering intensity with an increase in canopy cover. This decrease is likely the result of the filling up of space between consecutive rows due to potato plants growing into each other (the potato plants had fallen over due to long and unstable haulms in combination with unfavorable weather conditions), which resulted in a decrease in shadows created by the rows. A decrease in backward scattering intensity with canopy closure was observed in a previous UAV-based anisotropy study of potato canopy development [115].

This study demonstrates that pixel-wise multi-angular observations can be obtained from frame-based cameras by calculation of the VAAs and VZAs of georeferenced ground pixels that were captured from multiple camera positions. The information contained in these multi-angular observations is valuable for correction of anisotropic reflectance effects, but also provides additional information to spectral data on vegetation characteristics. Moreover, knowing the observation geometry of every pixel makes it possible to select and analyze only observations that were taken from, for example, the backward scattering direction. In this direction, observations have been demonstrated to be more useful for LAI prediction [126]. Besides this, in the backward scattering direction, shadows are minimized and observations are more sensitive to vegetation pigments [127], which makes frame-based measurements in combination with their observation geometry also interesting for the study of biochemical vegetation parameters.

\subsection{Conclusions}

The overlap of images that are collected during a typical UAV mapping flight using a frame camera provides multi-angular views of georeferenced ground pixels. In this paper, we describe how to extract the observation geometry of these multiangular views. The results of this paper demonstrate that, in clear sky illumination conditions, strong anisotropy effects occur in measurements with off-nadir viewing when using a frame camera. 
By fitting the RPV model through the multi-angular measurements, we parameterized and interpreted the anisotropic reflectance effects captured during two UAV flights in the growing season of 2016 for a potato field that contained eight experimental plots with different LAI and canopy cover levels. Analysis of the anisotropy patterns indicated that in general the potato crop had a higher reflectance in the backward scattering direction. An increase in canopy cover during the growing season resulted in a reduction of this backward scattering, which can be attributed to the diminishing row structure with increasing canopy cover, and thereby weaker row-induced shadow effects.

The results of this study indicate that the parameters describing the anisotropy with the RPV model contain information on structural characteristics of the potato crop such as LAI and canopy cover. This suggests that including such anisotropy information may lead to more accurate estimates of these characteristics. Future research will focus on how to use this anisotropy information to better estimate vegetation parameters, for example, by using radiative transfer models. Moreover, since the method described in this paper can in theory be used to study the anisotropy of any surface, we will apply it to other vegetation, crop, and soil targets as well. Special focus will be on the influence of canopy development on the anisotropy signal.

Acknowledgements: This research was funded by a grant from the User Support Programme Space Research (GO/12-15) in The Netherlands. The authors would like to thank the Wageningen University \& Research Unmanned Aerial Remote Sensing Facility (UARSF) for providing the UAV and sensor system used in this study. In addition, the authors thank Jacob van den Borne for the preparation of and access to the experimental field and Marnix van den Brande for doing the LAI measurements. 



\section{Chapter 5}

\section{Improved estimation of leaf area index and leaf chlorophyll content using multi-angle spectral data collected by an unmanned aerial vehicle}

This chapter is based on:

Roosjen, P.P.J.; Brede, B.; Suomalainen, J.S.; Bartholomeus, H.M.; Kooistra, L.; Clevers, J.G.P.W. Improved estimation of leaf area index and leaf chlorophyll content using multi-angle spectral data collected by an unmanned aerial vehicle. Under review at the International Journal of Applied Earth Observation and Geoinformation. 


\begin{abstract}
In addition to single-angle reflectance data, multi-angular observations can be used as an additional information source for the retrieval of properties of an observed target surface. In this paper, we studied the potential of multi-angular reflectance data for the improvement of leaf area index (LAI) and leaf chlorophyll content (LCC) estimation by numerical inversion of the PROSAIL model. The potential for improvement of LAI and LCC was evaluated for both measured data and simulated data. The measured data was collected on 19 July 2016 by a frame-camera mounted on an unmanned aerial vehicle (UAV) over a potato field, where eight experimental plots of $30 \times 30 \mathrm{~m}$ were designed with different fertilization levels. Simultaneously to the UAV flight, in situ measurements of LAI and LCC were performed. Inversion of the PROSAIL model was done based on nadir data and based on multi-angular data collected by the UAV. Inversion based on the multi-angular data performed slightly better than inversion based on nadir data, indicated by the decrease in RMSE from 0.70 to $0.65 \mathrm{~m}^{2} / \mathrm{m}^{2}$ for the estimation of LAI, and from 17.35 to 17.29 $\mu \mathrm{g} / \mathrm{cm}^{2}$ for the estimation of LCC, when nadir data were used and when multiangular data were used, respectively. In addition, we simulated several datasets at different angular configurations and compared the accuracy of the inversions of these datasets with the inversion based on nadir data. In general, the results indicated that when more viewing angles, more well distributed viewing angles, and viewing angles up to larger zenith angles were available for inversion, the most accurate estimations were obtained. However, when using a realistic angular sampling configuration as covered by the UAV platform, meaning a range of view zenith angles up to $30^{\circ}$, already a huge improvement could be obtained in comparison to nadir viewing. Based on the eight plots in this study, the RMSE of LAI improved from 0.64 to $0.08 \mathrm{~m}^{2} / \mathrm{m}^{2}$, whereas the RMSE of LCC improved from 24.37 to $2.43 \mu \mathrm{g} / \mathrm{cm}^{2}$. The results of this study show that the estimation of LAI and LCC by numerical inversion of the PROSAIL model can be improved when multi-angular observations are introduced.
\end{abstract}




\subsection{Introduction}

Accurate quantitative estimates of vegetation bio-physical/-chemical parameters are important for, e.g., precision agriculture [19], crop phenotyping and monitoring crop traits $[128,129]$, and reduction of fertilizer usage and improvement of yield prediction $[130,131]$. Inversion of radiative transfer models (RTMs) using optical remote sensing data is a commonly applied technique for the estimation of these parameters. Based on physical laws, RTMs describe how radiation interacts with vegetation canopies. RTMs allow for the computation of reflectance at arbitrary viewing and illumination geometries and a set of leaf and canopy parameters. With different inversion schemes, RTMs can be used to estimate these parameters based on a reflectance input. The accuracy of the parameter estimations depends on the used model, the applied inversion technique and the quality of the input data [132]. PROSAIL [127] - a combination of the PROSPECT leaf model [133] and the SAIL canopy bidirectional reflectance model [134], is among the most widely used RTMs. By inversion of the PROSAIL model, it is possible to simultaneously estimate both leaf and canopy parameters such as leaf area index (LAI) and leaf chlorophyll content (LCC) [135].

The inversion of RTMs is an ill-posed problem due to measurement and model uncertainties [136], and due to the issue that different combinations of parameters may result in similar reflectance spectra $[137,138]$. Therefore, inversion of a single spectrum may result in a range of possible parameter combinations that approximate a similar spectral signature. To reduce the number of possible solutions to the inversion of a RTM model, regularization needs to be applied. This can for example be done by constraining parameters, or by the use of a priori information on the parameters $[139,140]$. Another way to improve the estimation of vegetation parameters is by increasing the dimensionality of the input data by introducing multi-angular observations [141-144]. It has been widely accepted that multi-angular reflectance data contain additional information of vegetation targets compared to data at a single observation angle [145]. Multi-angular measurements are sensitive to vegetation structure and can therefore provide enhanced information on the structural properties [146,93]. For example, it has been demonstrated that the retrieval of LAI by inversion of the PROSAIL model for various crops can be improved by inclusion of just a second observation angle for the inversion [88]. Moreover, using multiple viewing angles from satellites has also been demonstrated to improve the simultaneous estimation of LAI and LCC [141].

Some satellite-borne sensors have been specifically designed to make multi-angular observations, such as the Compact High Resolution Imaging Spectrometer on-board the Project for On-Board Autonomy (CHRIS-PROBA) [17] or the Multi-angle Imaging Spectrometer (MISR) [16]. These sensors are capable of viewing an area of interest from several different angles during their overpass, providing information on the anisotropic characteristics of the target's reflectance. Although these sensors are capable of collecting data up to large view zenith angles (VZAs) [five viewing angles up to a VZA of $\pm 55^{\circ}$ for CHRIS and nine viewing angles up to a VZA $\pm 70.5^{\circ}$ for MISR], their relative coarse spatial resolution (18 - 36 m for CHRIS and $275 \mathrm{~m}-1.1$ $\mathrm{km}$ for MISR) hinders detailed analysis for, e.g., precision agriculture. 
In addition to satellite-based measurements, multi-angular observations are typically collected using goniometers. On the one hand, goniometers provide a high angular sampling in general with accurate control of the observation angles $[33,147]$. Moreover, depending on the sensor mounted on the goniometer, measurements with high spectral resolution can be acquired. On the other hand, goniometer measurements are relatively time consuming. Especially for measurements in the field this can be a serious issue due to several factors including movement of the sun and possible changes in atmospheric conditions during a measurement sequence [82]. Moreover, most goniometer systems only sample a single point and thus lack information on the spatial distribution of anisotropy effects.

Recently, multi-angular measurements have also been performed using unmanned aerial vehicles (UAVs). Several researchers have shown that these agile platforms are capable of collecting multi-angular observations in a relatively short amount of time $[36,37,115,148]$. Moreover, due to the relatively low flight height of UAVs, a good ground sampling distance (GSD) can be achieved, making UAV-based methods valuable for detailed studies on anisotropy effects at the individual plant level [148]. UAV-based methods where a gimbal is used to hold a spectrometer at different viewing angles can provide a good angular coverage of an area of interest $[36,37]$. However, to capture viewing angles covering all azimuth directions around a target requires complicated flight patterns, making it difficult to apply such a measurement strategy. Moreover, most professional UAVs have downward pointing sensors and do not have the flexibility to change their orientation during the flight.

In a recent study we demonstrated that multi-angular views can easily be obtained by exploiting the overlap of images that are collected by a frame-camera mounted on a UAV [148]. This method is very attractive for studying pixel wise anisotropy effects, since every georeferenced pixel is captured from multiple camera positions and thus observed from different viewing angles. Using this method it was shown that different fertilization regimes within a potato field resulted in significantly different anisotropy signals due to variation in the development of the potato plants as a result of the differences in fertilization level. In that study, these differences in anisotropy were quantified using the parameters of the Rahman-Pinty-Verstraete (RPV) model [66]. A correlation between RPV and canopy parameters pointed at the information content of the anisotropy signal. The logical follow-up step would be to explore the use of these multi-angular views in combination with physically based RTMs to explore their use for the estimation of vegetation parameters.

The aim of this paper is to study the use of multi-angular measurements for the retrieval of LAI and leaf LCC by inversion of the PROSAIL model. We studied three cases: In the first case we used the data that were collected during the study of Roosjen et al. [148] for inversion of the PROSAIL model to estimate LAI and LCC, which were simultaneously measured in the field during the UAV flight. However, a mismatch between the measured multi-angular reflectance data and the ability of the used model to reproduce this, which can be either caused by the quality of the RTM model or by the quality of the spectral measurements, might provide poor 
results [141]. Therefore, in a second case, we used the configuration of viewing angles that was captured during the same study and simulated reflectance data at these angles, which we then used as input for the inversion of the PROSAIL model to estimate LAI and LCC. This case represents the situation where a realistic range and distribution of viewing angles is available for inversion, and where we were sure that the model used can reproduce the input data. In the third case, we simulated multi-angular reflectance data for inversion of the PROSAIL model based on several configurations of viewing angles that are typically collected with goniometer setups. This case represents the ideal situation where a wide range of well-distributed viewing angles is available. For all cases, the inversion accuracies based on the multi-angular data were compared with inversions based on nadir viewing data, in order to assess the improvement of LAI and LCC estimations when multi-angular observations were used. 


\subsection{Methods}

\subsubsection{Study area}

The study area that was used for our UAV experiment and data simulations, was a potato field (Solanum tuberosum L., cultivar Fontane. Planting date: 16 April 2016) located south of the village Reusel $\left(51^{\circ} 59^{\prime} 47.9^{\prime \prime} \mathrm{N}, 5^{\circ} 9^{\prime} 34.5^{\prime \prime} \mathrm{E}\right)$ on the Dutch-Belgian border, in the province of Noord-Brabant (The Netherlands). In this field, an experiment was performed to evaluate the effect of split-level fertilization on potato yield. Eight $30 \times 30$ meter plots, labelled $\mathrm{A}-\mathrm{H}$, were marked in the different fertilization zones (figure 5.1).

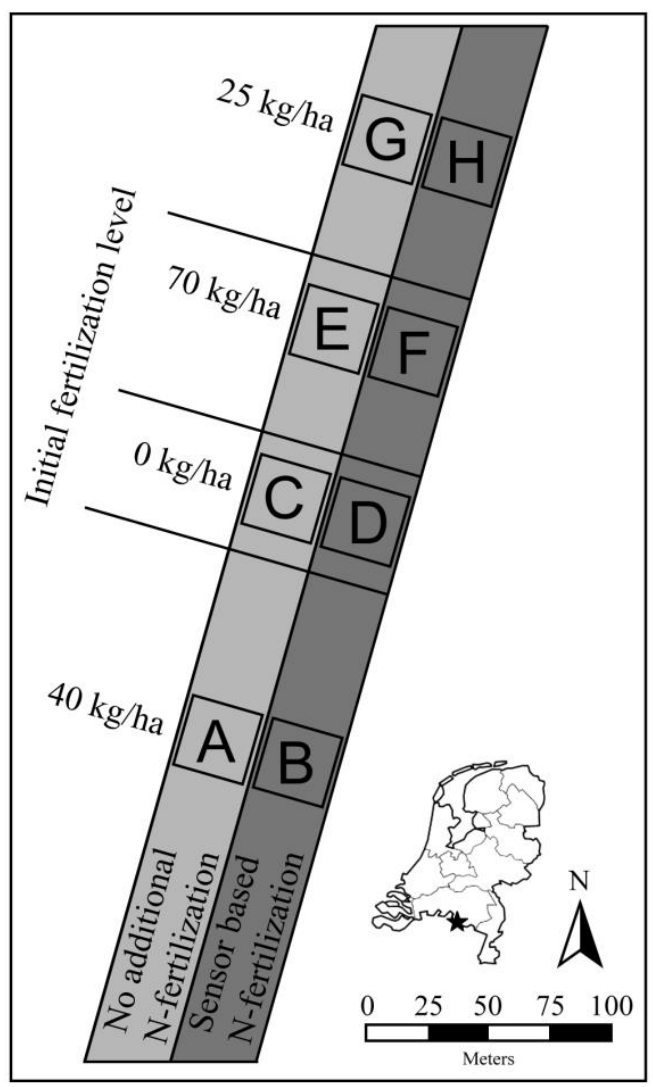

Figure 5.1. A schematic layout of the experimental plots $(A-H)$ in the potato field with different initial nitrogen $(\mathrm{N})$ fertilization levels (horizontal zones), and additional nitrogen fertilization levels (vertical zones).

Varying initial nitrogen $(\mathrm{N})$ fertilization was applied to half of the plots before planting. During the growing season plots B, D, F, and H received additional sensorbased nitrogen fertilization based on reflectance measurements performed by Fritzmeier ISARIA crop sensors (Fritzmeier Umwelttechnik, Großhelfendorf, 
Germany) that were mounted on the front of a tractor, providing information on nitrogen need of the potato plants on a bi-weekly basis. The different fertilization regimes in the field resulted in variation of LAI and LCC in the experimental plots (table 5.1) at the time of data collection (19 July 2016) with the UAV. Measurements of LAI and LCC were performed in situ on the same day as the UAV flight. LAI and LCC were derived from reflectance measurements using a Cropscan Multispectral Radiometer [149]. For a more detailed description of the study area and the fertilizer-experiment, the reader is referred to [149] and [148].

Table 5.1. Leaf area index (LAI) and leaf chlorophyll content (LCC) measured in the experimental plots on 19 July 2016.

\begin{tabular}{ccc}
\hline Plot & LAI $\left[\mathbf{m}^{\mathbf{2}} / \mathbf{m}^{\mathbf{2}}\right]$ & $\mathbf{L C C}\left[\boldsymbol{\mu g} / \mathbf{c m}^{\mathbf{2}}\right]$ \\
\hline A & 3.43 & 39.55 \\
B & 4.08 & 51.37 \\
C & 2.19 & 50.05 \\
D & 3.49 & 65.61 \\
E & 3.73 & 42.46 \\
F & 3.79 & 49.46 \\
G & 2.99 & 41.76 \\
H & 3.19 & 54.87 \\
\hline
\end{tabular}

\subsubsection{Rikola sensor}

Spectral data were collected by a hyperspectral camera (Rikola Ltd., Oulu, Finland), mounted on an Altura AT8 octocopter. The camera collects images of $1010 \times 1010$ pixels and has a horizontal and vertical field of view (FOV) of $36.5^{\circ}$. The camera uses Fabry-Perot interferometer (FPI) technology to capture images at a programmatically selectable set of spectral bands in the visible-near infrared (VISNIR) wavelength range. The sampled wavelength is selected using a piezo driven FPI filter at which the bandpass wavelength depends on the distance (the air gap) between two semi-transparent mirror films. A sequence of wavelengths is collected by varying this air gap, which causes a small time difference between recording of each spectral band in a collected image. The bands that were sampled are shown in table 5.2. For a detailed description of the radiometric calibration of the collected images, the reader is referred to [148].

\subsubsection{UAV viewing angles}

Spectral data were collected in clear atmospheric conditions during four back-andforth flight lines that were spaced $20 \mathrm{~m}$ apart, covering the experimental plots in the potato field. The UAV was programmed to fly at $120 \mathrm{~m}$ above ground level (AGL) with a horizontal speed of approximately $4 \mathrm{~m} / \mathrm{s}$. Images were collected continuously at an interval of $2.4 \mathrm{~s}$, which resulted in an $88 \%$ forward and $75 \%$ sideways overlap of the collected images with a GSD of approximately $8 \mathrm{~cm}$. The images were aligned and ortho- and georectified in PhotoScan Professional 1.2.6 (AgiSoft LLC, St. Petersburg, Russia). After georeferencing, each image at each spectral band was separately exported as an orthorectified GeoTIFF file with a 5 
meter ground pixel size, capturing a representative proportion of potato rows in each pixel.

Then, using the position of the UAV at the time of image acquisition and the position of the georeferenced ground pixels, the observation angles of each ground pixel were calculated according to the equations given in [148]. Since georeferencing requires pixels to be captured in at least two images, this thus resulted in a multiangular dataset for each pixel. Due to the movement of the UAV during the acquisition of an image and the used FPI technology (section 5.2.2), each pixel was observed from a slightly different position in each spectral band.

Table 5.2. Centre wavelengths and Full-Width-at-Half-Maximum (FWHM) of the 16 spectral bands that were sampled in the study of [148] and used in this study.

\begin{tabular}{ccc}
\hline Band & Centre Wavelength [nm] & FWHM [nm] \\
\hline 1 & 500.2 & 14.8 \\
2 & 547.0 & 13.2 \\
3 & 558.8 & 13.0 \\
4 & 568.8 & 12.9 \\
5 & 657.6 & 13.0 \\
6 & 673.6 & 13.2 \\
7 & 705.8 & 13.1 \\
8 & 739.0 & 19.4 \\
9 & 782.8 & 18.5 \\
10 & 791.6 & 18.4 \\
11 & 810.3 & 18.1 \\
12 & 829.0 & 17.8 \\
13 & 847.8 & 17.6 \\
14 & 864.7 & 17.4 \\
15 & 878.7 & 17.3 \\
16 & 894.7 & 17.1 \\
\hline
\end{tabular}

To demonstrate the angular coverage of this method, figure 5.2 shows the observation geometry for the center pixel in each experimental plot at band 6 $(673.6 \mathrm{~nm})$. On average, the center pixels of the experimental plots were captured from 28 different positions per spectral band, covering the hemisphere up to around $30^{\circ}$ from zenith. A pixel being captured in 28 camera positions implies that the pixel was viewed from 448 (number of camera positions times number of bands) different viewing angles. As can be seen in the polar plots of figure 5.2, the nadir position was never exactly captured. In this paper, we compare multi-angular inversions with inversions based on nadir data. For the nadir inversion, we used the pixels that were captured the closest to the nadir position. For the readability of this paper, we refer to such position closest to nadir as the nadir position. The reader is referred to [148] for a more detailed description of the processing of the data and the calculation of the viewing angles. 


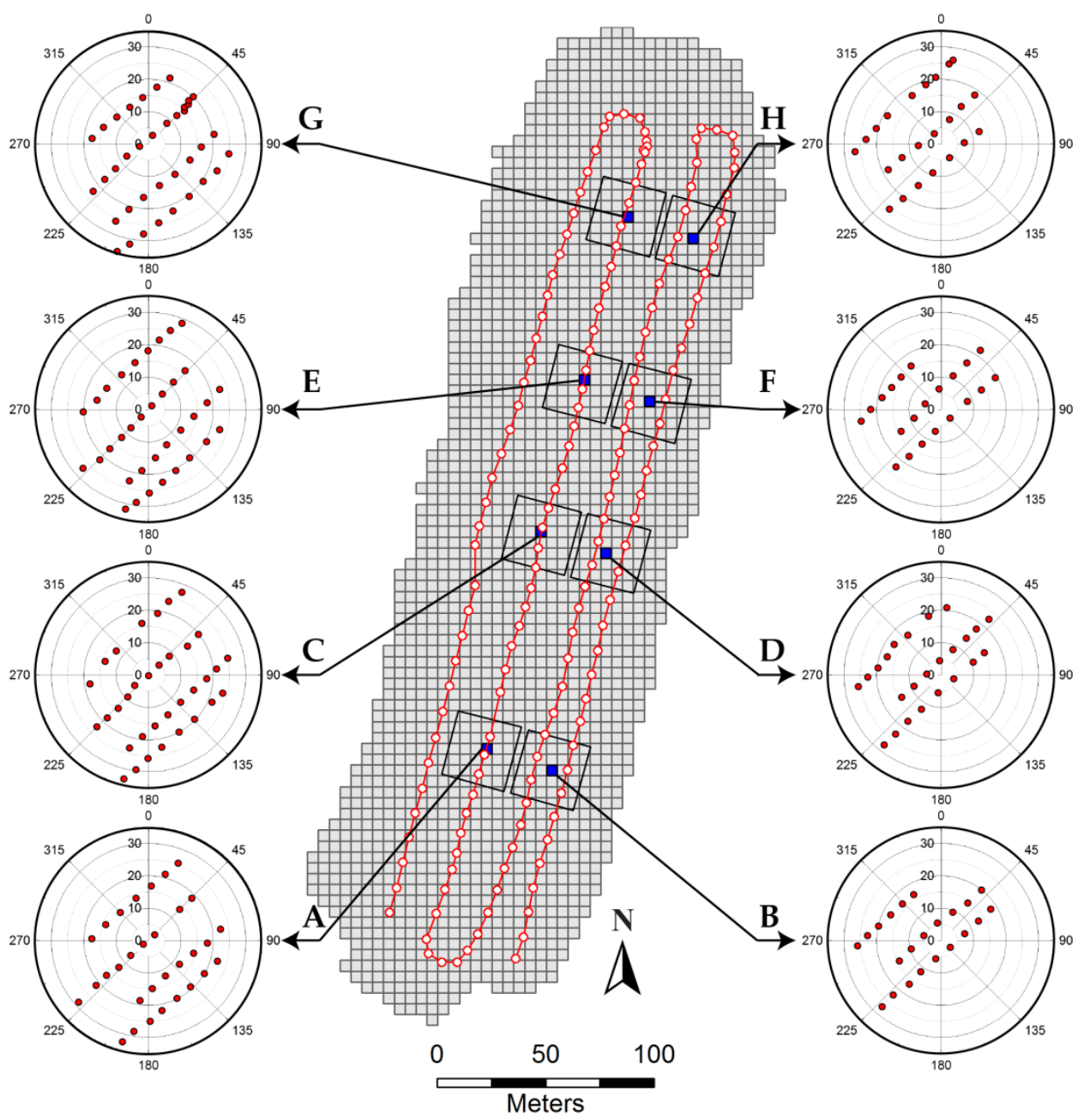

Figure 5.2. Angular coverage of the center pixels (blue pixels) in the experimental plots $\mathrm{A}-\mathrm{H}$. The red circles in the polar plots indicate the viewing angles from which the center pixels were observed. The azimuth angles of the polar plots are relative azimuth angles, where $0^{\circ}$ is the forward scattering direction and $180^{\circ}$ is the backward scattering direction. The red line indicates the path that the UAV flew over the study area. The open circles on this line indicate the positions at which the camera collected an image. The grey raster-background indicates the mapped area during the UAV flight with $5 \times 5$ meter pixels.

\subsubsection{Goniometer viewing angles}

To represent an ideal case where a wide range of well distributed viewing angles are available, we simulated data according to several goniometer angular sampling configurations. Sandmeier [147] suggested that an angular configuration with viewing angles at $30^{\circ}$ azimuth and $15^{\circ}$ zenith resolution covering the hemisphere 
would capture the reflectance anisotropy of a target surface in detail. This angular sampling resolution was therefore the hypothetical baseline of the angular configurations that we tested. However, since most variation in anisotropy is typically observed in the principal plane, we also tested the effect of a denser sampling resolution (every $5^{\circ}$ zenith) in the principal plane. To create a realistic angular sampling scheme, we did not do any simulations at the hotspot position, because in practice it is difficult to take measurements at this position, since the sensor will typically be between the illumination source and the target surface. However, we did test the effect of additional observations around the hotspot position, according to the angular sampling as in [77]. To study the influence of the range of VZAs that are covered, we tested all angular configurations up to a VZA of respectively $30^{\circ}, 60^{\circ}$ or $75^{\circ}$, which are VZAs that can be covered by most goniometer systems $[79,20,15,150,27]$. Four different angular configurations were tested for these three VZA ranges resulting in 12 different angular sampling schemes (table 5.3 and figure 5.3). The angular configurations can be divided into: 1) viewing angles only in the principal plane at $5^{\circ}$ zenith angle interval [configurations $1,5,9$ ], 2) viewing angles covering all azimuth directions at a $30^{\circ}$ angular resolution in $15^{\circ}$ zenith steps [configurations 2,6 , and 10], 3) the same configuration of viewing angles as angular configuration 2 , with a higher density (every $5^{\circ}$ ) of viewing angles in the principal plane [configurations 3,7 , and 11], and 4) the same angular configuration as angular configuration 3, with 3 additional viewing angles around the hotspot position [configurations 4, 8, and 12].

Table 5.3. The different angular configurations used to create the simulated goniometer data. $\mathrm{PP}=$ principal plane, $\mathrm{Hs}=$ hotspot.

\begin{tabular}{|c|c|c|c|c|}
\hline $\begin{array}{c}\text { Angular } \\
\text { configuration }\end{array}$ & $\begin{array}{l}\text { Azimuth } \\
\text { range } \\
\text { (step size) }\end{array}$ & $\begin{array}{l}\text { Zenith range } \\
\text { (step size) }\end{array}$ & $\begin{array}{c}\text { Additional viewing } \\
\text { angles }\end{array}$ & $\begin{array}{l}\text { Number } \\
\text { of angles }\end{array}$ \\
\hline 1 & $0^{\circ}-180^{\circ}\left(180^{\circ}\right)$ & $0^{\circ}-30^{\circ}\left(5^{\circ}\right)$ & - & 12 \\
\hline 2 & $0^{\circ}-180^{\circ}\left(30^{\circ}\right)$ & $0^{\circ}-30^{\circ}\left(15^{\circ}\right)$ & - & 14 \\
\hline 3 & $0^{\circ}-180^{\circ}\left(30^{\circ}\right)$ & $0^{\circ}-30^{\circ}\left(15^{\circ}\right)$ & $5^{\circ}$ steps zenith in PP & 21 \\
\hline 4 & $0^{\circ}-180^{\circ}\left(30^{\circ}\right)$ & $0^{\circ}-30^{\circ}\left(15^{\circ}\right)$ & $\begin{array}{c}5^{\circ} \text { steps zenith in PP } \\
\text { and } 3 \text { positions } \\
\text { around the Hs }\end{array}$ & 25 \\
\hline 5 & $0^{\circ}-180^{\circ}\left(180^{\circ}\right)$ & $0^{\circ}-60^{\circ}\left(5^{\circ}\right)$ & - & 22 \\
\hline 6 & $0^{\circ}-180^{\circ}\left(30^{\circ}\right)$ & $0^{\circ}-60^{\circ}\left(15^{\circ}\right)$ & - & 28 \\
\hline 7 & $0^{\circ}-180^{\circ}\left(30^{\circ}\right)$ & $0^{\circ}-60^{\circ}\left(15^{\circ}\right)$ & $5^{\circ}$ steps zenith in PP & 42 \\
\hline 8 & $0^{\circ}-180^{\circ}\left(30^{\circ}\right)$ & $0^{\circ}-60^{\circ}\left(15^{\circ}\right)$ & $\begin{array}{c}5^{\circ} \text { steps zenith in PP } \\
\text { and } 3 \text { positions } \\
\text { around the Hs }\end{array}$ & 45 \\
\hline 9 & $0^{\circ}-180^{\circ}\left(180^{\circ}\right)$ & $0^{\circ}-75^{\circ}\left(5^{\circ}\right)$ & - & 28 \\
\hline 10 & $0^{\circ}-180^{\circ}\left(30^{\circ}\right)$ & $0^{\circ}-75^{\circ}\left(15^{\circ}\right)$ & - & 35 \\
\hline 11 & $0^{\circ}-180^{\circ}\left(30^{\circ}\right)$ & $0^{\circ}-75^{\circ}\left(15^{\circ}\right)$ & $5^{\circ}$ steps zenith in PP & 53 \\
\hline 12 & $0^{\circ}-180^{\circ}\left(30^{\circ}\right)$ & $0^{\circ}-75^{\circ}\left(15^{\circ}\right)$ & $\begin{array}{c}5^{\circ} \text { steps zenith in PP } \\
\text { and } 3 \text { positions } \\
\text { around the Hs }\end{array}$ & 56 \\
\hline
\end{tabular}




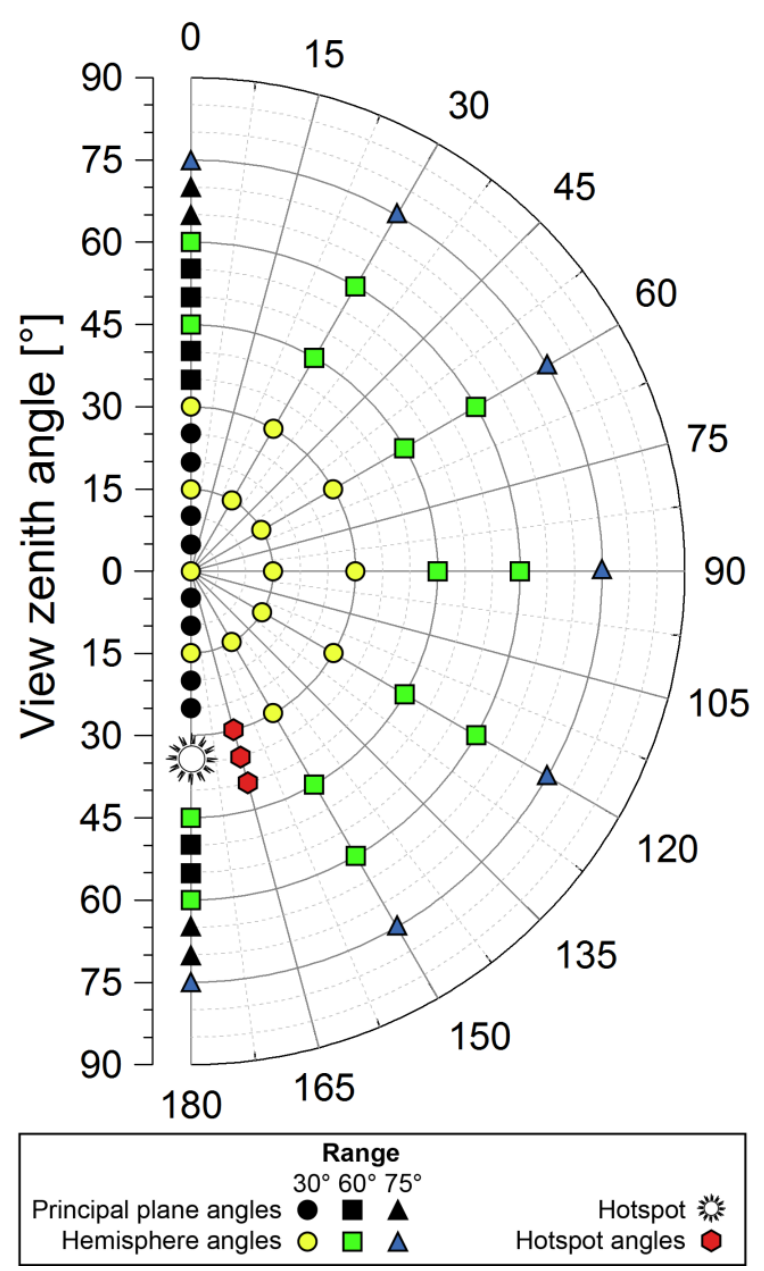

Figure 5.3. Graphical representation of the 12 multi-angular sampling configurations as in table $5.3 .0^{\circ}$ is the forward scattering direction and $180^{\circ}$ is the backward scattering direction.

\subsubsection{Simulation of spectral data using PROSAIL}

To evaluate whether multi-angular observations can lead to improved estimations of LAI and LCC, we simulated multi-angular reflectance datasets with the PROSAIL model for both the UAV viewing angles (section 5.2.3) and goniometer angular configurations (section 5.2.4) using the hsdar-package in $R$ [151]. We used this simulated multi-angular data to invert the PROSAIL model and estimate LAI and LCC, and compared the estimated LAI and LCC values with the field measurements that were used to simulate the data (section 5.2.6). Improvement of the LAI and LCC estimations was assessed by comparing the estimations of LAI and LCC based on solely nadir data and based on multi-angular data. 
Table 5.4. Parameter settings for the simulation of the UAV and goniometer datasets.

\begin{tabular}{|c|c|c|c|c|}
\hline \multicolumn{5}{|c|}{ UAV and goniometer data simulation } \\
\hline Parameter & Description & units & Value / range & steps \\
\hline $\mathrm{N}$ & Leaf structure parameter & - & 2 & - \\
\hline Cbrown & $\begin{array}{c}\text { Leaf brown pigment } \\
\text { content }\end{array}$ & - & 0 & - \\
\hline $\mathrm{C}_{\mathrm{w}}$ & $\begin{array}{l}\text { Leaf equivalent water } \\
\text { thickness }\end{array}$ & $\mathrm{cm}$ & 0.01 & - \\
\hline $\mathrm{C}_{\mathrm{m}}$ & Leaf dry matter content & $\mu \mathrm{g} / \mathrm{cm}^{2}$ & 0.009 & - \\
\hline psoil & Dry/Wet soil factor & - & 0 & - \\
\hline rsoil & $\begin{array}{l}\text { Background soil } \\
\text { reflectance }\end{array}$ & - & $\begin{array}{l}\text { Measurement } \\
\text { of field sample }\end{array}$ & - \\
\hline ALA & Average leaf angle & $\circ$ & 60 & - \\
\hline hspot & Hotspot parameter & - & 0.33 & - \\
\hline tts & Solar zenith angle & $\circ$ & 34 & - \\
\hline \multicolumn{5}{|c|}{ UAV data simulation } \\
\hline LAI & Leaf Area Index & $\mathrm{m}^{2} / \mathrm{m}^{2}$ & As in table 5.1 & As in table 5.1 \\
\hline LCC & Leaf chlorophyll content & $\mu \mathrm{g} / \mathrm{cm}^{2}$ & As in table 5.1 & As in table 5.1 \\
\hline tto & Observer zenith angle & $\circ$ & As in figure 5.2 & As in figure 5.2 \\
\hline psi & Relative azimuth angle & $\circ$ & As in figure 5.2 & As in figure 5.2 \\
\hline \multicolumn{5}{|c|}{ Goniometer data simulation } \\
\hline LAI & Leaf Area Index & $\mathrm{m}^{2} / \mathrm{m}^{2}$ & $1-8$ & 0.5 \\
\hline LCC & Leaf chlorophyll content & $\mu \mathrm{g} / \mathrm{cm}^{2}$ & $20-80$ & 1.0 \\
\hline tto & Observer zenith angle & $\circ$ & As in table 5.3 & As in table 5.3 \\
\hline psi & Relative azimuth angle & $\circ$ & As in table 5.3 & As in table 5.3 \\
\hline
\end{tabular}

To perform the PROSAIL simulations for the UAV viewing angles, we used the LAI and LCC values of the experimental plots as given in table 5.1. The average leaf angle (ALA) was set to $60^{\circ}$, which was found to be the best performing angle for the measured data after testing a wide range of ALAs. Moreover, an ALA of $60^{\circ}$ is in line with research done by Casa and Jones [152], who found ALAs of $57^{\circ}$ to $65^{\circ}$ for several potato canopies. The leaf brown pigment content parameter $\left(\mathrm{C}_{\text {brown }}\right)$ was set at 0 , since there were no brown leaves observed in the potato canopy after visual inspection. To model the soil background signal, a soil sample was collected in the field and its spectrum was measured with an ASD FieldSpec 3 spectrometer (Analytical Spectral Devices, Boulder, CO). The solar zenith angle (tts) was set to $34^{\circ}$, similar to the sun angle during the UAV measurements. To perform the simulations for goniometer data, the LAI parameter was varied between 1 and 8 $\mathrm{m}^{2} / \mathrm{m}^{2}$ in steps of $0.5 \mathrm{~m}^{2} / \mathrm{m}^{2}$ and the LCC was varied between 20 and $80 \mu \mathrm{g} / \mathrm{cm}^{2}$ in steps of $1.0 \mu \mathrm{g} / \mathrm{cm}^{2}$, resulting in 915 combinations of LAI and LCC values. An overview of the parameter settings is given in table 5.4 .

After the simulations with the PROSAIL model at the viewing angles and parameter settings of table 5.4, we spectrally resampled the simulated spectra to match the spectral characteristics of the Rikola camera (table 5.2). Then, Gaussian noise was added to the simulated spectra to mimic inaccuracies of the measurements. Since there is no standard amount of noise documented in literature that is typically added to the spectra [153-156], and because the objective of our study was not to 
investigate the influence of the amount of noise added, we only applied Gaussian noise with a mean of 0 and a standard deviation of 0.05 .

\subsubsection{Model inversion}

The datasets produced with PROSAIL were inverted by means of a general-purpose, iterative optimizing routine in $R$ that allows box-constraints using the optim function. The function was set to minimize the root-mean-squared-error (RMSE) between the measured and modelled reflectance factors over all available viewing angles and all available wavelengths (equation 5.1).

$$
R M S E=\sqrt{\sum_{i=1}^{N_{v a}} \sum_{j=1}^{N_{w l}} \frac{\left(R_{\text {meas }}-R_{\text {modelled }}\right)^{2}}{N}}
$$

Where $N_{v a}$ is the number of viewing angles, $N_{w l}$ is the number of wavelengths, $R_{\text {meas }}$ is the measured reflectance factor, $R_{\text {modelled }}$ is the modelled reflectance factor and $N$ is the total number of observations (number of wavelengths times the number of bands). In the case of the simulated UAV and goniometer data, $R_{\text {meas }}$ refers to the simulated reflectance factors based on the parameter settings as in table 5.4. In the case of the measured data, $R_{\text {meas }}$ refers to the reflectance factors measured by the Rikola camera.

During the inversion, the LAI and LCC parameters were estimated while all other parameters were kept at fixed values as presented in table 5.4. The LAI and LCC parameter ranges were restricted between $0-10 \mathrm{~m}^{2} / \mathrm{m}^{2}$ and $0-120 \mu \mathrm{g} / \mathrm{cm}^{2}$, respectively. We specifically chose to set the parameter ranges beyond the measured and simulated range, to allow the fitting algorithm to look for solutions outside of the boundaries of data simulation. The initial value for the LAI parameter was set at $5 \mathrm{~m}^{2} / \mathrm{m}^{2}$ and the initial value for the LCC parameter was set at $40 \mu \mathrm{g} / \mathrm{cm}^{2}$.

\subsection{Results}

\subsubsection{Measured UAV data}

Figure 5.4a and 5.4b show the estimated LAI values from PROSAIL inversion based on nadir UAV data and multi-angular UAV data, respectively. The differences in fertilization zones (figure 5.2) clearly show up in the maps. Especially plot C, the plot that did not receive any initial and additional fertilization, stands out with its low LAI value. Also plot $\mathrm{G}$ and $\mathrm{H}$, the plots that received a relatively low amount of additional fertilization, have a clearly lower LAI. Moreover, the tractor-tracks, which ran through the center of the plots, have a lower LAI than the potato canopy as well. This is especially apparent in the fertilization zone of plot $\mathrm{E}$ and $\mathrm{F}$.

For LCC, the general patterns were the other way around: Plot $\mathrm{C}$ had a clearly higher LCC than the other plots, which had similar LCC values (figure 5.4d,e). In addition, 
the LCC in the tractor-tracks was also estimated to be higher than in the potato canopy.

In general, the inversion based on nadir data and based on multi-angular data yielded very similar results. A difference map (the multi-angle map subtracted from the nadir map, figure 5.4c) shows that the differences between the nadir LAI and multi-angular inverted LAI are in general small (average difference of $-0.05 \pm 0.43$ $\mathrm{m}^{2} / \mathrm{m}^{2}$ ). The estimation of LCC based on nadir (figure $5.4 \mathrm{~d}$ ) and based on multiangular data (figure 5.4e) also resulted in small differences (figure 5.4f), with an average difference of $-0.29 \pm 3.0 \mu \mathrm{g} / \mathrm{cm}^{2}$.
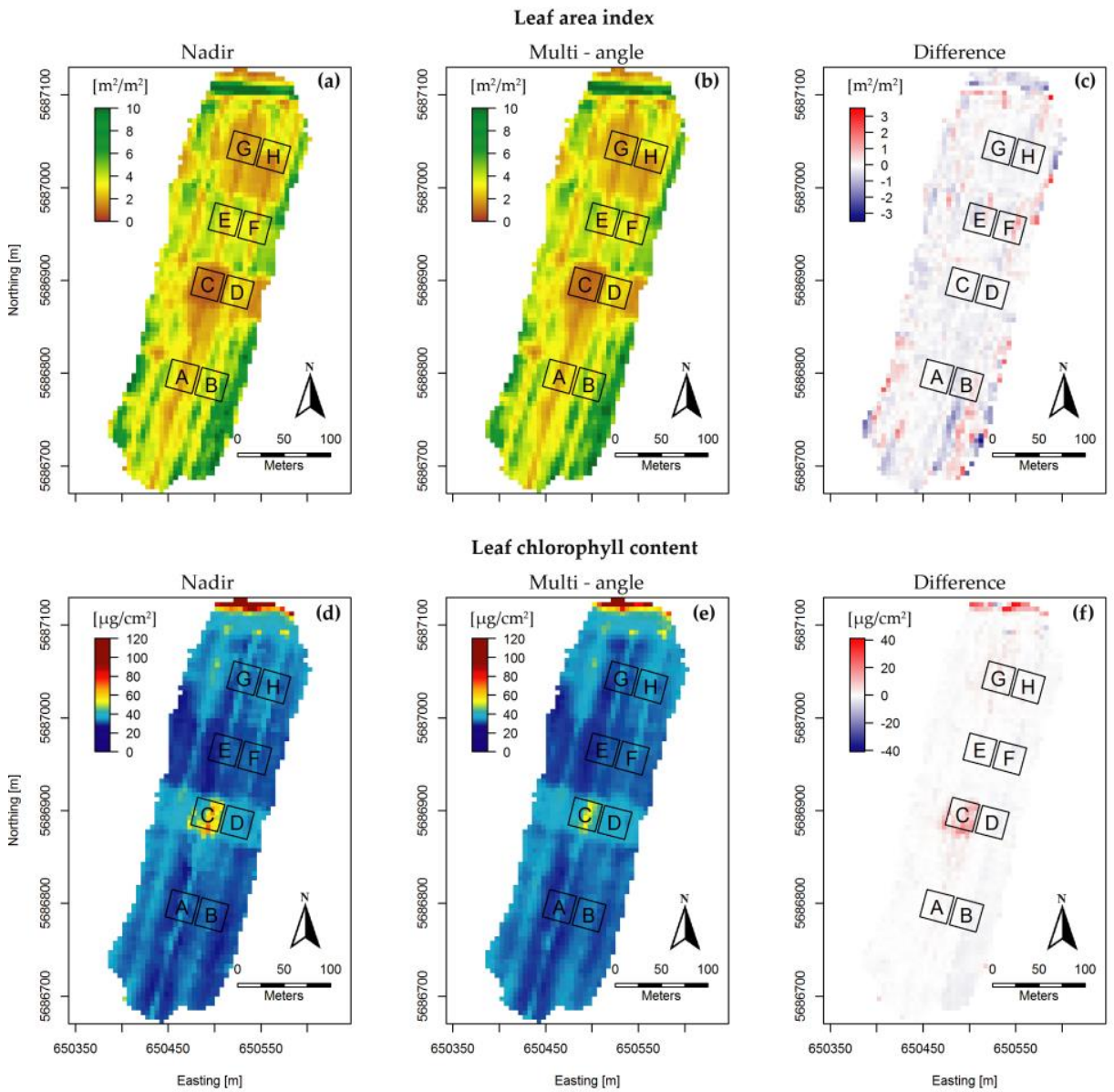

Figure 5.4. LAI estimated from nadir data (a) and from multi-angular data (b). LCC estimated from nadir data (d) and from multi-angular data (e). Panel (c) and (f) show the difference between the estimations based on nadir data and based on multiangular data for LAI and LCC, respectively. The maps are in WGS84 UTM 31N projection. 
Using the data collected in the field as a reference, we studied the accuracy of the inversion based on the nadir and based on the multi-angular UAV data. We calculated the average estimated LAI and LCC value of all pixels within each experimental plot to obtain an average estimated parameter value per plot. The pixels that contained tractor tracks were excluded from the analysis. Results are shown in figure 5.5, comparing the estimated LAI and LCC from model inversion with the field measurements for both nadir and multi-angular observations for the eight different plots yielding the RMSE with respect to the 1:1 line. Since deviations from the 1:1 line could be large but systematic, also a regression line was calculated yielding an $\mathrm{R}^{2}$ value using this regression line.

When only the reflectance spectrum measured at nadir position was used for the inversion, LAI was retrieved with an RMSE of $0.7 \mathrm{~m}^{2} / \mathrm{m}^{2}$ and for the regression line an $\mathrm{R}^{2}$ of 0.91 was obtained. LCC was retrieved with an RMSE of $17.35 \mu \mathrm{g} / \mathrm{cm}^{2}$ and an $\mathrm{R}^{2}$ of 0.09 (figure 5.5 top panels). When the multi-angular data was used for the inversion, there was a small decrease of the RMSE for the LAI retrieval to 0.65 $\mathrm{m}^{2} / \mathrm{m}^{2}$ (figure 5.5 bottom panels). The correlation between the measured and estimated LAI remained the same. For the LCC retrieval, a small decrease of the RMSE and an increase of the $\mathrm{R}^{2}$ was observed.
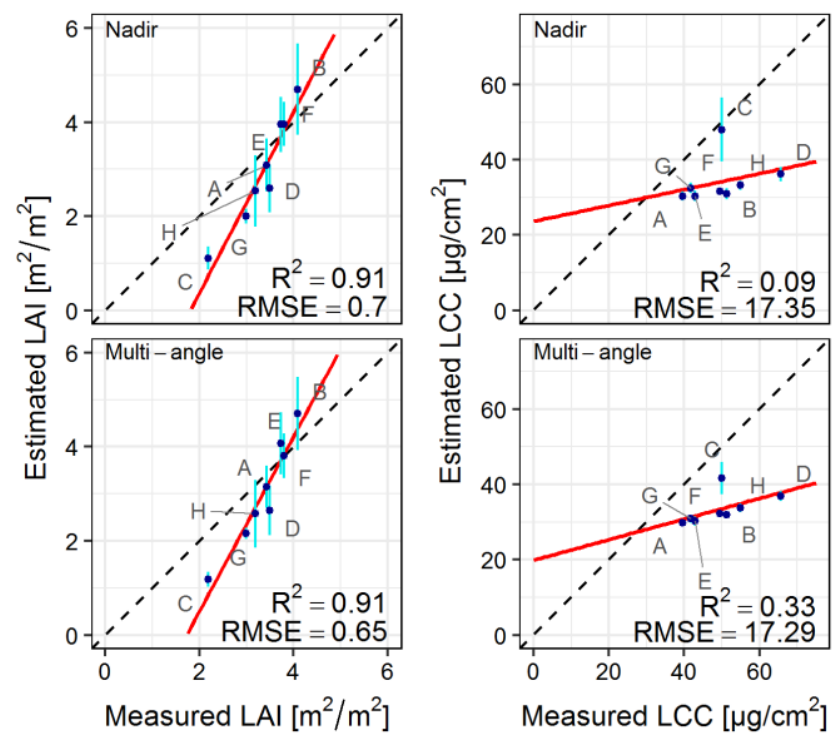

Figure 5.5. Scatter plots showing the measured and estimated LAI (left panels) and LCC (right panels), based on inversion using solely the nadir spectrum (top panels) and the multi-angular spectra (bottom panels). The letters $\mathrm{A}-\mathrm{H}$ indicate the experimental plots (figure 5.1). The red line indicates the regression line and the vertical cyan-colored lines indicate the standard deviation of the estimated LAI and LCC values of the pixels within each plot. Note that for the LCC estimates the standard deviations for most experimental plots were very small and therefore not always visible. 
These results show that the improvement of using multi-angular measurements over using only nadir measurements was small, although the standard deviations of the individual retrieved LAI and LCC measurements were lower for the multiangular measurements than for the nadir measurements. The retrieved LAI estimates (figure 5.5, left panels) show that low LAI values are underestimated by the PROSAIL inversion, whereas high values are overestimated. The $\mathrm{R}^{2}$ for the calculated regression line is high, showing the good potential of LAI retrieval. It must be stated that conclusions should be drawn with care since these results are based on just eight different plots. Results for LCC retrieval are worse, because the range of LCC values was limited. The retrieved LCC value for plot $C$ was larger than for all other plots (also compare figure 5.4), which might be caused by ambiguity occurring when retrieving LAI and LCC simultaneously. Plot C also had the lowest LAI retrieved by model inversion, and it was most underestimated. Such underestimation might be compensated by an overestimation of LCC.

\subsubsection{Simulated UAV data}

To assess the case where a realistic range and distribution of viewing angles are available and where we are sure that the used RTM can reproduce the input data, we repeated a similar exercise as in section 5.3.1 based on multi-angular reflectance data simulated with the PROSAIL model according to the viewing angles in figure 5.2. For each experimental plot, we estimated LAI and LCC by inverting the PROSAIL data based on the reflectance spectrum simulated at nadir position and compared this with the estimation of LAI and LCC based on the multi-angular spectra. When the inversion was performed solely based on the nadir spectrum, LAI was retrieved with an RMSE of $0.64 \mathrm{~m}^{2} / \mathrm{m}^{2}$ and an $\mathrm{R}^{2}$ of 0.66 for the fitted regression line. LCC was retrieved with an RMSE of $24.37 \mu \mathrm{g} / \mathrm{cm}^{2}$ with an $\mathrm{R}^{2}$ of 0.28 , respectively (figure 5.6 , top panels). This indicates, that even though the input data was simulated by the PROSAIL model- and we can thus exclude that there was a disagreement between the input data and the model used for inversion- only using a nadir spectrum does not produce very accurate estimates of LAI and LCC.

When the inversion was performed using data simulated at the viewing angles according to figure 5.2, the estimations of LAI and LCC strongly improved. LAI was retrieved with an RMSE of $0.08 \mathrm{~m}^{2} / \mathrm{m}^{2}$. The fitted regression line almost matched the $1: 1$ line and the $\mathrm{R}^{2}$ was 0.98 . LCC was retrieved with and RMSE of $2.43 \mu \mathrm{g} / \mathrm{cm}^{2}$ and an $\mathrm{R}^{2}$ of 0.96 . Also for LCC the fitted regression line was close to the 1:1 line. This indicates that when multi-angular data are used for inversion and it is certain that the model can reproduce the input data, an improved accuracy of LAI and LCC retrievals can be obtained by using multi-angular data. 

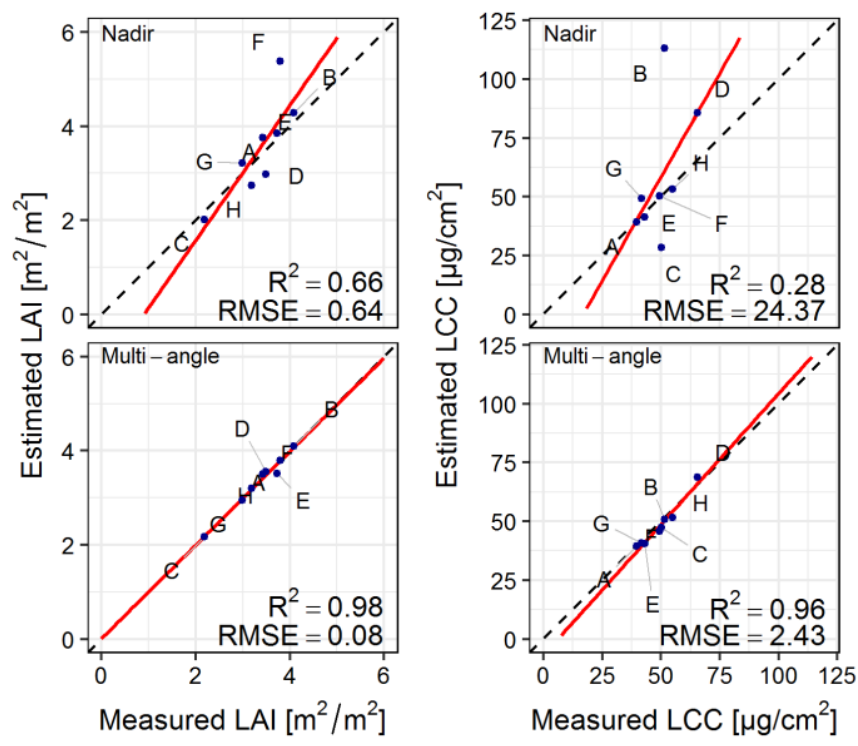

Figure 5.6. Scatter plots showing the measured and estimated LAI (left panels) and LCC (right panels), based on inversion using solely the spectrum simulated at nadir position (top panels) and the spectra simulated at the multiple viewing angles as in figure 5.2 (bottom panels). The letters $\mathrm{A}-\mathrm{H}$ indicate the experimental plots (figure 5.1).

\subsubsection{Simulated goniometer data}

Retrieval of the 915 different combinations of LAI and LCC was performed by inversion of the PROSAIL model solely based on the simulated nadir spectrum and the one based on the spectra simulated at the 12 multi-angular configurations according to table 5.3. Inversion results were already strongly improved in terms of RMSE and $\mathrm{R}^{2}$ when additional viewing angles were used for VZA up to $30^{\circ}$ (figure 5.7). All regression lines were close to the $1: 1$ line, meaning larger $R^{2}$ values will coincide with smaller RMSE values. When only the nadir spectrum was used for inversion, an $\mathrm{R}^{2}$ of 0.79 and RMSE of $1.11 \mathrm{~m}^{2} / \mathrm{m}^{2}$ was obtained for the estimation of LAI, and an $\mathrm{R}^{2}$ of 0.40 and RMSE of $22.67 \mu \mathrm{g} / \mathrm{cm}^{2}$ for LCC. When viewing angles in the principal plane were used for the inversion, the $\mathrm{R}^{2}$ increased to 0.97 and 0.89 for LAI and LCC, respectively. The RMSE decreased to $0.36 \mathrm{~m}^{2} / \mathrm{m}^{2}$ and $6.39 \mu \mathrm{g} / \mathrm{cm}^{2}$ for LAI and LCC, respectively. Using viewing angles covering the hemisphere up to a VZA of $30^{\circ}$ performed slightly better than when only viewing angles in the principal plane were used, indicated by the RMSE of $0.35 \mathrm{~m}^{2} / \mathrm{m}^{2}$ and $6.27 \mu \mathrm{g} / \mathrm{cm}^{2}$ for LAI and LCC, respectively. The combination of viewing angles in the principal plane and the viewing angles covering the hemisphere performed slightly better than the viewing angles in the principal plane and the viewing angles covering the hemisphere separately. Finally, another small improvement was observed when the three observation angles close to the hotspot were used in addition to the viewing angles in the principal plane and the viewing angles covering the 
hemisphere, yielding an RMSE of $0.25 \mathrm{~m}^{2} / \mathrm{m}^{2}$ and $4.51 \mu \mathrm{g} / \mathrm{cm}^{2}$ for the estimation of LAI and LCC, respectively.

An overall pattern that could be observed was that when the number of viewing angles used for the inversion increased, estimations of LAI and LCC improved. Especially in the range $1-5 \mathrm{~m}^{2} / \mathrm{m}^{2}$, the estimated LAI parameter values were very close to the initial values when more viewing angles were used for inversion (figure 5.7, left panels). At higher LAI values, the deviation from the 1:1 line increased. A similar trend was observed for the estimation of LCC. For this parameter, especially estimations in the $20-50 \mu \mathrm{g} / \mathrm{cm}^{2}$ range were very close to the initial LCC values (figure 5.7, right panels).

When only the nadir spectrum was used for the inversion, there were some inversions where the fitting algorithm saturated and the maximum LAI or LCC value was found as best solution. With additional viewing angles used for the inversion, this saturation did almost not occur anymore (compare the top panels of figure 5.7 with the other panels). 

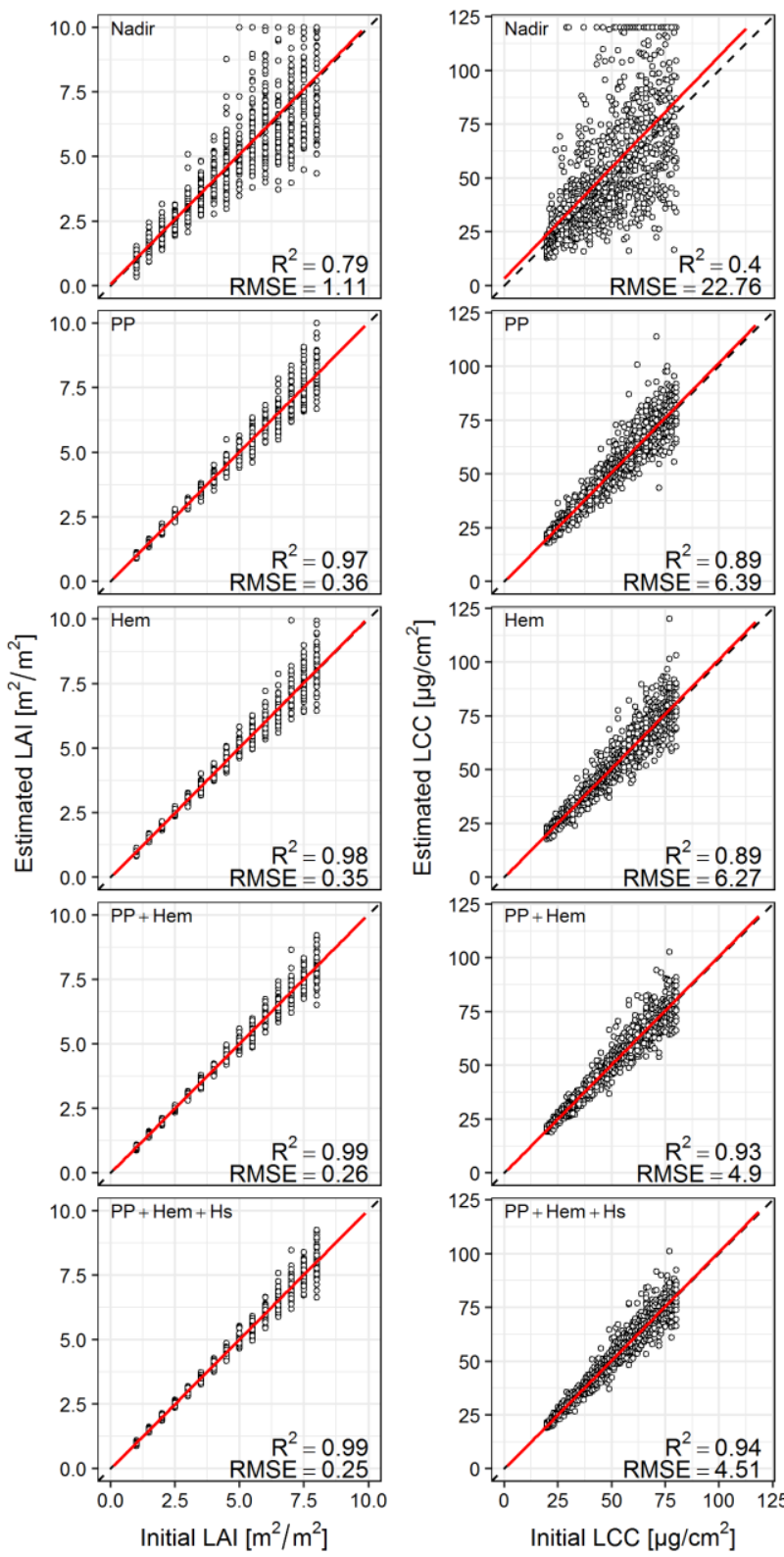

Figure 5.7. Scatter plots showing the initial and estimated LAI (left panels) and LCC (right panels), based on inversion using solely the nadir spectrum (top panels) and the spectra at different angular configurations up to a VZA of $30^{\circ}$ (lower panels). $\mathrm{N}=$ 915. The dashed lines indicated the 1:1-line and the red line indicates the regression line. $\mathrm{PP}=$ principal plane, $\mathrm{Hem}=$ hemisphere, and $\mathrm{Hs}=$ hotspot.

Similar trends were observed when the angular configurations with VZAs up to $60^{\circ}$ and $75^{\circ}$ were used for the inversion. In general, the best estimations of LAI and LCC 
were obtained when VZAs up to $75^{\circ}$ were introduced (figure 5.8). When VZAs up to $75^{\circ}$ were used for the inversion, combined with a higher density of viewing angles in the principal plane and around the hotspot, a reduction of the RMSE of $84.05 \%$ and $88.26 \%$ was obtained as compared to the use of only the nadir spectrum for LAI and LCC, respectively.

(a)

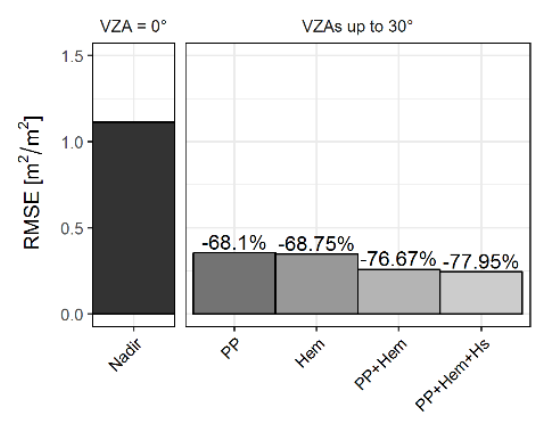

(b)

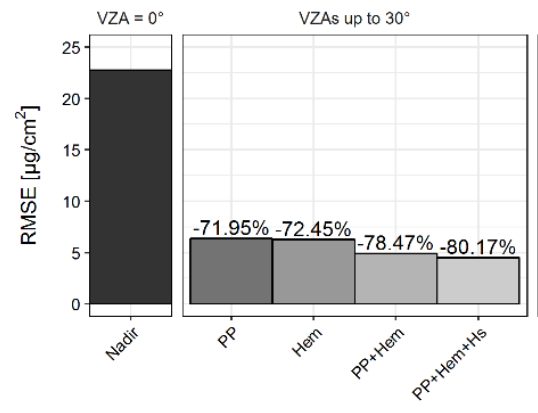

Leaf Area Index
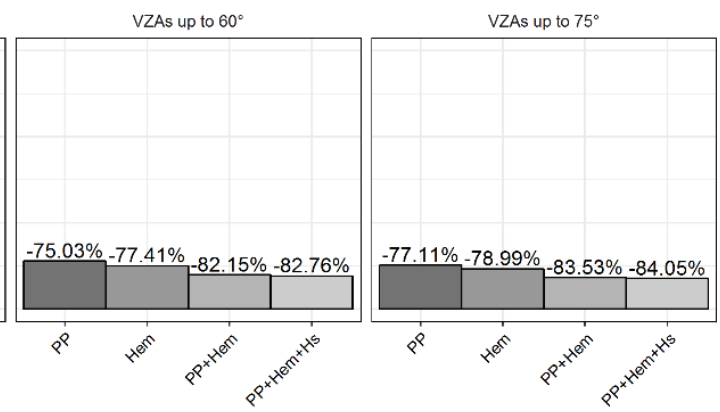

Leaf chlorophyll content
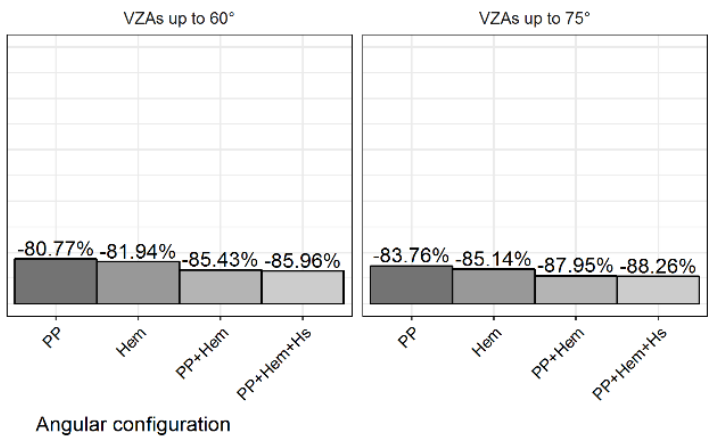

Figure 5.8. RMSE between the initial and inverted LAI (a) and LCC (b) values for the spectra simulated at the different angular configurations. The percentages on top of the bars indicate the decrease of the RMSE, compared to inversion solely based on the spectrum simulated at nadir position. $\mathrm{PP}=$ principal plane, $\mathrm{Hem}=$ hemisphere, and Hs = hotspot.

\subsection{Discussion}

In this paper, we studied whether using multi-angular reflectance data compared to solely nadir data could improve estimations of LAI and LCC by inversion of the PROSAIL model. We tested this for a measured dataset collected by a camera mounted on a UAV and for several simulated datasets, where a UAV angular sampling configuration and several goniometer angular configurations were used as basis for the simulations. Overall, inversion of PROSAIL yielded good results for LAI retrieval, but rather poor results for LCC retrieval. Low LAI values were underestimated by the model inversion, whereas high LAI values were 
overestimated. The range in LCC values was limited, making LCC retrieval cumbersome. Notably, the experimental plot with the lowest LAI retrieved (plot C) showed the largest retrieved LCC value. RTMs like PROSAIL contain sets of input variables that always appear in combination, such as products between variables as is, e.g., the case with LAI and LCC [157]. Similar reflectance spectra simulated by PROSAIL may therefore correspond to a wide range of solutions for LAI and LCC. A compensation between variables may occur for LAI and LCC estimates. An underestimate of LAI often goes together with an overestimation of LCC, and vice versa.

For the measured dataset, which was collected over a potato field, small improvements of the estimations of LAI and LCC were obtained when inversion based on the reflectance spectra measured at all available viewing angles was compared to inversion based on the spectrum collected at nadir position. There are several possible explanations for having only small improvements in the case of the measured UAV data. For instance, there is the possibility that PROSAIL was not able to adequately reproduce spectral and/or anisotropy characteristics of the measured potato canopy. Although the PROSAIL model has been successfully used before to estimate biophysical and biochemical parameters of potato canopies $[158,88]$, the model has difficulties reproducing the strong anisotropy caused by shadow effects in for example row crops [141]. However, since the potato canopy for most of the experimental plots was fully developed and was completely covering the soil at the time of data collection, these row induced shadow effects in our data were minimal. In addition, the selection of parameter settings could not have represented the actual situation properly. However, we have tested several other combinations of fixed and free parameter settings (results not shown), but the combination of the LAI and LCC as free parameters, together with the fixed values for the other parameters as in table 5.4, provided the best results. Care should be taken with drawing conclusions on these results, because only 8 plots were included in this part of the study.

For the simulated datasets, on the contrary, large improvements were obtained when multi-angular reflectance spectra were used for inversion of the PROSAIL model to estimate LAI and LCC. The simulated UAV dataset provided a realistic angular sampling configuration that can easily be obtained by frame-based cameras. Since the input data in this case was created by the PROSAIL model, it can be excluded that the model was not able to reproduce the input data. Inversion of this dataset based on multi-angular data led to strong improvements in the estimations of LAI and LCC as compared to nadir data. Moreover, the simulated data for the goniometer angular configurations demonstrated that when more viewing angles, more well distributed viewing angles, and viewing angles up to greater zenith angles were available for inversion, the estimations of LAI and LCC improved even further.

In this study, we did not search for the optimal number and positions of viewing angles that are needed to obtain the best inversion results. Weiss et al. [144] showed that a limited number of sampling directions resulted in improvement of vegetation parameter estimations. Using more observation directions would add 
more redundant information, and thereby more noise to the input data, which in the end resulted in more uncertainty in the parameter retrieval. A similar effect was also observed in a study of Dorigo [141], who studied the improvement of LAI and LCC estimation of a cotton crop based on multi-angular CHRIS data. In this study, up to five viewing angles were iteratively added to be used for the inversion of the PROSAIL model. Adding the first four viewing angles resulted in a decrease in RMSE between measured and estimated LAI and LCC. However, when the fifth observation angle was used, a strong increase in RMSE was observed. Dorigo [141] attributed this decrease in accuracy to the inability of the PROSAIL model to correctly reproduce the anisotropy at this angle and argued that the model probably compensated for this with an unnatural variation of the LAI and LCC parameters. It could be possible that the PROSAIL model was not capable of correctly reproducing the reflectance anisotropy of the potato canopy that we used in this study, which therefore might have resulted in the limited improvement of the estimations of the LAI and LCC parameters based on multi-angular data.

The processing time for iterative inversion techniques are in general quite long, especially when the number of input data points or the number of variables to be estimated is large. As an example, the estimation of LAI and LCC based on inversion of a nadir spectrum ( 1 viewing angle times 16 bands, thus 16 input data points) took on average $1 \mathrm{~s}$, while inversion based on multi-angular spectra measured by the UAV (as example 28 observation angles; thus $28 \times 16$ bands $=448$ input data points) took on average $27 \mathrm{~s}$. Moreover, iterative optimization techniques can suffer from finding a local minimum instead of a global minimum as solution to the inverse problem. However, we applied an iterative optimization technique in this study, since doing so made it possible to directly use all the available viewing angles. Other inversion techniques, such as lookup tables (LUTs) which do not suffer from finding a local minimum as solution, or artificial neural networks (ANNs) require to be remade or retrained, respectively, every time a new viewing angle is introduced. This issue could be overcome when for example a model-to-model approach [159] is applied. In such an approach, a more simple BRDF model that uses just a couple of parameters to describe the surface reflectance anisotropy is fitted through the multi-angular measurements and its parameters are estimated. Subsequently, the obtained parameters are used to simulate multi-angular reflectance data at a fixed set of viewing directions, which then can be used to invert the RTM. Such a strategy makes it possible to test different inversion techniques on the multi-angular data. However, it has to be noted that a model-to-model approach might also introduce new sources of error or uncertainty caused by the additional modelling step.

Although the improvements of the estimations of LAI and LCC using the UAV data were small compared to the improvements obtained with the simulated data, it is shown in this study that the easy to obtain multi-angular views that are captured in the overlap of frame-based cameras can be used for improved estimation of LAI and LCC. In future studies, an exercise as performed in this study should be undertaken for different types of vegetation or crops to confirm this. Moreover, it should be tested whether the estimation of other vegetation parameters benefit from multiangular observations as well. 


\subsection{Conclusions}

In this paper, we studied the additional value of multi-angular observations for the retrieval of LAI and LCC by inversion of the PROSAIL model for three cases: 1) for data captured during a UAV flight, 2) for data simulated at the angular configurations that were captured during this UAV flight, and 3) for data simulated at angular configurations that are typically captured by goniometer systems. The results for the simulated data showed a clear improvement of the retrieved parameters in terms of RMSE and $\mathrm{R}^{2}$ when multi-angular observations were used, as compared to solely nadir data. The generally observed trend was that when more viewing angles, more well-distributed viewing angles, and viewing angles up to larger zenith angles were available, the most accurate inversion results were obtained.

Inversion of the PROSAIL model based on measured multi-angular reflectance data of a potato field, collected by a frame-camera mounted on a UAV, also showed improvements of the estimations of LAI and LCC, compared to when solely nadir data was used. Although the improvements were not as large as for the simulated data, it does indicate that the multi-angular views, that can easily be collected by frame-cameras on UAVs, can help to improve parameter estimation, making such an approach interesting for operational use. In this paper, we only estimated parameters of a potato crops. Future studies should focus on the improvement of parameter retrieval by the inversion based on multi-angular reflectance data for different vegetation targets as well.

Acknowledgements: This research was funded by a grant from the User Support Programme Space Research (GO/12-15) in The Netherlands. The authors would like to thank the Wageningen University \& Research Unmanned Aerial Remote Sensing Facility (UARSF) for providing the UAV and sensor system used in this study. In addition, the authors thank Jacob van den Borne for the preparation of and access to the experimental field and Marnix van den Brande for doing the LAI and LCC measurements. 



\title{
Chapter 6
}

\author{
Synthesis
}




\subsection{Main results}

The main objective of this thesis was to study the information content of the reflectance anisotropy signal for improved crop parameter retrieval and to study how UAVs can be used for this. Based on this objective, four research questions were defined (section 1.3). In this chapter, each research question will be addressed based on the main findings as presented in chapters 2-5.

\subsubsection{What is the effect of soil moisture content on reflectance anisotropy?}

This research question was addressed in chapter 2. Soil moisture content (SMC) is an important parameter for many environmental, ecological, and agricultural practices, and therefore knowledge on the distribution of soil moisture at high temporal and spatial scales is of interest for many applications. Optical remote sensing methods to study SMC typically relate changes in reflectance to soil moisture based on empirically or physically based models. However, in most studies soil reflectance anisotropy is not taken into account. Moreover, little is known about the information content of the reflectance anisotropy signal in relation to SMC.

To study the effects of SMC, we developed a laboratory experiment, where multiangular reflectance measurements in the 350-2500 $\mathrm{nm}$ wavelength region were taken of several soil samples at a variety of SMC levels, using a robotic goniometer setup [15]. At the start of the experiment, the soil samples were saturated with water and they were placed on a digital balance in the centre of the goniometer setup. Multi-angular reflectance measurements of the soil samples were repetitively taken at a set of 60 positions covering the hemisphere up to view zenith angles of $65^{\circ}$, while the soil samples were left to dry out. Using the known weight of the dry soil samples and of the added water, and the weight at the time that a reflectance measurement was taken, the SMC level was linked to each reflectance measurement. The measured anisotropy patterns at each wavelength in the captured wavelength range was parameterized and quantified by the parameters of the Rahman-Pinty-Verstraete (RPV) model [66]. Then, the relation between the RPV parameters and SMC was studied to see if, and how, the SMC level affects reflectance anisotropy.

It is known that when the SMC level increases, the reflectance decreases $[45,47]$. This has been confirmed in several studies, e.g. [42,54,53], and was also clearly visible in our measurements. The higher the SMC level, the lower the observed reflectance factor. The amplitude parameter $\rho_{0}$ of the RPV model, which closely relates to the nadir reflectance, followed this trend. At higher SMC levels, a lower value of $\rho_{0}$ was found, especially in the water absorption bands around 1450 and $1900 \mathrm{~nm}$. This resulted in a maximum $\mathrm{R}^{2}$ of 0.622 at $1921 \mathrm{~nm}$ between SMC and the $\rho_{0}$ parameter.

The relation between the parameters of the RPV model that describe the reflectance anisotropy, namely the asymmetry parameter $(\Theta)$, which controls the intensity of 
forward and backward scattering, the Minnaert parameter $(k)$, which controls the bell-shape and bowl-shape of the anisotropy curve, and the hotspot parameter $\left(\rho_{c}\right)$, which approximates the reflectance around the hotspot position, were also studied. Especially, the $\Theta$ parameter showed a strong relationship to SMC level. Where dry soil samples showed a higher reflectance in the backward scattering direction, with a maximum reflectance at the hotspot position, soil samples with a higher SMC level showed less pronounced backward scattering. This relationship was observed for the whole measured wavelength range, but was most pronounced at the NIR and SWIR region, resulting in a maximum observed $\mathrm{R}^{2}$ of 0.797 between SMC and the $\Theta$ parameter at $2123 \mathrm{~nm}$. Besides that the results of the performed experiment demonstrated that SMC has a strong influence on reflectance anisotropy and therefore needs to be taken into account when optical remote sensing methods are used to study SMC, the results also indicated that reflectance anisotropy contains information on SMC and might therefore be used for improved estimation of SMC using optical remote sensing methods.

\subsubsection{How can UAVs be used to study reflectance anisotropy?}

This research question was addressed in chapters 3 and 4 . Whereas goniometers are suitable devices for multi-angular measurements of flat surfaces, like soils, such as measured in chapter 2, their ground-based design typically limits the sensortarget distance, which does not allow them to take representative multi-angular measurements of surfaces with a strong three dimensional structure, such as vegetation or crops. To overcome this issue, we studied the opportunities to capture multi-angular measurements using a UAV. The flexibility of UAVs enables measurements taken with a greater distance between the target and sensor, allowing sampling the anisotropy of surfaces with a strong three dimensional structure. Until now, the number of attempts to perform multi-angular measurements using UAVs is very limited.

We explored the collection of UAV-based multi-angular reflectance measurements by testing two different sensor systems. The first system, which was used in chapter 3, was the Hyperspectral Mapping System (HYMSY) [91]. This system consist of a pushbroom spectrometer, a photogrammetric camera, and a miniature GPS-Inertial Navigation System. The spectrometer has a field of view (FOV) of $42.4^{\circ}$, making it possible to capture reflectance at off-nadir view angles.

To capture the anisotropy of several crops (barley, potato, and winter wheat), we developed a flight plan where the UAV was programmed to fly to the centres of the crop fields. In the centre of the fields, the UAV was programmed to hover at approximately 10 meters above ground level (AGL), while rotating around its vertical axis. In this way, the spectrometer, which was mounted tilted at a $12^{\circ}$ angle under the UAV, was able to capture measurements up to a view zenith angle (VZA) of approximately $30^{\circ}$, while covering all azimuth directions. Using this measurement strategy we assumed that the area that was captured in the FOV of the sensor was homogeneous. This strategy is similar as, for example, applied at goniometers like AMSPEC [28-30] and PARABOLA [31]. By fitting the RPV model 
through the measurements, we were able to parameterize and quantify the anisotropy of the studied crops in the measured wavelength range covered by the sensor (450 - $915 \mathrm{~nm}$ at a 5-nm interval), enabling to study anisotropy at a hyperspectral resolution.

Although the above described measurement strategy allows capturing the average anisotropy signal of the area covered by the sensor, it does not allow for an insight in the spatial distribution or variation of the reflectance anisotropy within a field, i.e., it does not allow for the creation of anisotropy maps. In order to do this, we tested a second system in chapter 4 , where a snapshot camera was used to collect multi-angular observations. The used camera applies Fabry-Perot interferometer (FPI) technology to collect snapshot imagery at programmatically selectable bands. The camera has a horizontal and vertical FOV of $36.5^{\circ}$ and collects images of 1010 $\times 1010$ pixels. The measurement strategy applied in this chapter was to extract the reflectance factors at view angles that were captured in the overlapping parts of collected images during a standard mapping flight.

By applying this measurement strategy over a potato field, we were able to capture views in the study area up to approximately $30^{\circ}$ zenith angles, covering all azimuth directions. Pixels in the centre of our study area were captured in up to 40 different images, which thus resulted in up to 40 different view angles for these pixels. By fitting the RPV model through the multi-angular measurements, we were able to quantify the anisotropy of each pixel in our study area. Moreover, this enabled us to create anisotropy parameter maps, which gave an insight in the spatial distribution of anisotropy patterns within the area covered by the UAV.

In chapter 3 and 4, we have shown for two different sensor systems that reflectance anisotropy can be captured with a UAV. This showed that UAV-based multi-angular measurements could be an interesting alternative for traditional goniometer or satellite-based multi-angular measurements.

\subsubsection{What is the effect of canopy development on reflectance anisotropy?}

This research question was addressed in chapters 3 and 4 . To explore the information content of reflectance anisotropy of agricultural crops, it is important to study what the effects of canopy development of crops are on reflectance anisotropy. To do so, we applied the two UAV-based multi-angular measurement strategies as described in section 6.1.2 on multiple days during the growing season.

Multi-angular measurements with the pushbroom system (chapter 3) were performed twice during the growing season of 2015 over a barley, potato, and winter wheat canopy. There was a time difference of 22 days between the measurements during which several canopy developments had taken place for the different crops. Whereas on the first measurement day, the top layer of both the barley and the winter wheat canopy was mainly formed by leaves, on the second measurement day both crops had developed grain heads. The grain heads of the barley crops had large awns and were bended downwards, while the grain heads of 
the winter wheat did not have such large awns and were vertically orientated. The potato canopy had developed from an open row structure, where the soil was still visible between the potato rows, to a canopy that was completely covering the soil.

On the first measurement day, before the development of grain heads, both barley and winter wheat showed very similar anisotropic reflectance patterns, which were mainly dominated by backward scattering. This backward scattering was strongest in the visible wavelengths, with emphasis on the red wavelength region, where shadow effects are strongest due to high absorption of light by chlorophyll in this region. This backward scattering was less pronounced in the NIR region as a result of the relatively low absorption of light by chlorophyll, and therefore higher transmittance and reflectance by the leaves in this wavelength region, and thus less pronounced shadows. When plotting the $\Theta$ parameter as a function of wavelength, this resulted in an anisotropy signature having the shape of a vegetation spectral signature. On the second measurement day, when the grain heads had developed, a change in anisotropy was observed too. This was especially the case for the barley canopy, of which the top layer was now formed by downward bended grain heads with large awns, where a strong decrease of backward scattering intensity was observed in the visible wavelength region.

For the potato canopy also clear changes in anisotropy were observed when the canopy had developed from an open row structure on the first measuring day to a closed canopy on the second measuring day. On the first day, strong shadows caused by the row structure of the canopy were observed, resulting in a negative $\Theta$ parameter value over the whole sampled wavelength range. On the second day, the space between the potato rows had disappeared due to the growth of the potato plants, which resulted in a strong decrease of anisotropy being most obvious through a decrease of backward scattering as indicated by a $\Theta$ value of approximately 0 (indicating no dominating forward or backward scattering reflectance) over the whole measured wavelength region.

Multi-angular measurements performed with a snapshot camera mounted on a UAV were presented in chapter 4 . The measurements were taken on two days during the growing season of 2016 over a potato field where different initial (before planting) and sensor-based (during crop growth) fertilization regimes were applied to eight experimental plots, which resulted in differences in the growth of the potato plants and thus in different stages of canopy development within each experimental plot. By fitting the RPV model through the pixels that were captured in multiple images, we were able to create anisotropy parameter maps of the potato field. The relation between leaf area index (LAI) and canopy cover, and the obtained RPV parameters in each plot, was determined to study their influence reflectance anisotropy. On the first measurement day, when the potato rows were still being formed (i.e., the potato plants were still quite small and did not form a complete row, especially in experimental plots where no or low fertilization was applied), we observed the strongest backward scattering intensity in the experimental plots that had the highest canopy cover. In the plots with higher cover, there were no gaps between the individual plants due to the bigger size of the potato plants. Plots with a lower canopy cover, where there were still gaps in the potato rows, showed less 
pronounced backward scattering. The gaps in the potato rows resulted in less strong row-induced shadow effects, and therefore a less pronounced contrast between the forward and backward scattering views, and thus a less pronounced backward scattering intensity.

On the second measurement day, 40 days later, we observed in general a decrease of backward scattering with an increase of canopy cover. In this case, this was the result of the closing of the space between consecutive potato rows, which had as a result that the strong shadows caused by the potato rows were no longer present. This effect was similar as was observed in the measurements of chapter 3.

Overall, the UAV-based multi-angular measurements presented in chapter 3 and 4 demonstrated for three different crops that canopy development results in changes in anisotropy. Therefore, it indicates that the reflectance anisotropy signal contains information on canopy development.

\subsubsection{How can reflectance anisotropy be used to improve crop parameter retrieval?}

This research question was addressed in chapter 5 . Using the RPV model in chapter 3 and 4, we demonstrated that different crops and different growth stages of these crops result in a variety anisotropy patterns. This indicates that reflectance anisotropy contains information on crop type and their growing stage. In chapter 5 , we studied if using multi-angular observations can be used to improve crop parameter retrieval using the PROSAIL model.

We compared numerical inversions of the PROSAIL model to estimate LAI and leaf chlorophyll content (LCC) based on nadir data and based on multi-angular data. We tested this for the dataset of chapter 4 and for several datasets simulated by the PROSAIL model at a variety of view angle configurations that can be captured with a snapshot camera mounted on a UAV, and that can be captured using typical goniometer systems. For the measured dataset, we observed minor improvements between the estimated LAI and LCC when multi-angular data were used for the inversion of the PROSAIL model as compared to when nadir data were used. Reasons for the minor improvements can be, for example, limitations of the PROSAIL model to accurately reproduce the measured spectral and angular reflectance [141], or errors and uncertainties spectral measurements -either way, a possible mismatch between the measurements and the model. For the simulated datasets, we observed very large improvements in the estimation of LAI and LCC when multi-angular data were used for the inversion as compared to when only nadir data were used. When multi-angular data simulated at view angles that can easily be captured during a typical UAV flight, as is demonstrated in chapter 4, were used for inversion, already large improvements of the estimated LAI and LCC were achieved. Moreover, when more view angles, more widely distributed view angles, and view angles up to greater zenith angles were available, the inversion accuracy increased even further. This indicated that when it can be assumed that the used model can reproduce the input data (as was the case with these data because they 
were made by the PROSAIL model) improvements in parameter retrieval can be achieved.

Although improvements of LAI and LCC were not as pronounced for the measured dataset as for the simulated dataset, we did show that crop parameter retrieval can be improved when multi-angular data are used. Moreover, the multi-angular data used in this chapter were collected during a standard UAV mapping flight, meaning that it is relatively easy to obtain these multi-angular views, without the need to fly complicated flight patterns or changing the view direction of the sensor during the flight. UAV-based studies that focus on parameter retrieval might therefore benefit from the multiple view angles that are 'always' captured by frame cameras.

\subsection{Reflection and Outlook}

\subsubsection{Reflection}

The research performed in this thesis was motivated by the need for high quality estimation of physical vegetation parameters at a fine spatial resolution for applications such as agriculture. Moreover, this thesis explored the use of UAVs to capture reflectance anisotropy, as an alternative for traditional ground-based goniometer and space-borne multi-angular measurements. The results presented in this thesis contribute to the following topics:

1. Insights in the effects of surface characteristics on reflectance anisotropy, as demonstrated for SMC content in chapter 2 and for several vegetation parameters as demonstrated in chapter 3 and 4 .

2. Innovative measurement strategies to perform multi-angular measurements using a UAV with different sensor systems, as demonstrated in chapter 3 and 4.

3. Additional value of multi-angular observations for improved parameter retrieval as demonstrated in chapter 5 .

\subsubsection{Soil moisture content}

In chapter 2, the effects of SMC on reflectance anisotropy have been studied. In the experiment performed for this chapter, the soil moisture content was the only varied parameter, which made it possible to isolate the contribution of SMC to reflectance anisotropy. Soil reflectance anisotropy, however, is the result of several other soil properties, such as soil surface roughness, and soil organic matter content [41]. Especially, the confounding effects of soil surface roughness and SMC make it difficult to quantify the contributions of each parameter individually when both are varied.

Due to the flat surface of the soil samples studied in this thesis, the soil samples could be measured using a ground-based goniometer. To study the combined contributions of both SMC and soil roughness on reflectance anisotropy, a measurement approach with a frame-based camera mounted on a UAV, such as 
demonstrated in chapter 4, could be applied. The overlap of the images collected by a frame-based camera does not only allow for the creation of a multi-angular spatial dataset, it would also allow for the creation a detailed digital surface model (DSM), which can provide a reliable representation of soil surface roughness $[160,161]$. The multi-angular data combined with the DSM can provide an insight in the reflectance anisotropy and surface roughness on a pixel basis, and would, in combination with a suitable sampling scheme for in situ measurements of SMC, be a feasible method to study the combined effect of SMC and soil roughness on reflectance anisotropy.

\subsubsection{Measurement scale}

In the pre-launch phase of the Multi-angle Imaging Spectrometer (MISR) and the Compact High Resolution Imaging Spectrometer (CHRIS) and the period after the launch, reflectance anisotropy and BRDF received a lot of attention. Especially the additional information content of multi-angular observations with respect to vegetation structure was widely studied in this period $[13,162,32]$. The period after this, with the emergence of techniques such as LiDAR, which can provide accurate information of the structure of vegetation [163], the interest in this topic faded away again. However, recently, a new trend in the field of remote sensing has emerged with the advent of UAVs. UAVs have rapidly developed into a robust and widely used tool for remote sensing research. UAVs provide, in contrast to satellites, data at a very high spatial resolution, making them in particular useful for applications such as precision agriculture.

The photogrammetric processing of data collected by UAVs relies on the overlap of images that are collected during a UAV flight. This overlap results in multi-angular views of pixels that are captured in the multiple images, due to the movement of the UAV during data collection. In most studies, these multiple view angles are ignored. However, in this thesis, we have shown that there is information on surface parameters in these multi-angular views. Depending on the used sensor and the height flown AGL during data collection, multi-angular measurements with a very high spatial resolution, up to a centimetre scale, can be obtained. For example, figure 6.1, shows multi-angular data collected with a UAV over four reference panels using a similar strategy as applied in chapter 4. A flight with different headings over the panels was executed to collect observations that cover multiple azimuth angles (figure $6.1 \mathrm{~b}$ ). The panels were $50 \times 50 \mathrm{~cm}$ and were captured at a ground sampling distance (GSD) of approximately $8 \mathrm{~cm}$. Panels 1 and 3 were covered with gravel, and panels 2 and 4 were covered with glossy and matte paint, respectively (figure 6.1c,d). The $k, \Theta$, and $\rho_{c}$ parameters obtained by fitting the RPV parameters through the multi-angular data in the red band $(658 \mathrm{~nm})$ clearly showed distinctive values for the different panels (figure 6.1e). Of the four panels, the gravel covered panels showed the strongest backward scattering characteristics, indicated by the strongest negative $\Theta$ values for the pixels of these panels (figure 6.1e, middle image). Interestingly, the smooth wooden edges of the panels displayed forward scattering characteristics, as can be seen by the positive $\Theta$ values at the edges of the panels. The matte-painted panel, like the gravel covered 
panels, also showed backward scattering characteristics, however, less pronounced as indicated by the slightly negative $\Theta$ values (i.e. $\Theta$ values just below 0 ) for this panel. The glossy panel, on the contrary, showed strong forward scattering characteristics, indicated by the positive $\Theta$ values for the pixels of this panel. Moreover, the bowl / bell shape parameter $(k)$, and the hotspot parameter $\left(\rho_{c}\right)$ also indicated distinctive anisotropy patterns for the different panels.

(a)

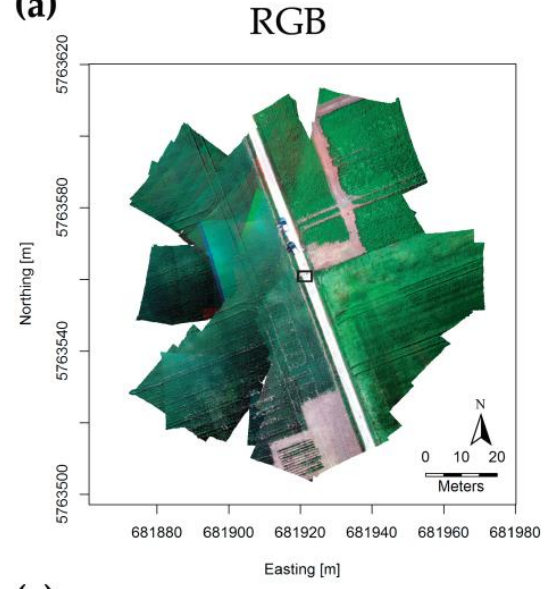

(c)
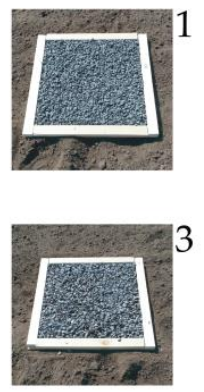

(e)
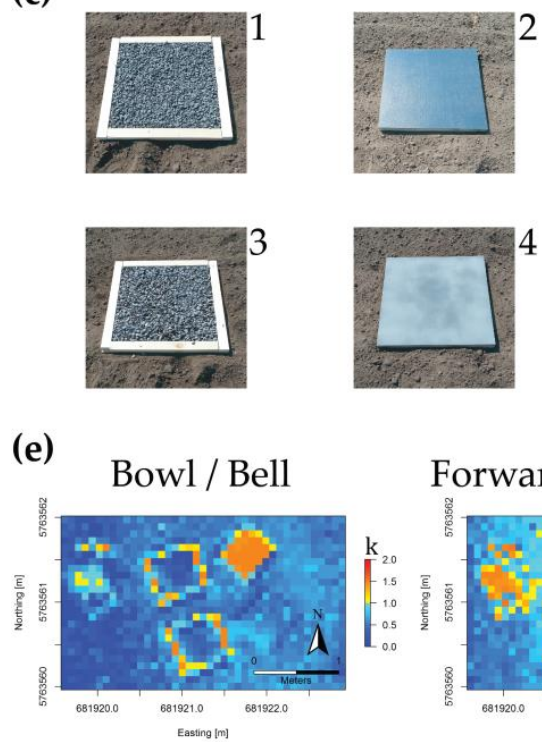

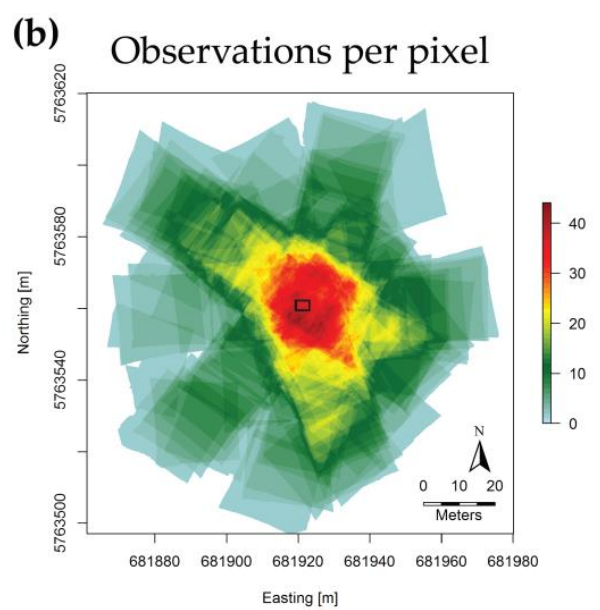

(d)

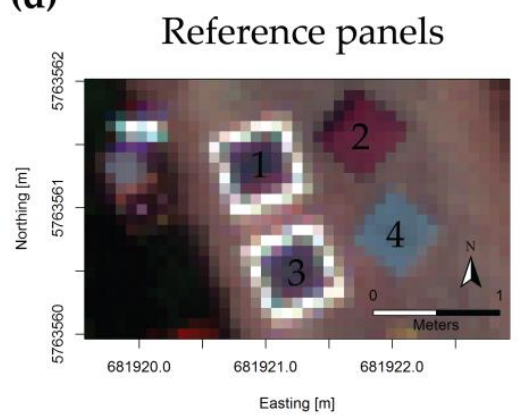

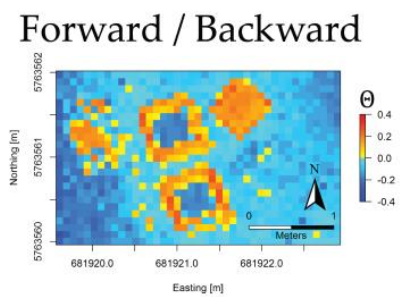

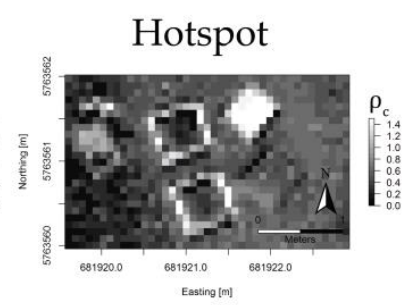

Figure 6.1. A stack of individual ortho- and georectified RGB composite images collected during a UAV flight over four reference panels (a) and the number of observations for each pixel covered during this flight (b). The black rectangle indicates the location of the reference panels. Photos of the reference panels (c) and a close-up RGB composite of the panels at a GSD of approximately $8 \mathrm{~cm}$ (d). The $\Theta, k$, and $\rho_{c}$ parameters obtained by fitting the RPV model though the multi-angular views captured by the UAV in the red band $(658 \mathrm{~nm})$, similar as done in chapter 4 (e). 
The fine GSD that can be obtained by sensors carried by UAVs provides a whole new spatial scale for multi-angular measurements as opposed to satellite-based multiangular measurements, which typically have a coarse spatial resolution, and goniometers that usually only provide information of the reflectance anisotropy of a single point. The spatial support of UAV-based measurements with frame-based cameras allows for the sampling of reflectance anisotropy at the plant level, making it therefore possible to study in detail the effect of vegetation parameters on reflectance anisotropy.

\subsubsection{Zenith angle range}

In this thesis, UAV-based multi-angular measurements have only been collected up to a VZA of approximately $30^{\circ}$. Using multi-angular measurements up to this limited VZA for inversion of the PROSAIL model already improved the estimation of crop parameters, compared to nadir measurements (chapter 5). However, we have also shown using simulated data, that observations at larger VZAs can improve parameter estimation even further. In future studies, sampling up to larger VZAs needs to be explored to capture a more complete view of the reflectance anisotropy and thereby achieve even more improved parameter estimations with measured data. This could, for example, be done by mounting sensors tilted under UAVs, to capture larger VZAs in their FOV, like we already tested with a pushbroom spectrometer in chapter 3 . Such tilting strategy could also be applied to framebased cameras. In this way, pixels near the edge of the FOV of the camera in the direction of the tilt will be viewed at larger zenith angles. Flying with different headings over a target area would thereby result in larger VZAs in multiple azimuth directions. Similarly, mounting a frame-based camera on a gimbal would allow the capturing of larger VZAs if the UAV is flown over and around a target surface while the camera is actively pointed towards the centre of this surface, forming a goniometer angular sampling scheme [36]. Moreover, to get a higher density of angular measurements, different flight patterns that not necessarily focus on mapping, such as demonstrated in figure 6.1, can be explored.

\subsubsection{Anisotropy correction}

Applications such as time series analysis, change detection, and crop growth monitoring require data with high spatio-temporal continuity, consistency, and quality. Currently, a multitude of satellite data is already available and this availability will increase enormously in the near future. To obtain the required temporal resolution for such applications, increased use has to be made of data collected by a combination of sensors onboard different satellites, collected during different orbits and thus at different observation and illumination geometries. This makes information on surface anisotropy of great importance. Multi-sensor data integration techniques currently are requiring algorithms correcting for surface anisotropy effects. An example of such corrected product is the nadir BRDF adjusted reflectance (NBAR) [72,11], which uses multi-angular reflectance data collected by the Moderate Resolution Imaging Spectroradiometer (MODIS) onboard the Terra and Aqua satellites, accumulated over a period of 16 days, to 
determine per pixel the reflectance as if they were observed from nadir view. The multi-angular views that are collected in the overlap of images acquired by a framebased camera on a UV could also be used to determine the reflectance per pixel at a standardized viewing and illumination geometry, which is necessary to make data collected at different time points directly comparable.

In this thesis, it is shown that anisotropy effects not only depend on the type of surface, like crop type or soil, but also on the canopy development stage and soil moisture level, indicating that anisotropy corrections should be highly dynamic. This still requires a lot of research in the future.

\subsubsection{Multi-angular UAV-based measurements for precision agriculture}

The main goal of precision agriculture is to reduce production costs and enhance sustainability while maintaining (or increasing) yields by applying fertilizers, pesticides, or irrigation at the right time and location with the right amounts [164]. Although satellite remote sensing is an important tool for the monitoring of agricultural fields, the relatively low spatial and temporal resolution of satellite products is not sufficient for precision management of crops [165]. The low operational costs and high operational flexibility of UAVs make them an interesting alternative to traditional satellite platforms [166]. Due to their relatively low flight height AGL, UAVs can provide measurements of crops at a centimetre scale, enabling the mapping and monitoring of individual plants.

Currently, most precision agricultural products based on data collected by optical sensors mounted on UAVs are maps of Vegetation Indices (VIs) like the Normalized Difference Vegetation Index (NDVI), representing the crop's health status of a particular field at a particular point in time. VIs are correlated to, for example, nutrient deficiency or water stress, and they can therefore identify areas in a field where plants may not develop up to their full potential. However, VIs do not adequately characterize vegetation as they are sensitive to the used sensor, the time of observation, and the crop under investigation. On the other hand, biophysical parameters adequately reflect the actual state of the crops. For accurate precision management of crops, information on their biophysical characteristics is of great importance to better serve the needs of individual plants.

High resolution UAV-based observations in combination with radiative transfer models (RTMs), such as the PROSAIL model as applied in chapter 5, are a valuable tool for the estimation of biophysical crop parameters. The number of studies where biophysical crop parameters are estimated using RTMs in combination with data collected with UAVs is still very limited and requires more research in the future.

Inversion of RTMs based on spectral data to obtain biophysical parameters is not straight forward [167]. The inversion of RTMs is an ill-posed problem due to measurement and model uncertainties [136], and due to the issue that different combinations of biophysical parameters may result in similar reflectance spectra $[137,138]$. Hence, inversion of an RTM based on spectral data may result in a range 
of possible solutions. As demonstrated in chapter 5, the introduction of multiangular observations can help to improve the estimation of biophysical crop parameters. Frame-based cameras mounted on UAVs collect multi-angular views in the overlapping areas in the images that are captured during data acquisition [148]. These multi-angular observations can be utilized to produce more accurate maps of biophysical crop parameters, which will be beneficial for the field of precision agriculture. Future research should focus on different crops in addition to the potato crop that was studied in chapter 5 , on the estimation of other biophysical parameters that are relevant for precision agriculture in addition to LAI and LCC, and on investigating robust and computationally efficient RTM inversion schemes. 


\section{References}

1. Schaepman, M.E.; Ustin, S.L.; Plaza, A.J.; Painter, T.H.; Verrelst, J.; Liang, S. (2009). Earth system science related imaging spectroscopy-An assessment. Remote Sensing of Environment, 113(SUPPL. 1), S123-S137.

2. Inoue, Y. (2003). Synergy of remote sensing and modeling for estimating ecophysiological processes in plant production. Plant Production Science, 6(1), 3-16.

3. Pinter Jr, P.J.; Hatfield, J.L.; Schepers, J.S.; Barnes, E.M.; Moran, M.S.; Daughtry, C.S.T.; Upchurch, D.R. (2003). Remote sensing for crop management. Photogrammetric Engineering and Remote Sensing, 69(6), 647-664.

4. Schaepman-Strub, G.; Schaepman, M.E.; Painter, T.H.; Dangel, S.; Martonchik, J.V. (2006). Reflectance quantities in optical remote sensingdefinitions and case studies. Remote Sensing of Environment, 103(1), 27-42.

5. Barnsley, M.J.; Allison, D.; Lewis, P. (1997). On the information content of multiple view angle (MVA) images. International Journal of Remote Sensing, 18(9), 1937-1960.

6. Kimes, D.S. (1983). Dynamics of directional reflectance factor distributions for vegetation canopies. Applied Optics, 22(9), 1364-1372.

7. Sandmeier, S.; Müller, C.; Hosgood, B.; Andreoli, G. (1998). Physical mechanisms in hyperspectral BRDF data of grass and watercress. Remote Sensing of Environment, 66(2), 222-233.

8. Schaepman, M.E. (2007). Spectrodirectional remote sensing: From pixels to processes. International Journal of Applied Earth Observation and Geoinformation, 9(2), 204-223.

9. Bacour, C.; Bréon, F.M.; Maignan, F. (2006). Normalization of the directional effects in NOAA-AVHRR reflectance measurements for an improved monitoring of vegetation cycles. Remote Sensing of Environment, 102(3-4), 402-413.

10. Roy, D.P.; Zhang, H.K.; Ju, J.; Gomez-Dans, J.L.; Lewis, P.E.; Schaaf, C.B.; Sun, Q.; Li, J.; Huang, H.; Kovalskyy, V. (2016). A general method to normalize Landsat reflectance data to nadir BRDF adjusted reflectance. Remote Sensing of Environment, 176, 255-271. 
11. Schaaf, C.B.; Gao, F.; Strahler, A.H.; Lucht, W.; Li, X.; Tsang, T.; Strugnell, N.C.; Zhang, X.; Jin, Y.; Muller, J.P.; Lewis, P.; Barnsley, M.; Hobson, P.; Disney, M.; Roberts, G.; Dunderdale, M.; Doll, C.; D'Entremont, R.P.; Hu, B.; Liang, S.; Privette, J.L.; Roy, D. (2002). First operational BRDF, albedo nadir reflectance products from MODIS. Remote Sensing of Environment, 83(1-2), 135-148.

12. Vermote, E.; Justice, C.O.; Bréon, F.M. (2009). Towards a generalized approach for correction of the BRDF effect in MODIS directional reflectances. IEEE Transactions on Geoscience and Remote Sensing, 47(3), 898-908.

13. Asner, G.P. (2000). Contributions of multi-view angle remote sensing to land-surface and biogeochemical research. Remote Sensing Reviews, 18(2), 137-162.

14. Barnsley, M.J.; Lewis, P.; O'Dwyer, S.; Disney, M.I.; Hobson, P.; Cutter, M.; Lobb, D. (2000). On the potential of CHRIS/PROBA for estimating vegetation canopy properties from space. Remote Sensing Reviews, 19(1-4), 171-189.

15. Roosjen, P.P.J.; Clevers, J.G.P.W.; Bartholomeus, H.M.; Schaepman, M.E.; Schaepman-Strub, G.; Jalink, H.; van der Schoor, R.; de Jong, A. (2012). A laboratory goniometer system for measuring reflectance and emittance anisotropy. Sensors, 12(12), 17358-17371.

16. Diner, D.J.; Beckert, J.C.; Reilly, T.H.; Bruegge, C.J.; Conel, J.E.; Kahn, R.A.; Martonchik, J.V.; Ackerman, T.P.; Davies, R.; Gerstl, S.A.W.; Gordon, H.R.; Muller, J.P.; Myneni, R.B.; Sellers, P.J.; Pinty, B.; Verstraete, M.M. (1998). Multi-angle imaging spectroradiometer (MISR) instrument description and experiment overview. IEEE Transactions on Geoscience and Remote Sensing, 36(4), 1072-1087.

17. Barnsley, M.J.; Settle, J.J.; Cutter, M.A.; Lobb, D.R.; Teston, F. (2004). The PROBA/CHRIS mission: A low-cost smallsat for hyperspectral multiangle observations of the earth surface and atmosphere. IEEE Transactions on Geoscience and Remote Sensing, 42(7), 1512-1520.

18. Nag, S. (2013). Design and analysis of distribute NANO-satellite systems for multi-angular, multi-spectral earth observation. In: Proceedings of the International Astronautical Congress, IAC, 2013; pp 4327-4340.

19. Zhang, C.; Kovacs, J.M. (2012). The application of small unmanned aerial systems for precision agriculture: A review. Precision Agriculture, 13(6), 693-712.

20. Biliouris, D.; Verstraeten, W.W.; Dutré, P.; Van Aardt, J.A.N.; Muys, B.; Coppin, P. (2007). A Compact Laboratory Spectro-Goniometer (CLabSpeG) to assess the BRDF of materials. Presentation, calibration and implementation on Fagus sylvatica L. leaves. Sensors, 7(9), 1846-1870. 
21. Feingersh, T.; Ben-Dor, E.; Filin, S. (2010). Correction of reflectance anisotropy: A multi-sensor approach. International Journal of Remote Sensing, 31(1), 49-74.

22. Bachmann, C.M.; Abelev, A.; Montes, M.J.; Philpot, W.; Gray, D.; Doctor, K.Z.; Fusina, R.A.; Mattis, G.; Chen, W.; Noble, S.D.; Coburn, C.; Corl, T.; Slomer, L.; Nichols, C.R.; van Roggen, E.; Hughes, R.J.; Carr, S.; Kharabash, S.; Brady, A.; Vermillion, M. (2016). Flexible field goniometer system: the Goniometer for Outdoor Portable Hyperspectral Earth Reflectance. Journal of Applied Remote Sensing, 10(3), 036012-036012.

23. Coburn, C.A.; Peddle, D.R. (2006). A low-cost field and laboratory goniometer system for estimating hyperspectral bidirectional reflectance. Canadian Journal of Remote Sensing, 32(3), 244-253.

24. Deering, D.W.; Leone, P. (1986). A sphere-scanning radiometer for rapid directional measurements of sky and ground radiance. Remote Sensing of Environment, 19(1), 1-24.

25. Painter, T.H.; Paden, B.; Dozier, J. (2003). Automated spectro-goniometer: A spherical robot for the field measurement of the directional reflectance of snow. Review of Scientific Instruments, 74(12), 5179-5188.

26. Sandmeier, S.R.; Itten, K.I. (1999). A field goniometer system (FIGOS) for acquisition of hyperspectral BRDF data. Geoscience and Remote Sensing, IEEE Transactions on, 37(2), 978-986.

27. Suomalainen, J.; Hakala, T.; Peltoniemi, J.; Puttonen, E. (2009). Polarised multiangular reflectance measurements using the finnish geodetic institute field goniospectrometer. Sensors, 9(5), 3891-3907.

28. Hilker, T.; Coops, N.C.; Nesic, Z.; Wulder, M.A.; Black, A.T. (2007). Instrumentation and approach for unattended year round tower based measurements of spectral reflectance. Computers and Electronics in Agriculture, 56(1), 72-84.

29. Hilker, T.; Nesic, Z.; Coops, N.C.; Lessard, D. (2010). A new, automated, multiangular radiometer instrument for tower-based observations of canopy reflectance (AMSPEC II). Instrumentation Science and Technology, 38(5), 319-340.

30. Tortini, R.; Hilker, T.; Coops, N.C.; Nesic, Z. (2015). Technological advancement in tower-based canopy reflectance monitoring: The AMSPEC-III system. Sensors, 15(12), 32020-32030.

31. Bruegge, C.J.; Helmlinger, M.C.; Conel, J.E.; Gaitley, B.J.; Abdou, W.A. (2000). Parabola III: A sphere-scanning radiometer for field determination of surface anisotropic reflectance functions. Remote Sensing Reviews, 19(1-4), 75-94.

32. Walthall, C.; Roujean, J.L.; Morisette, J. (2000). Field and landscape BRDF optical wavelength measurements: Experience, techniques and the future. Remote Sensing Reviews, 18(2), 503-531. 
33. Sandmeier, S.; Müller, C.; Hosgood, B.; Andreoli, G. (1998). Sensitivity analysis and quality assessment of laboratory BRDF data. Remote Sensing of Environment, 64(2), 176-191.

34. Wang, Z.; Coburn, C.A.; Ren, X.; Teillet, P.M. (2012). Effect of soil surface roughness and scene components on soil surface bidirectional reflectance factor. Canadian Journal of Soil Science, 92(2), 297-313.

35. Colomina, I.; Molina, P. (2014). Unmanned aerial systems for photogrammetry and remote sensing: A review. ISPRS Journal of Photogrammetry and Remote Sensing, 92, 79-97.

36. Burkart, A.; Aasen, H.; Alonso, L.; Menz, G.; Bareth, G.; Rascher, U. (2015). Angular dependency of hyperspectral measurements over wheat characterized by a novel UAV based goniometer. Remote Sensing, 7(1), 725746.

37. Grenzdörffer, G.J.; Niemeyer, F. (2011). UAV based brdf-measurements of agricultural surfaces with pfiffikus. In: International Archives of the Photogrammetry, Remote Sensing and Spatial Information Sciences - ISPRS Archives, 2011; pp 229-234.

38. Hakala, T.; Suomalainen, J.; Peltoniemi, J.I. (2010). Acquisition of bidirectional reflectance factor dataset using a micro unmanned aerial vehicle and a consumer camera. Remote Sensing, 2(3), 819-832.

39. Sandmeier, S.R.; Itten, K.I. (1999). A field goniometer system (FIGOS) for acquisition of hyperspectral BRDF data. IEEE Transactions on Geoscience and Remote Sensing, 37(2 II), 978-986.

40. Wang, L.; Qu, J.J. (2009). Satellite remote sensing applications for surface soil moisture monitoring: A review. Frontiers of Earth Science in China, 3(2), 237-247.

41. Croft, H.; Anderson, K.; Kuhn, N.J. (2014). Evaluating the influence of surface soil moisture and soil surface roughness on optical directional reflectance factors. European Journal of Soil Science, 65(4), 605-612.

42. Lobell, D.B.; Asner, G.P. (2002). Moisture effects on soil reflectance. Soil Science Society of America Journal, 66(3), 722-727.

43. Somers, B.; Gysels, V.; Verstraeten, W.W.; Delalieux, S.; Coppin, P. (2010). Modelling moisture-induced soil reflectance changes in cultivated sandy soils: A case study in citrus orchards. European Journal of Soil Science, 61(6), 1091-1105.

44. Sadeghi, M.; Jones, S.B.; Philpot, W.D. (2015). A linear physically-based model for remote sensing of soil moisture using short wave infrared bands. Remote Sensing of Environment, 164, 66-76. 
45. Angstrom, A. (1925). The albedo of various surfaces of ground. Geogr. Ann., 7, 323-342.

46. Twomey, S.A.; Bohren, C.F.; Mergenthaler, J.L. (1986). Reflectance and albedo differences between wet and dry surfaces. Appl. Opt., 25(3), 431437.

47. Lekner, J.; Dorf, M.C. (1988). Why some things are darker when wet. Applied Optics, 27(7), 1278-1280.

48. Bach, H.; Mauser, W. (1994). Modelling and model verification of the spectral reflectance of soils under varying moisture conditions. In: International Geoscience and Remote Sensing Symposium (IGARSS), 1994; pp 2354-2356.

49. Anderson, K.; Croft, H. (2009). Remote sensing of soil surface properties. Progress in Physical Geography, 33(4), 457-473.

50. Ben-Dor, E.; Chabrillat, S.; Demattê, J.A.M.; Taylor, G.R.; Hill, J.; Whiting, M.L.; Sommer, S. (2009). Using Imaging Spectroscopy to study soil properties. Remote Sensing of Environment, 113(SUPPL. 1), S38-S55.

51. Bartholomeus, H.M.; Schaepman, M.E.; Kooistra, L.; Stevens, A.; Hoogmoed, W.B.; Spaargaren, O.S.P. (2008). Spectral reflectance based indices for soil organic carbon quantification. Geoderma, 145(1-2), 28-36.

52. Muller, E.; Décamps, H. (2001). Modeling soil moisture - Reflectance. Remote Sensing of Environment, 76(2), 173-180.

53. Weidong, L.; Baret, F.; Xingfa, G.; Qingxi, T.; Lanfen, Z.; Bing, Z. (2002). Relating soil surface moisture to reflectance. Remote Sensing of Environment, 81(2-3), 238-246.

54. Nolet, C.; Poortinga, A.; Roosjen, P.P.J.; Bartholomeus, H.M.; Ruessink, G. (2014). Measuring and modeling the effect of surface moisture on the spectral reflectance of coastal beach sand. PLOS ONE, 9(11), e112151.

55. Philpot, W.D. (2010). Spectral reflectance of wetted soils. Symposium: "Art, Science and Applications of Reflectance Spectroscopy (ASARS)".

56. Weidong, L.; Baret, F.; Xingfa, G.; Bing, Z.; Qingxi, T.; Lanfen, Z. (2003). Evaluation of methods for soil surface moisture estimation from reflectance data. International Journal of Remote Sensing, 24(10), 20692083.

57. Haubrock, S.N.; Chabrillat, S.; Lemmnitz, C.; Kaufmann, H. (2008). Surface soil moisture quantification models from reflectance data under field conditions. International Journal of Remote Sensing, 29(1), 3-29.

58. Lesaignoux, A.; Fabre, S.; Briottet, X. (2013). Influence of soil moisture content on spectral reflectance of bare soils in the $0.4-14 \mu \mathrm{m}$ domain. International Journal of Remote Sensing, 34(7), 2268-2285. 
59. Chappell, A.; Strong, C.; McTainsh, G.; Leys, J. (2007). Detecting induced in situ erodibility of a dust-producing playa in Australia using a bi-directional soil spectral reflectance model. Remote Sensing of Environment, 106(4), 508-524.

60. Chappell, A.; Zobeck, T.M.; Brunner, G. (2006). Using bi-directional soil spectral reflectance to model soil surface changes induced by rainfall and wind-tunnel abrasion. Remote Sensing of Environment, 102(3-4), 328-343.

61. Rosendahl, S.; Casselgren, J.; Sjödahl, M. (2013). Simplified model for light scattering from granular materials with varying moisture content. Applied Optics, 52(17), 4006-4012.

62. Hapke, B. (1981). Bidirectional reflectance spectroscopy. 1. Theory. J. Geophys. Res., 86(4 B), 3039-3054.

63. Pinty, B.; Verstraete, M.M.; Dickinson, R.E. (1989). A physical model for predicting bidirectional reflectances over bare soil. Remote Sensing of Environment, 27(3), 273-288.

64. Jacquemoud, S.; Baret, F.; Hanocq, J.F. (1992). Modeling spectral and bidirectional soil reflectance. Remote Sensing of Environment, 41(2-3), 123132.

65. Daughtry, C.S.T.; Hunt Jr, E.R. (2008). Mitigating the effects of soil and residue water contents on remotely sensed estimates of crop residue cover. Remote Sensing of Environment, 112(4), 1647-1657.

66. Rahman, H.; Pinty, B.; Verstraete, M.M. (1993). Coupled surfaceatmosphere reflectance (CSAR) model 2. Semiempirical surface model usable with NOAA advanced very high resolution radiometer data. Journal of Geophysical Research, 98(D11), 20,791-720,801.

67. Henyey, L.G.; Greenstein, J.L. (1941). Diffuse radiation in the galaxy. Astrophys. J., 93, 70-83.

68. Kay, S.; Hedley, J.D.; Lavender, S. (2009). Sun glint correction of high and low spatial resolution images of aquatic scenes: A review of methods for visible and near-infrared wavelengths. Remote Sensing, 1(4), 697-730.

69. Cox, C.; Munk, W. (1954). Measurement of the roughness of the sea surface from photographs of the sun's glitter. J. Opt. Soc. Am., 44(11), 838-850.

70. Oltra-Carrió, R.; Baup, F.; Fabre, S.; Fieuzal, R.; Briottet, X. (2015). Improvement of soil moisture retrieval from hyperspectral VNIR-SWIR data using clay content information: From laboratory to field experiments. Remote Sensing, 7(3), 3184-3205.

71. Weyermann, J.; Damm, A.; Kneubuhler, M.; Schaepman, M.E. (2014). Correction of reflectance anisotropy effects of vegetation on airborne spectroscopy data and derived products. IEEE Transactions on Geoscience and Remote Sensing, 52(1), 616-627. 
72. Lucht, W.; Schaaf, C.B.; Strahler, A.H. (2000). An algorithm for the retrieval of albedo from space using semiempirical BRDF models. IEEE Transactions on Geoscience and Remote Sensing, 38(2 II), 977-998.

73. Kneubühler, M.; Koetz, B.; Huber, S.; Schaepman, M.E.; Zimmermann, N.E. (2008). Space-based spectrodirectional measurements for the improved estimation of ecosystem variables. Canadian Journal of Remote Sensing, 34(3), 192-205.

74. Wang, Y.; Li, G.; Ding, J.; Guo, Z.; Tang, S.; Liu, R.; Chen, J. (2016). A combined GLAS and MODIS estimation of the global distribution of mean forest canopy height. Remote Sensing of Environment, 174, 24-43.

75. Chen, J.M.; Menges, C.H.; Leblanc, S.G. (2005). Global mapping of foliage clumping index using multi-angular satellite data. Remote Sensing of Environment, 97(4), 447-457.

76. He, L.; Chen, J.M.; Pisek, J.; Schaaf, C.B.; Strahler, A.H. (2012). Global clumping index map derived from the MODIS BRDF product. Remote Sensing of Environment, 119, 118-130.

77. Roosjen, P.P.J.; Bartholomeus, H.M.; Clevers, J.G.P.W. (2015). Effects of soil moisture content on reflectance anisotropy - Laboratory goniometer measurements and RPV model inversions. Remote Sensing of Environment, 170, 229-238.

78. Koukal, T.; Atzberger, C.; Schneider, W. (2014). Evaluation of semiempirical BRDF models inverted against multi-angle data from a digital airborne frame camera for enhancing forest type classification. Remote Sensing of Environment, 151, 27-43.

79. Bachmann, C.M.; Abelev, A.; Montes, M.J.; Philpot, W.; Gray, D.; Doctor, K.Z.; Fusina, R.A.; Mattis, G.; Chen, W.; Noble, S.D.; Coburn, C.; Corl, T.; Slomer, L.; Nichols, C.R.; Roggen, E.V.; Hughes, R.J.; Carr, S.; Kharabash, S.; Brady, A.; Vermillion, M. (2016). Flexible field goniometer system: The Goniometer for Outdoor Portable Hyperspectral Earth Reflectance. Journal of Applied Remote Sensing, 10(3).

80. Sandmeier, S.R.; Strahler, A.H. (2000). BRDF laboratory measurements. Remote Sensing Reviews, 18(2), 481-502.

81. Dangel, S.; Verstraete, M.M.; Schopfer, J.; Kneubühler, M.; Schaepman, M.; Itten, K.I. (2005). Toward a direct comparison of field and laboratory goniometer measurements. IEEE Transactions on Geoscience and Remote Sensing, 43(11), 2666-2675.

82. Milton, E.J.; Schaepman, M.E.; Anderson, K.; Kneubühler, M.; Fox, N. (2009). Progress in field spectroscopy. Remote Sensing of Environment, 113(SUPPL. 1), S92-S109.

83. Shahbazi, M.; Théau, J.; Ménard, P. (2014). Recent applications of unmanned aerial imagery in natural resource management. GIScience and Remote Sensing, 51(4), 339-365. 
84. Verger, A.; Vigneau, N.; Che'ron, C.; Gilliot, J.M.; Comar, A.; Baret, F. (2014). Green area index from an unmanned aerial system over wheat and rapeseed crops. Remote Sensing of Environment, 152, 654-664.

85. Gevaert, C.M.; Suomalainen, J.; Tang, J.; Kooistra, L. (2015). Generation of Spectral-Temporal Response Surfaces by Combining Multispectral Satellite and Hyperspectral UAV Imagery for Precision Agriculture Applications. IEEE Journal of Selected Topics in Applied Earth Observations and Remote Sensing, 8(6), 3140-3146.

86. Huang, Y.B.; Thomson, S.J.; Hoffmann, W.C.; Lan, Y.B.; Fritz, B.K. (2013). Development and prospect of unmanned aerial vehicle technologies for agricultural production management. International Journal of Agricultural and Biological Engineering, 6(3), 1-10.

87. Honkavaara, E.; Eskelinen, M.A.; Polonen, I.; Saari, H.; Ojanen, H.; Mannila, R.; Holmlund, C.; Hakala, T.; Litkey, P.; Rosnell, T.; Viljanen, N.; Pulkkanen, M. (2016). Remote Sensing of 3-D Geometry and Surface Moisture of a Peat Production Area Using Hyperspectral Frame Cameras in Visible to ShortWave Infrared Spectral Ranges Onboard a Small Unmanned Airborne Vehicle (UAV). IEEE Transactions on Geoscience and Remote Sensing, 54(9), 5440-5454.

88. Duan, S.B.; Li, Z.L.; Wu, H.; Tang, B.H.; Ma, L.; Zhao, E.; Li, C. (2014). Inversion of the PROSAIL model to estimate leaf area index of maize, potato, and sunflower fields from unmanned aerial vehicle hyperspectral data. International Journal of Applied Earth Observation and Geoinformation, 26(1), 12-20.

89. Rasmussen, J.; Ntakos, G.; Nielsen, J.; Svensgaard, J.; Poulsen, R.N.; Christensen, S. (2016). Are vegetation indices derived from consumergrade cameras mounted on UAVs sufficiently reliable for assessing experimental plots? European Journal of Agronomy, 74, 75-92.

90. Brede, B.; Suomalainen, J.; Bartholomeus, H.; Herold, M. (2015). Influence of solar zenith angle on the enhanced vegetation index of a Guyanese rainforest. Remote Sensing Letters, 6(12), 972-981.

91. Suomalainen, J.; Anders, N.; Iqbal, S.; Roerink, G.; Franke, J.; Wenting, P.; Hünniger, D.; Bartholomeus, H.; Becker, R.; Kooistra, L. (2014). A lightweight hyperspectral mapping system and photogrammetric processing chain for unmanned aerial vehicles. Remote Sensing, 6(11), 11013-11030.

92. Verrelst, J.; Clevers, J.G.P.W.; Schaepman, M.E. (2010). Merging the Minnaert-k parameter with spectral unmixing to map forest heterogeneity with CHRIS/PROBA data. IEEE Transactions on Geoscience and Remote Sensing, 48(11), 4014-4022.

93. Widlowski, J.L.; Pinty, B.; Gobron, N.; Verstraete, M.M.; Diner, D.J.; Davis, A.B. (2004). Canopy structure parameters derived from multi-angular remote sensing data for terrestrial carbon studies. Climatic Change, 67(23), 403-415. 
94. Wassenaar, T.; Andrieux, P.; Baret, F.; Robbez-Masson, J.M. (2005). Soil surface infiltration capacity classification based on the bi-directional reflectance distribution function sampled by aerial photographs. The case of vineyards in a Mediterranean area. Catena, 62(2-3), 94-110.

95. Biliouris, D.; van der Zande, D.; Verstraeten, W.W.; Stuckens, J.; Muys, B.; Dutré, P.; Coppin, P. (2009). RPV model parameters based on hyperspectral bidirectional reflectance measurements of Fagus sylvatica L. Leaves. Remote Sensing, 1(2), 92-106.

96. R Core Team (2016). R: A Language and Environment for Statistical Computing. R Foundation for Statistical Computing: Vienna, Austria.

97. Breunig, F.M.; Galvão, L.S.; Formaggio, A.R.; Epiphanio, J.C.N. (2011). Directional effects on NDVI and LAI retrievals from MODIS: A case study in Brazil with soybean. International Journal of Applied Earth Observation and Geoinformation, 13(1), 34-42.

98. Alonso, L.; Moreno, J.; Leroy, M. (2001). BRDF signatures from polder data. In: European Space Agency, (Special Publication) ESA SP, 2001; p 183.

99. Jackson, R.D.; Teillet, P.M.; Slater, P.N.; Fedosejevs, G.; Jasinski, M.F.; Aase, J.K.; Moran, M.S. (1990). Bidirectional measurements of surface reflectance for view angle corrections of oblique imagery. Remote Sensing of Environment, 32(2-3), 189-202.

100. Zhou, K.; Guo, Y.; Geng, Y.; Zhu, Y.; Cao, W.; Tian, Y. (2014). Development of a novel bidirectional canopy reflectance model for row-planted rice and wheat. Remote Sensing, 6, 7632-7659.

101. Huang, W.; Wang, Z.; Huang, L.; Lamb, D.W.; Ma, Z.; Zhang, J.; Wang, J.; Zhao, C. (2011). Estimation of vertical distribution of chlorophyll concentration by bi-directional canopy reflectance spectra in winter wheat. Precision Agriculture, 12(2), 165-178.

102. Sun, T.; Fang, H.; Liu, W.; Ye, Y. (2017). Impact of water background on canopy reflectance anisotropy of a paddy rice field from multi-angle measurements. Agricultural and Forest Meteorology, 233, 143-152.

103. Hill, M.J.; Averill, C.; Jiao, Z.; Schaaf, C.B.; Armston, J.D. (2008). Relationship of MISR RPV parameters and MODIS BRDF shape indicators to surface vegetation patterns in an Australian tropical savanna. Canadian Journal of Remote Sensing, 34(SUPPL. 2), S247-S267.

104. Lavergne, T.; Kaminski, T.; Pinty, B.; Taberner, M.; Gobron, N.; Verstraete, M.M.; Vossbeck, M.; Widlowski, J.L.; Giering, R. (2007). Application to MISR land products of an RPV model inversion package using adjoint and Hessian codes. Remote Sensing of Environment, 107(1-2), 362-375.

105. Bousquet, L.; Lachérade, S.; Jacquemoud, S.; Moya, I. (2005). Leaf BRDF measurements and model for specular and diffuse components differentiation. Remote Sensing of Environment, 98(2-3), 201-211. 
106. Huang, W.; Niu, Z.; Wang, J.; Liu, L.; Zhao, C.; Liu, Q. (2006). Identifying crop leaf angle distribution based on two-temporal and bidirectional canopy reflectance. IEEE Transactions on Geoscience and Remote Sensing, 44(12), 3601-3608.

107. Roujean, J.L.; Leroy, M.; Deschamps, P.Y. (1992). A bidirectional reflectance model of the Earth's surface for the correction of remote sensing data. Journal of Geophysical Research, 97(D18), 20,455-420,468.

108. Brown De Colstoun, E.C.; Walthall, C.L. (2006). Improving global scale land cover classifications with multi-directional POLDER data and a decision tree classifier. Remote Sensing of Environment, 100(4), 474-485.

109. Duca, R.; Del Frate, F. (2008). Hyperspectral and multiangle CHRIS-PROBA images for the generation of land cover maps. IEEE Transactions on Geoscience and Remote Sensing, 46(10), 2857-2866.

110. Su, L.; Huang, Y.; Chopping, M.J.; Rango, A.; Martonchik, J.V. (2009). An empirical study on the utility of BRDF model parameters and topographic parameters for mapping vegetation in a semi-arid region with MISR imagery. International Journal of Remote Sensing, 30(13), 3463-3483.

111. Wang, L.; Dong, T.; Zhang, G.; Niu, Z. (2013). LAI retrieval using PROSAIL model and optimal angle combination of multi-angular data in wheat. IEEE Journal of Selected Topics in Applied Earth Observations and Remote Sensing, 6(3), 1730-1736.

112. Painter, T.H.; Dozier, J. (2004). Measurements of the hemisphericaldirectional reflectance of snow at fine spectral and angular resolution. Journal of Geophysical Research Atmospheres, 109(18), D18115 1811118121.

113. Peltoniemi, J.I.; Kaasalainen, S.; Näränen, J.; Matikainen, L.; Piironen, J. (2005). Measurement of directional and spectral signatures of light reflectance by snow. IEEE Transactions on Geoscience and Remote Sensing, 43(10), 2294-2304.

114. Miller, I.; Forster, B.C.; Laffan, S.W.; Brander, R.W. (2016). Bidirectional reflectance of coral growth-forms. International Journal of Remote Sensing, 37(7), 1553-1567.

115. Roosjen, P.P.J.; Suomalainen, J.M.; Bartholomeus, H.M.; Clevers, J.G.P.W. (2016). Hyperspectral reflectance anisotropy measurements using a pushbroom spectrometer on an unmanned aerial vehicle-Results for barley, winter wheat, and potato. Remote Sensing, 8(11), 909.

116. Näsi, R.; Honkavaara, E.; Lyytikäinen-Saarenmaa, P.; Blomqvist, M.; Litkey, P.; Hakala, T.; Viljanen, N.; Kantola, T.; Tanhuanpää, T.; Holopainen, M. (2015). Using UAV-based photogrammetry and hyperspectral imaging for mapping bark beetle damage at tree-level. Remote Sensing, 7(11), 1546715493. 
117. Stoorvogel, J.J.; Kooistra, L.; Bouma, J. (2015). Managing soil variability at different spatial scales as a basis for precision agriculture. Advances in Soil Science, 37-72.

118. Clevers, J.G.P.W. (1989). Application of a weighted infrared-red vegetation index for estimating leaf Area Index by Correcting for Soil Moisture. Remote Sensing of Environment, 29(1), 25-37.

119. Kooistra, L.; Clevers, J.G.P.W. (2016). Estimating potato leaf chlorophyll content using ratio vegetation indices. Remote Sensing Letters, 7(6), 611620.

120. Drusch, M.; Del Bello, U.; Carlier, S.; Colin, O.; Fernandez, V.; Gascon, F.; Hoersch, B.; Isola, C.; Laberinti, P.; Martimort, P.; Meygret, A.; Spoto, F.; Sy, O.; Marchese, F.; Bargellini, P. (2012). Sentinel-2: ESA's Optical HighResolution Mission for GMES Operational Services. Remote Sensing of Environment, 120, 25-36.

121. Honkavaara, E.; Saari, H.; Kaivosoja, J.; Pölönen, I.; Hakala, T.; Litkey, P.; Mäkynen, J.; Pesonen, L. (2013). Processing and assessment of spectrometric, stereoscopic imagery collected using a lightweight UAV spectral camera for precision agriculture. Remote Sensing, 5(10), 50065039.

122. Maignan, F.; Bréon, F.M.; Lacaze, R. (2004). Bidirectional reflectance of Earth targets: Evaluation of analytical models using a large set of spaceborne measurements with emphasis on the Hot Spot. Remote Sensing of Environment, 90(2), 210-220.

123. Obidiegwu, J.E.; Bryan, G.J.; Jones, H.G.; Prashar, A. (2015). Coping with drought: Stress and adaptive responses in potato and perspectives for improvement. Frontiers in Plant Science, 6(542), 1-23.

124. Schlapfer, D.; Richter, R.; Feingersh, T. (2015). Operational BRDF effects correction for wide-field-of-view optical scanners (BREFCOR). IEEE Transactions on Geoscience and Remote Sensing, 53(4), 1855-1864.

125. Honkavaara, E.; Hakala, T.; Nevalainen, O.; Viljanen, N.; Rosnell, T.; Khoramshahi, E.; Näsi, R.; Oliveira, R.; Tommaselli, A. (2016). Geometric and reflectance signature characterization of complex canopies using hyperspectral stereoscopic images from uav and terrestrial platforms. In: International Archives of the Photogrammetry, Remote Sensing and Spatial Information Sciences - ISPRS Archives, 2016; pp 77-82.

126. Verrelst, J.; Romijn, E.; Kooistra, L. (2012). Mapping vegetation density in a heterogeneous river floodplain ecosystem using pointable CHRIS/PROBA data. Remote Sensing, 4(9), 2866-2889.

127. Jacquemoud, S.; Verhoef, W.; Baret, F.; Bacour, C.; Zarco-Tejada, P.J.; Asner, G.P.; François, C.; Ustin, S.L. (2009). PROSPECT + SAIL models: A review of use for vegetation characterization. Remote Sensing of Environment, 113(SUPPL. 1), S56-S66. 
128. Domingues Franceschini, M.; Bartholomeus, H.; van Apeldoorn, D.; Suomalainen, J.; Kooistra, L. (2017). Intercomparison of Unmanned Aerial Vehicle and Ground-Based Narrow Band Spectrometers Applied to Crop Trait Monitoring in Organic Potato Production. Sensors, 17(6), 1428.

129. Jay, S.; Maupas, F.; Bendoula, R.; Gorretta, N. (2017). Retrieving LAI, chlorophyll and nitrogen contents in sugar beet crops from multi-angular optical remote sensing: Comparison of vegetation indices and PROSAIL inversion for field phenotyping. Field Crops Research, 210, 33-46.

130. Cilia, C.; Panigada, C.; Rossini, M.; Meroni, M.; Busetto, L.; Amaducci, S.; Boschetti, M.; Picchi, V.; Colombo, R. (2014). Nitrogen status assessment for variable rate fertilization in maize through hyperspectral imagery. Remote Sensing, 6(7), 6549-6565.

131. Goffart, J.P.; Olivier, M.; Frankinet, M. (2008). Potato crop nitrogen status assessment to improve $\mathrm{N}$ fertilization management and efficiency: Pastpresent-future. Potato Research, 51(3-4), 355-383.

132. Jacquemoud, S.; Bacour, C.; Poilvé, H.; Frangi, J.P. (2000). Comparison of four radiative transfer models to simulate plant canopies reflectance: Direct and inverse mode. Remote Sensing of Environment, 74(3), 471-481.

133. Jacquemoud, S.; Baret, F. (1990). PROSPECT: A model of leaf optical properties spectra. Remote Sensing of Environment, 34(2), 75-91.

134. Verhoef, W. (1984). Light scattering by leaf layers with application to canopy reflectance modeling: The SAIL model. Remote Sensing of Environment, 16(2), 125-141.

135. Darvishzadeh, R.; Skidmore, A.; Schlerf, M.; Atzberger, C. (2008). Inversion of a radiative transfer model for estimating vegetation LAI and chlorophyll in a heterogeneous grassland. Remote Sensing of Environment, 112(5), 2592-2604.

136. Combal, B.; Baret, F.; Weiss, M.; Trubuil, A.; Macé, D.; Pragnère, A.; Myneni, R.; Knyazikhin, Y.; Wang, L. (2003). Retrieval of canopy biophysical variables from bidirectional reflectance using prior information to solve the ill-posed inverse problem. Remote Sensing of Environment, 84(1), 1-15.

137. Jacquemoud, S.; Baret, F.; Andrieu, B.; Danson, F.M.; Jaggard, K. (1995). Extraction of vegetation biophysical parameters by inversion of the PROSPECT + SAIL models on sugar beet canopy reflectance data. Application to TM and AVIRIS sensors. Remote Sensing of Environment, 52(3), 163-172.

138. Weiss, M.; Baret, F. (1999). Evaluation of canopy biophysical variable retrieval performances from the accumulation of large swath satellite data. Remote Sensing of Environment, 70(3), 293-306.

139. Laurent, V.C.E.; Schaepman, M.E.; Verhoef, W.; Weyermann, J.; Chávez, R.O. (2014). Bayesian object-based estimation of LAI and chlorophyll from a simulated Sentinel-2 top-of-atmosphere radiance image. Remote Sensing of Environment, 140, 318-329. 
140. Mousivand, A.; Menenti, M.; Gorte, B.; Verhoef, W. (2015). Multi-temporal, multi-sensor retrieval of terrestrial vegetation properties from spectraldirectional radiometric data. Remote Sensing of Environment, 158, 311-330.

141. Dorigo, W.A. (2012). Improving the robustness of cotton status characterisation by radiative transfer model inversion of multi-angular CHRIS/PROBA data. IEEE Journal of Selected Topics in Applied Earth Observations and Remote Sensing, 5(1), 18-29.

142. Schaepman, M.E.; Koetz, B.; Schaepman-Strub, G.; Itten, K.I. (2005). Spectrodirectional remote sensing for the improved estimation of biophysical and -chemical variables: Two case studies. International Journal of Applied Earth Observation and Geoinformation, 6(3-4), 271-282.

143. Vuolo, F.; Dini, L.; D'Urso, G. (2008). Retrieval of leaf area index from CHRIS/PROBA data: An analysis of the directional and spectral information content. International Journal of Remote Sensing, 29(17-18), 5063-5072.

144. Weiss, M.; Baret, F.; Myneni, R.B.; Pragnère, A.; Knyazikhin, Y. (2000). Investigation of a model inversion technique to estimate canopy biophysical variables from spectral and directional reflectance data. Agronomie, 20(1), 3-22.

145. Barnsley, M.J.; Strahler, A.H.; Morris, K.P.; Muller, J.P. (1994). Sampling the surface bidirectional reflectance distribution function (BRDF): 1. evaluation of current and future satellite sensors. Remote Sensing Reviews, $8(4), 271-311$.

146. Chen, J.M.; Liu, J.; Leblanc, S.G.; Lacaze, R.; Roujean, J.L. (2003). Multiangular optical remote sensing for assessing vegetation structure and carbon absorption. Remote Sensing of Environment, 84(4), 516-525.

147. Sandmeier, S.R. (2000). Acquisition of bidirectional reflectance factor data with field goniometers. Remote Sensing of Environment, 73(3), 257-269.

148. Roosjen, P.P.J.; Suomalainen, J.S.; Bartholomeus, H.M.; Kooistra, L.K.; Clevers, J.G.P.W. (2017). Mapping reflectance anisotropy of a potato canopy using aerial images acquired with an unmanned aerial vehicle. Remote Sensing, 9(5), 417.

149. Clevers, J.; Kooistra, L.; van den Brande, M. (2017). Using Sentinel-2 Data for Retrieving LAI and Leaf and Canopy Chlorophyll Content of a Potato Crop. Remote Sensing, 9(5), 405.

150. Schopfer, J.; Dangel, S.; Kneubühler, M.; Itten, K.I. (2008). The improved dual-view field goniometer system FIGOS. Sensors, 8(8), 5120-5140.

151. Lehnert, L.W.; Meyer, H.; Bendix, J. (2016). hsdar: Manage, analyse and simulate hyperspectral data in $\mathrm{R}$.

152. Casa, R.; Jones, H.G. (2004). Retrieval of crop canopy properties: A comparison between model inversion from hyperspectral data and image classification. International Journal of Remote Sensing, 25(6), 1119-1130. 
153. Baret, F.; Hagolle, O.; Geiger, B.; Bicheron, P.; Miras, B.; Huc, M.; Berthelot, B.; Niño, F.; Weiss, M.; Samain, O.; Roujean, J.L.; Leroy, M. (2007). LAI, fAPAR and fCover CYCLOPES global products derived from VEGETATION. Part 1: Principles of the algorithm. Remote Sensing of Environment, 110(3), 275286.

154. Koetz, B.; Baret, F.; Poilvé, H.; Hill, J. (2005). Use of coupled canopy structure dynamic and radiative transfer models to estimate biophysical canopy characteristics. Remote Sensing of Environment, 95(1), 115-124.

155. Richter, K.; Atzberger, C.; Vuolo, F.; Weihs, P.; D'Urso, G. (2009). Experimental assessment of the Sentinel-2 band setting for RTM-based LAI retrieval of sugar beet and maize. Canadian Journal of Remote Sensing, 35(3), 230-247.

156. Verrelst, J.; Rivera, J.P.; Leonenko, G.; Alonso, L.; Moreno, J. (2014). Optimizing LUT-based RTM inversion for semiautomatic mapping of crop biophysical parameters from sentinel-2 and -3 data: Role of cost functions. IEEE Transactions on Geoscience and Remote Sensing, 52(1), 257-269.

157. Baret, F.; Buis, S. (2008). Estimating canopy characteristics from remote sensing observations: Review of methods and associated problems. In: Advances in Land Remote Sensing: System, Modeling, Inversion and Application, 2008; pp 173-201.

158. Botha, E.J.; Leblon, B.; Zebarth, B.; Watmough, J. (2007). Non-destructive estimation of potato leaf chlorophyll from canopy hyperspectral reflectance using the inverted PROSAIL model. International Journal of Applied Earth Observation and Geoinformation, 9(4), 360-374.

159. Qi, J.; Cabot, F.; Moran, M.S.; Dedieu, G. (1995). Biophysical parameter estimations using multidirectional spectral measurements. Remote Sensing of Environment, 54(1), 71-83.

160. Nouwakpo, S.K.; Weltz, M.A.; McGwire, K. (2016). Assessing the performance of structure-from-motion photogrammetry and terrestrial LiDAR for reconstructing soil surface microtopography of naturally vegetated plots. Earth Surface Processes and Landforms, 41(3), 308-322.

161. Snapir, B.; Hobbs, S.; Waine, T.W. (2014). Roughness measurements over an agricultural soil surface with Structure from Motion. ISPRS Journal of Photogrammetry and Remote Sensing, 96, 210-223.

162. Liang, S.; Strahler, A.H.; Barnsley, M.J.; Borel, C.C.; Gerstl, S.A.W.; Diner, D.J.; Prata, A.J.; Walthall, C.L. (2000). Multiangle remote sensing: Past, present and future. Remote Sensing Reviews, 18(2), 83-102.

163. Mallet, C.; Bretar, F. (2009). Full-waveform topographic lidar: State-of-theart. ISPRS Journal of Photogrammetry and Remote Sensing, 64(1), 1-16.

164. Hunt, E.R.; Horneck, D.A.; Spinelli, C.B.; Turner, R.W.; Bruce, A.E.; Gadler, D.J.; Brungardt, J.J.; Hamm, P.B. (2017). Monitoring nitrogen status of potatoes using small unmanned aerial vehicles. Precision Agriculture, 1-20. 
165. Torres-Sánchez, J.; Peña, J.M.; de Castro, A.I.; López-Granados, F. (2014). Multi-temporal mapping of the vegetation fraction in early-season wheat fields using images from UAV. Computers and Electronics in Agriculture, 103, 104-113.

166. Matese, A.; Toscano, P.; Di Gennaro, S.F.; Genesio, L.; Vaccari, F.P.; Primicerio, J.; Belli, C.; Zaldei, A.; Bianconi, R.; Gioli, B. (2015). Intercomparison of UAV, aircraft and satellite remote sensing platforms for precision viticulture. Remote Sensing, 7(3), 2971-2990.

167. Verrelst, J.; Camps-Valls, G.; Muñoz-Marí, J.; Rivera, J.P.; Veroustraete, F.; Clevers, J.G.P.W.; Moreno, J. (2015). Optical remote sensing and the retrieval of terrestrial vegetation bio-geophysical properties - A review. ISPRS Journal of Photogrammetry and Remote Sensing, 108, 273-290. 


\section{Summary}

Optical remote sensing is a well-established technology for qualitative and quantitative characterization of the Earth's surface. Using optical remote sensing techniques, biophysical crop parameters can be estimated based on the reflected radiation either through empirical-statistical methods, or through inversion of radiative transfer models. Natural surfaces, however, reflect light anisotropically, which means that the intensity of reflected light depends on the viewing and illumination geometry. On the one hand, reflectance anisotropy is considered as an unwanted effect that needs to be corrected for, since it leads to inaccuracies in parameter estimations. On the other hand, reflectance anisotropy can be considered as a source of information in addition to spectral data, due to its unique response to optical and structural properties of the observed surface.

For several decades, reflectance anisotropy has been studied using ground-based and space-borne sensors that are capable of collecting multi-angular measurements. Both ground-based and space-borne platforms have their own specific advantages and shortcomings. In the last few years, the opportunities of Unmanned Aerial Vehicles (UAVs) to collect multi-angular measurements started to be explored. Due to the relatively low flight height of these platforms, UAVs have the potential to study reflectance anisotropy at high spatial resolution, which is needed for applications such as precision agriculture. Until now, the attempts to collect multi-angular data using UAVS is rather limited. The main objectives of this thesis were (i) to study the relation between reflectance anisotropy and properties of crops and soils that are important for agriculture; (ii) to study the ability of UAVs to collect multi-angular measurements; (iii) to study the use of multi-angular observations to improve estimations of crop parameters.

In chapter 2, the effects of soil moisture content (SMC), an important environmental parameter for agriculture, on reflectance anisotropy have been studied. Optical remote sensing methods to study SMC are often based on empirically or physically based models that relate changes in reflectance intensity to SMC. The effects of SMC on the reflectance anisotropy, however, have not received much attention. Using a laboratory goniometer setup, multi-angular reflectance measurements were taken of five soil samples during a dry-out experiment. At the start of the experiment, the soil samples were saturated with water and placed in the center of the goniometer. Reflectance measurements in the $350-2500 \mathrm{~nm}$ range were taken continuously at 60 positions around the target, covering the full hemisphere, while the soil samples were left to dry. The RahmanPinty-Verstraete (RPV) model, a semi-empirical model that is able to describe the reflectance anisotropy of any arbitrary surface using just four parameters, was fitted though the multi-angular measurements to parameterize and quantify the reflectance anisotropy of the soil samples at different SMC levels during the dry-out 
experiment. The results of this study showed that SMC has a strong influence on reflectance anisotropy. Moreover, studying the relation between the estimated RPV parameters and the SMC level revealed a strong relation between SMC and the parameter responsible for the forward / backward scattering intensity. This relation was even stronger than the relation between the parameter that relates to the nadir reflectance and SMC, indicating that the anisotropy signal contains important information on SMC.

Although the goniometer that was used to perform the measurements of chapter 2 is highly suitable for measurements of flat targets such as soils, it is unsuitable for measurements of objects that have a strong three dimensional structure, such as vegetation or crops. Chapter 3 explored the potential of using an unmanned aerial vehicle (UAV) to perform multi-angular measurements of such targets. The used equipment for the measurements consisted of an Altura AT8 octocopter with a downward-pointing hyperspectral line scanner (450-915 nm at a 5-nm sampling interval) mounted underneath it; a configuration that is typically used for mapping of small areas of 2-10 ha. By hovering the UAV at a relatively low height above a canopy, while rotating it around its vertical axis, we were able to capture the reflectance anisotropy of a barley, potato, and winter wheat canopy. The reflectance anisotropy was again quantified and parameterized using the RPV model. The experiment was repeated twice during the growing season of 2015, which enabled the study of the effects of canopy development for the barley, potato, and winter wheat canopies on reflectance anisotropy. The results of this repetition showed that canopy development for barley and winter wheat (the development of grain heads) resulted in significant changes in the observed anisotropy signal. For the potato canopy, where the canopy developed from an open row-structure to a closed canopy between the two measurement days, there was a clear reduction of the anisotropy. Especially, the backward scattering intensity had reduced over the whole measured spectrum. The results demonstrated that anisotropy can be measured with a line scanner mounted under a UAV, and moreover, the results showed that canopy development can be observed in the anisotropy signal.

The method used in chapter 3 provides an average anisotropy signal of the scanned area. However, it does not provide any information on the spatial distribution or the heterogeneity of the anisotropy within the field. In chapter 4 , we used a snapshot camera (500-900 $\mathrm{nm}$ ) mounted on a UAV to capture pixel-wise anisotropy. Using the location of the georeferenced pixels after photogrammetric processing of the collected imagery, combined with the location of the UAV during the image acquisition, it was possible to calculate the viewing geometry per pixel. In areas where the collected imagery overlapped, pixels were thus captured from multiple view angles, resulting in a multi-angular dataset for each pixel. The RPV model was used to quantify and parameterize the observed anisotropy per pixel and RPV-parameter maps were created, showing the spatial distribution of the anisotropy in the area covered by the UAV. For this chapter, data were collected over a potato field where a fertilizer experiment was performed. Different initial fertilization levels (before planting) in specific zones in the field and sensor-based additional fertilization applied to half of the field during the growing season resulted in variation in the growth of the potato plants, and therefore in differences 
in canopy cover and leaf area index (LAI). A correlation study between the RPV parameters and measurements in the field of LAI and estimations of canopy cover based on high resolution imagery showed that there was a strong relation between the RPV parameters and these canopy properties, indicating that there is information on LAI and canopy cover in the anisotropy signal. Moreover, this study showed that it is relatively easy to collect multi-angular measurements using a snapshot camera mounted on a UAV.

In chapter 5, we used the multi-angular dataset of chapter 4 to study the use of multi-angular observations for the improvement of estimations of vegetation parameters. We numerically inverted the PROSAIL model to estimate LAI and leaf chlorophyll content (LCC) based on multi-angular data and compared the accuracy of the estimations to inversion of the PROSAIL model based on nadir data, with in situ measurements of LAI and LCC as a reference. Moreover, we repeated the same exercise for several simulated multi-angular datasets with angular configurations that were captured during the UAV flight, and angular configurations that are typically captured by goniometer setups. The results showed that for the simulated datasets, strong improvements in the estimation of LAI and LCC could be obtained when multi-angular data were used for inversion, compared to when nadir data were used. For the measured dataset also improvements were found when multiangular data were used for inversion, however, not as large as with the simulated data. The results of this study indicate that when the used model is capable of reproducing the input data (as was the case for the simulated data), estimates of LAI and LCC can be improved significantly. Moreover, the results indicated that multi-angular views collected in overlapping pixels that are captured by snapshot cameras during a typical UAV mapping flight already provide the angular coverage needed to improve inversion results.

In chapter 6, the main findings of this thesis were discussed. The overall conclusion is that multi-angular reflectance data can improve the estimation of crop parameters and, moreover, that UAVs are highly capable platforms to perform these multi-angular measurements. The fine spatial resolution that is typically obtained in UAV-based measurements provides a new intermediate measurement scale for the study of reflectance anisotropy compared to the relatively coarse spatial resolution measurements obtained by satellite-based sensors and the point data acquired by ground-based goniometers. The results presented in this thesis provide a good starting point for reflectance anisotropy research with UAVs and the further development of methods to improve crop parameter retrieval using multiangular observations. 


\section{Acknowledgements}

First of all, I would like to thank my promotor Martin for giving me the opportunity to do my PhD. Also, I would like to acknowledge Jan and Harm, who dared to supervise me again after already having supervised me during my master's thesis. Thanks for always helping me when needed and for giving me the space and freedom to carry out my own research.

Juha, I very much appreciate all your help with the UAV field work and data processing. I have learned a lot from you and without your effort I probably would not have made it so far. Benjamin, thanks for helping me out with my programming issues and the writing of my last manuscript. Lammert, also you I want to thank for your comments and suggestions for my manuscripts, and for providing me the opportunity to do a postdoc and stick around a bit longer in Wageningen.

I had a great time during my $\mathrm{PhD}$, mainly due to all the nice people that work in our department: Aldo, Sytze, Arnold, Willy, Lammert, Ron, Marcello, Arend, Frans, John, Devis, Jan, Corné, Roland, and not to forget Antoinette and Truus. Further, I also want to thank all the other PhD candidates and postdocs: Ben, Eskender, Mathieu, Sarah, Kim, Johannes, Jose, Nandika, Michi, Giulia, Richard, Niki, Arun, Patric, Roberto, Brice, Erika, Kalkidan, Astrid, Yang, Simon, Tsoefiet, Valerio, Rosa, Alvaro, Qijun, Maria, Marston, Konstantin, Sabina, Marian, Jalal, Danaë, Joao, Daniela, Eliakim, and Benjamin. A special thanks of course to Kim, Loïc, Johannes, and Dirk, with whom I have shared an office throughout my PhD.

I would like to thank all my sporty friends, Juha, Lukasz, and Mathieu for all the nice weekly squash games and beers. En natuurlijk ook mijn volleybal team: Bob, Dennis, Jochem, Sipke, Gijs, Erik, Karst, Rik, Kris, Roel, Kees, en Gerald voor het mooie seizoen met als afsluiting een glorieus kampioenschap.

There is no productive day without a game of poker before, during, or after the break. Therefore, thanks Mathieu, Michi, Richard, and Simon for the many inspiring poker sessions. We should seriously get a new deck of cards!

Although Wageningen is quite small, thanks to Jelke and Annelien, Jeroen and Catalina, Sander and Amaya, Mia and Jeroen, Paolo and Sui there was always something to do.

Er is niks mooiers dan een weekend ouderwets gamen en bier drinken met de lannerds: Jorick, Jelmer, Sierd, Dennis, Paul L., Paul R., Lobke, Per, en Wim. Met name dank aan degene die elke jaar weer hun huis opofferen om deze sociale gelegenheid te hosten. De lan traditie houd al vele jaren stand en ik hoop dat dit in de toekomst nog lang zal blijven bestaan. 
Muchas gracias a los ticos, Carlos and Carla, Rafael and Marcela, Sergio and Madeline, Coen and Xiomara, Irene, Fran, and Vicky and Pieter for the many nice dinners, Sinterklaas celebrations, and Kerstmis games.

Muchas gracias a Angelina and the rest of the tico family. I'm very happy to have another home on the other side of the world and I hope to continue visiting you as much as possible! Also gracias a Jorge and family for your hospitality and showing me around in Miami.

Verder wil ik ook uiteraard de Wajo's bedanken. Arjen, Bas, Jelke, Jur, Paul, en Sander, mooi dat we na al die tijd nog regelmatig samen komen en jaarlijks hoogstaande culturele uitstapjes maken.

Jelke en Jeroen, wat een mooie tijd was dat aan het begin van mijn PhD. Dagelijks pompen in de pauze en 's avonds biertjes in de stad. We hebben vele mooie uitstapjes gemaakt, van snorkelen in de Thaise wateren, tot 696-en in de Zwitserse Alpen, en stuiteren over de muertos in Costa Rica. Jullie twee als mijn paranimfen is een mooie manier om deze periode af te sluiten.

Pa en ma, bedankt dat jullie mij altijd gesteund hebben, niet alleen tijdens mijn PhD, maar ook tijdens mijn studie periode ervoor. Jullie deur stond -en staat gelukkig nog steeds- altijd open. Ik kon altijd bij jullie binnenvallen om een hapje mee te eten na mijn werk, en als ik de bus had gemist om naar het vliegveld te komen waren jullie gek genoeg om mij met de auto te brengen zodat ik mijn vlucht niet zou missen. Heel erg bedankt daarvoor! Marieke, Paul en Lobke, gezellig dat we met z'n allen uiteindelijk in Wageningen zijn geëindigd en elkaar nog vaak kunnen zien. Thanks Paul dat ik altijd je auto kon lenen!

En tot slot natuurlijk de belangrijkste van allemaal: Marcela. Met jouw eindeloze energie was het geen moment stil de afgelopen jaren. Het dagelijkse ontbijt op bed met jou was en is de perfecte start van elke dag. Nu dat jij ook een postdoc gaat doen in Wageningen kunnen we de aankomende jaren gezellig elke dag naar het werk blijven fietsen. Ik kijk er naar uit om samen de vele reizen die we gepland hadden voor 'als we klaar zijn met onze PhD' te ondernemen! 


\section{List of publications}

\section{Peer reviewed publications}

Roosjen, P.P.J.; Brede, B.; Suomalainen, J.S.; Bartholomeus, H.M.; Kooistra, L.; Clevers, J.G.P.W. Improved estimation of leaf area index and leaf chlorophyll content using multi-angle spectral data collected by an unmanned aerial vehicle. Under review at the International Journal of Applied Earth Observation and Geoinformation.

Fernandez-Gutierrez, M.M.; Roosjen, P.P.J.; Ultee, E.; Agelink, M.; Vervoort, J.J.M.; Keijser, B.; Wells, J.M.; Kleerebezem, M. (2017). Streptococcus salivarius MS-oralD6 promotes gingival re-epithelialization in vitro through a secreted serine protease. Scientific Reports, 7(1), 11100.

Roosjen, P.P.J.; Suomalainen, J.S.; Bartholomeus, H.M.; Kooistra, L.; Clevers, J.G.P.W. (2017). Mapping reflectance anisotropy of a potato canopy using aerial images acquired with an unmanned aerial vehicle. Remote Sensing, 9(5), 417.

Roosjen, P.P.J.; Suomalainen, J.M.; Bartholomeus, H.M.; Clevers, J.G.P.W. (2016). Hyperspectral reflectance anisotropy measurements using a pushbroom spectrometer on an unmanned aerial vehicle-Results for barley, winter wheat, and potato. Remote Sensing, 8(11), 909.

Roosjen, P.P.J.; Bartholomeus, H.M.; Clevers, J.G.P.W. (2015). Effects of soil moisture content on reflectance anisotropy-Laboratory goniometer measurements and RPV model inversions. Remote Sensing of Environment, 170, 229-238.

Fros, J.J.; Geertsema, C.; Vogels, C.B.; Roosjen, P.P.J.; Failloux, A.B.; Vlak, J.M.; Koenraadt, C.J.; Takken, W.; Pijlman, G.P. (2015). West Nile virus: High transmission rate in north-western european mosquitoes indicates its epidemic potential and warrants increased surveillance. PLoS Neglected Tropical Diseases, 9(7), 1-12.

Nolet, C.; Poortinga, A.; Roosjen, P.P.J.; Bartholomeus, H.M.; Ruessink, G. (2014). Measuring and modeling the effect of surface moisture on the spectral reflectance of coastal beach sand. PLoS ONE, 9(11), e112151.

Roosjen, P.P.J.; Clevers, J.G.P.W.; Bartholomeus, H.M.; Schaepman, M.E.; Schaepman-Strub, G.; Jalink, H.; van der Schoor, R.; de Jong, A. (2012). A laboratory goniometer system for measuring reflectance and emittance anisotropy. Sensors, 12(12), 17358-17371. 


\section{Other scientific contributions}

Roosjen, P.P.J.; Suomalainen, J.M.; Bartholomeus, H.M.; Clevers, J.G.P.W. (2016). UAV-based reflectance anisotropy measurements of an experimental potato field. In: ESA Living Planet Symposium, Prague, Czech Republic, 9-13 May 2016.

Roosjen, P.P.J.; Bartholomeus, H.M.; Clevers, J.G.P.W. (2016). Effects of soil moisture content on reflectance anisotropy. In: ESA Living Planet Symposium, Prague, Czech Republic, 9-13 May 2016.

Roosjen, P.P.J.; Bartholomeus, H.M.; Suomalainen, J.M.; Clevers, J.G.P.W. (2015). Capturing Spatially Explicit Anisotropy Effects using UAV Based Multi-angular Reflectance Measurements. In: Earth Observation Science \& Society Symposium, Den Hague, The Netherlands, 1 October 2015.

Suomalainen, J.S.; Roosjen, P.P.J.; Bartholomeus, H.M.; Clevers, J.G.P.W. (2015). Reflectance anisotropy measurements using a pushbroom spectrometer mounted on a UAV and laboratory goniometer-Preliminary results. In: International Archives of the Photogrammetry, Remote Sensing and Spatial Information Sciences ISPRS Archives, Toronto, Canada, 30 August - 2 September 2015; pp 257-259.

Roosjen, P.P.J.; Bartholomeus, H.M.; Suomalainen, J.M.; Clevers, J.G.P.W. (2015). Investigating BRDF effects based on optical multi-angular laboratory and hyperspectral UAV measurements. In: 9th EARSeL Imaging Spectroscopy Workshop, Luxembourg, Luxembourg, 14-16 April 2015.

Roosjen, P.P.J.; Bartholomeus, H.M.; Suomalainen, J.M.; Clevers, J.G.P.W. (2015). Investigating BRDF effects based on optical multi-angular laboratory and hyperspectral UAV measurements. In: Fourier Transform Spectroscopy and Hyperspectral Imaging and Sounding of the Environment, Lake Arrowhead, California, 1-4 March 2015.

Roosjen, P.P.J.; Bartholomeus, H.M.; Clevers, J.G.P.W. (2014). Effects of soil moisture content on reflectance anisotropy-laboratory goniometer measurements and RPV model inversions. In: 4th International Symposium on Recent Advances in Quantitative Remote Sensing, Valencia, Spain, 22-26 September 2014; pp 230-236.

Roosjen, P.P.J.; Bartholomeus, H.M.; Clevers, J.G.P.W. (2013). A Laboratory geometer facility for measuring reflectance anisotropy in support of the Sentinel missions. In: ESA Living Planet Symposium, Edinburgh, Scotland, 9-13 September 2013.

Roosjen, P.P.J.; Bartholomeus, H.M.; Clevers, J.G.P.W. (2013). Effects of soil moisture content on reflectance anisotropy. In: 4th Advanced Training Course in Land Remote Sensing, Athens, Greece, 1-5 July 2013.

Bartholomeus, H.M.; Roosjen, P.P.J.; Clevers, J.G.P.W.; Suomalainen, J.M.; Mulder, V.L.; Kooistra, L. (2013). Estimation of soil clay content using multidirectional laboratory spectroscopy measurements. In: Proceedings of the 16th International Conference on Near Infrared Spectroscopy, La Grande-Motte, France, 2-7 June 2013; pp 187-194. 
Bartholomeus, H.M.; Roosjen, P.P.J.; Clevers, J.G.P.W.; Suomalainen, J.M.; Kooistra, L.K. (2013). Estimation of soil clay content using multidirectional laboratory spectroscopy measurements. In: 8th EARSeL Imaging Spectroscopy Workshop, Nantes, France, 8-10 April 2013. 


\section{Short biography}

Peter Roosjen was born on 23 January 1986 in Wageningen, the Netherlands. After completing high school in Wageningen, Peter studied Industrial Design at the University of Twente from 2004 to 2006, after which he made a switch to Landscape Architecture and Spatial Planning at Wageningen University \& Research, where he obtained his Bachelor's degree in 2011. During this program, Peter developed an interest in geo-informatics and remote sensing and therefore, he started the Geo-Information Science master at the same university. During the master program, Peter did

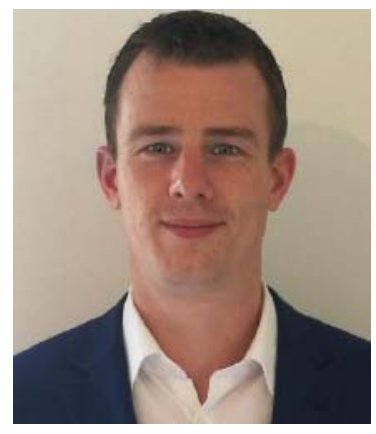
a research internship at the Remote Sensing Laboratories at the Department of Geography at the University of Zurich, where he studied different methods for topographic and BRDF correction of airborne data. For his master's thesis, Peter studied the effects of leaf orientation, canopy cover, and illumination geometry on the reflectance anisotropy of vegetated surfaces. The experimental work for his thesis was performed using a robotic goniometer and resulted in a peer reviewed publication. Shortly after obtaining his master's degree, Peter was hired as a PhD candidate at the Laboratory of Geo-Information Science and Remote at Wageningen University \& Research where he continued studying reflectance anisotropy using both goniometers and unmanned aerial vehicles, mainly exploring the information content of the anisotropy signal. His research resulted in this thesis and in several peer reviewed articles. Peter is currently working as a remote sensing consultant at Milan Innovincy and as a postdoctoral researcher at Laboratory of GeoInformation Science and Remote at Wageningen University \& Research. 


\section{PE\&RC Training and Education Statement}

With the training and education activities listed below the $\mathrm{PhD}$ candidate has complied with the requirements set by the C.T. de Wit Graduate School for Production Ecology and Resource Conservation (PE\&RC) which comprises of a minimum total of 32 ECTS $(=22$ weeks of activities).

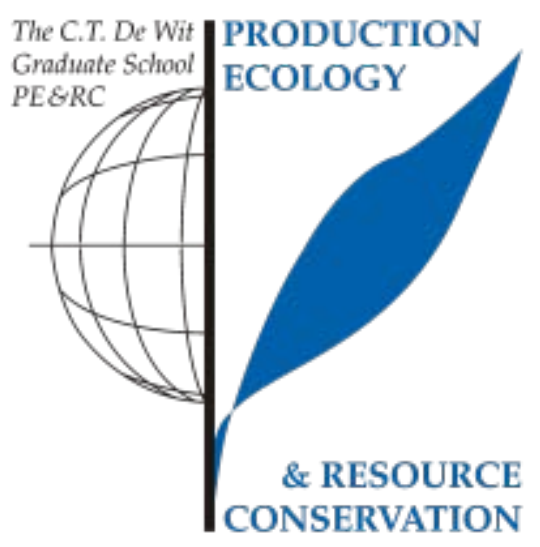

\section{Review of literature (6 ECTS)}

- Improving vegetation parameter retrieval using surface anisotropy modelling

\section{Post-graduate courses (4.4 ECTS)}

- Advanced geomatics in modern forestry; EARSeL (2016)

- Geostatistics; PE\&RC (2015)

- 4th Advanced training course in land remote sensing; ESA (2013)

Deficiency, refresh, brush-up courses (1.5 ECTS)

- Basic statistics; PE\&RC (2014)

\section{Competence strengthening / skills courses (3.6 ECTS)}

- Competence assessment; WGS (2015)

- Scientific writing; Wageningen into Languages (2014)

- Project and time management; WGS (2014) 
PE\&RC Annual meetings, seminars and the PE\&RC weekend (1.2 ECTS)

- PE\&RC Day (2014)

- PE\&RC First years weekend (2013)

Discussion groups / local seminars / other scientific meetings (9 ECTS)

- GreenVision (2014-2015)

- Remote Sensing Thematic Group Meeting (2013-2016)

International symposia, workshops and conferences (13.5 ECTS)

- Living Planet Symposium (2016)

- 9th EARSeL Imaging Spectroscopy Workshop (2015)

- Hyperspectral Imaging and Sounding of Environment (2015)

- 4th International Symposium on Recent Advances in Quantitative Remote Sensing (2014)

- $\quad$ Living Planet Symposium (2013)

- 8th EARSeL Imaging Spectroscopy Workshop (2013)

Lecturing / supervision of practical's / tutorials (2.4 ECTS)

- Advanced Earth Observations (2014-2015) 

The research described in this thesis was funded by a grant from the User Support Programme Space Research (GO/12-15) in The Netherlands.

Cover design by Peter P. J. Roosjen 


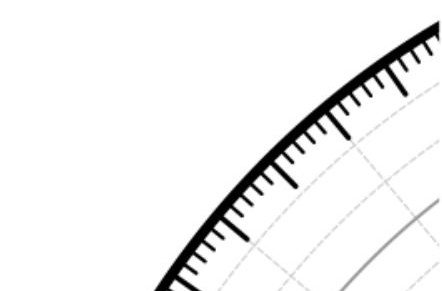

$E$ 